\title{
1 Unique structure and positive selection promote 2 the rapid divergence of Drosophila $Y$ 3 chromosomes
}

4 Ching-Ho Chang ${ }^{1 \# *}$, Lauren E. Gregory ${ }^{1}$, Kathleen E. Gordon ${ }^{2 \wedge}$, Colin D. Meiklejohn ${ }^{2}$

5 and Amanda M. Larracuente ${ }^{1 *}$

6 Affiliations:

$7 \quad{ }^{1}$ Department of Biology, University of Rochester, Rochester, NY 14627

$8 \quad{ }^{2}$ School of Biological Sciences, University of Nebraska-Lincoln, NE 68502

9 \#Current address: Division of Basic Sciences, Fred Hutchinson Cancer Research Center,

10 Seattle, WA 98109

$11{ }^{\wedge}$ Current address: Department of Molecular Biology and Genetics, Field of Genetics,

12 Genomics and Development, Cornell University, Ithaca, NY, 14853

15 *correspondence to cchang2@fredhutch.org, alarracu@ur.rochester.edu 


\section{Abstract}

22 Y chromosomes across diverse species convergently evolve a gene-poor,

23 heterochromatic organization enriched for duplicated genes, LTR retrotransposable

24 elements, and satellite DNA. Sexual antagonism and a loss of recombination play major

25 roles in the degeneration of young $\mathrm{Y}$ chromosomes. However, the processes shaping

26 the evolution of mature, already degenerated $\mathrm{Y}$ chromosomes are less well-understood.

27 Because $\mathrm{Y}$ chromosomes evolve rapidly, comparisons between closely related species

28 are particularly useful. We generated de novo long read assemblies complemented with

29 cytological validation to reveal Y chromosome organization in three closely related

30 species of the Drosophila simulans complex, which diverged only 250,000 years ago

31 and share $>98 \%$ sequence identity. We find these $Y$ chromosomes are divergent in their

32 organization and repetitive DNA composition and discover new $Y$-linked gene families

33 whose evolution is driven by both positive selection and gene conversion. These $Y$

34 chromosomes are also enriched for large deletions, suggesting that the repair of

35 double-strand breaks on $\mathrm{Y}$ chromosomes may be biased toward microhomology-

36 mediated end joining over canonical non-homologous end-joining. We propose that this

37 repair mechanism generally contributes to the convergent evolution of $Y$ chromosome 38 organization. 


\section{Introduction}

41 Most sex chromosomes evolved from a pair of homologous gene-rich autosomes that

42 acquired sex-determining factors and subsequently differentiated. Y chromosomes

43 gradually lose most of their genes, while their $\mathrm{X}$ chromosome counterparts tend to retain

44 the original autosomal complement of genes. This $Y$ chromosome degeneration follows

45 a suppression of recombination [1], which limits the efficacy of natural selection, and

46 causes the accumulation of deleterious mutations through Muller's ratchet, background

47 selection, and hitchhiking effects [2-6]. As a consequence, many $Y$ chromosomes

48 present a seemingly hostile environment for genes, with their mutational burden, high

49 repeat content and abundant silent chromatin.

50 Genomic studies of $Y$ chromosome evolution focus primarily on young sex

51 chromosomes, addressing how the suppression of recombination promotes $Y$

52 chromosome degeneration at both the epigenetic and genetic levels [2, 7]. Although

53 sexually antagonistic selection is traditionally cited as the cause of recombination

54 suppression on the $\mathrm{Y}$ chromosome, direct evidence for its role is still lacking [8] and new

55 models propose that regulatory evolution is the initial trigger for recombination

56 suppression [9]. Sexually antagonistic selection may accelerate Y-linked gene evolution

57 to optimize male-specific functions. Indeed, Y-linked genes tend to have slightly higher

58 rates of protein evolution than their orthologs on other chromosomes [10, 11]. Higher

59 rates of $\mathrm{Y}$-linked gene evolution are driven by positive selection, relaxed selective

60 constraints and male-biased mutation patterns, with most $Y$-linked genes evolving under 
61 at least some functional constraint [11]. Although there is evidence suggesting that

62 some $\mathrm{Y}$ chromosomes have experienced recent selective sweeps [12, 13], the relative

63 importance of positive selection for $Y$ chromosome evolution remains unclear.

$64 \mathrm{Y}$ chromosomes harbor extensive structural divergence between species, in part

65 through the acquisition of genes from other genomic regions [14-21]. However, the

66 functions of most $Y$-linked genes are unknown [18, 21-23]. Some $Y$-linked genes are

67 duplicated and, in extreme cases, amplified into so-called ampliconic genes-gene

68 families with tens to hundreds of highly similar sequences. Y chromosomes of both

69 Drosophila and mammals have independently acquired and amplified gene families,

70 which turnover rapidly between closely related species [14, 17, 20, 24-26]. Following Y-

71 linked gene amplification, gene conversion between gene copies may enhance the

72 efficacy of selection on Y-linked genes in the absence of crossing over [15, 27].

73 Detailed analyses of old $Y$ chromosomes have been restricted to a few species with

74 reference-quality assemblies, e.g., mouse and human. The challenges of cloning and

75 assembling repeat-rich regions of the genome have stymied progress towards a

76 complete understanding of $Y$ chromosome evolution [28-30]. Recent advances in long-

77 read sequencing make it feasible to assemble large parts of $Y$ chromosomes [19, 21,

7822,31 ] enabling comparative studies of a majority of $Y$-linked sequences in closely

79 related species.

80 Drosophila melanogaster and three related species in the $D$. simulans clade are ideally

81 suited to study $\mathrm{Y}$ chromosome evolution. These $\mathrm{Y}$ chromosomes are functionally 
82 divergent, contribute to hybrid sterility [32-35], and at least four X-linked meiotic drive

83 systems likely shape $\mathrm{Y}$ chromosome evolution in these species [36-43]. Previous

84 genetic and transcriptomic studies suggest that $Y$ chromosome variation can impact

85 male fitness and gene regulation [44-51]. Since there is minimal nucleotide variation

86 and divergence in Y-linked protein-coding sequences within and between these

87 Drosophila species [11, 12, 40], structural variation may be responsible for the majority

88 of these effects. For example, $20-40 \%$ of D. melanogaster $Y$-linked regulatory variation

89 (YRV) comes from differences in ribosomal DNA (rDNA) copy numbers [52, 53]. The

90 chromatin on Drosophila Y chromosomes has genome-wide effects on expression level

91 and chromatin states [54], but aside from the rDNA, the molecular basis of $Y$

92 chromosome divergence and variation in these species remains elusive.

93 To better understand $Y$ chromosome structure and evolution, we assembled the $Y$

94 chromosomes of the three species in the $D$. simulans clade and compared them to $D$.

95 melanogaster. We observe that the $Y$ chromosomes of the $D$. simulans clade species

96 have high duplication and gene conversion rates that, along with strong positive

97 selection, shaped the evolution of two new ampliconic protein-coding gene families. We

98 propose that, in addition to positive selection, sexual antagonism, and genetic conflict,

99 differences in the usage of DNA repair pathways may give rise to the unique patterns of

100 Y-linked mutations. Together these effects may drive the convergent evolution of $Y$

101 chromosome structure across taxa. 


\section{Results}

104 Improving the $Y$ chromosome assemblies using long-read assembly and

\section{fluorescence in situ hybridization (FISH)}

Long reads have enabled the assembly of many repetitive genome regions, but have had limited success in assembling $Y$ chromosomes [17, 19, 21, 22]. To improve $Y$ chromosome assemblies for comparative genomic analyses, we applied our heterochromatin-sensitive assembly pipeline [22] with long reads that we previously generated [55] to reassemble the $Y$ chromosome from the three species in the

111 Drosophila simulans clade. We also resequenced male genomes using PCR-free

112 Illumina libraries to polish these assemblies. Our heterochromatin-enriched methods

113 improve contiguity compared to previous $D$. simulans clade assemblies. We recovered

114 all known exons of the 11 canonical Y-linked genes conserved across the melanogaster 115 group, including 58 exons missed in previous assemblies (Table S1; [55]). Based on the

116 median male-to-female coverage [22], we assigned 13.7 to $18.9 \mathrm{Mb}$ of $\mathrm{Y}$-linked

117 sequences per species with $\mathrm{N} 50$ ranging from 0.6 to $1.2 \mathrm{Mb}$. The quality of these new

118 D. simulans clade Y assemblies are comparable to D. melanogaster (Table 1; [22]). We 119 evaluated our methods by comparing our assignments for every 10-kb window of 120 assembled sequences to its known chromosomal location. Our assignments have 96,

12198 , and 99\% sensitivity and 5, 0, and 3\% false-positive rates in $D$. mauritiana, $D$.

122 simulans, and $D$. sechellia, respectively (Table S2). We have lower confidence in our $D$.

123 mauritiana assignments, because the male and female Illumina reads are from different 124 library construction methods. Therefore, we applied an additional criterion only in $D$.

125 mauritiana based on the female-to-male total mapped reads ratio $(<0.1)$, which reduces 
126 the false-positive rate from 13 to $5 \%$ in regions with known chromosomal location (Table

127 S2; Fig S1). Based on these chromosome assignments, we find $40-44 \%$ lower PacBio

128 coverage on $\mathrm{Y}$ than $\mathrm{X}$ chromosomes in all three species (Fig S2).

Table 1. Contiguity statistics for heterochromatin-enriched assemblies

\begin{tabular}{lrrr}
\hline $\begin{array}{l}\text { Y chromosome } \\
\text { assembly }\end{array}$ & \# of contigs & Total length & Contigs N50 \\
\hline D. melanogaster & 80 & $14,578,684$ & 416,887 \\
D. mauritiana $^{\mathrm{b}}$ & 55 & $17,880,069$ & $1,628,994$ \\
D. simulans $^{\mathrm{b}}$ & 38 & $13,717,056$ & $1,031,383$ \\
D. sechellia $^{\mathrm{b}}$ & 63 & $14,899,148$ & 555,130
\end{tabular}

${ }^{\mathrm{a} C h a n g}$ and Larracuente 2019

131 bThis paper

133 The cytological organization of the $D$. simulans clade $Y$ chromosomes is not welldescribed [56-58]. Therefore, we generated new physical maps of the $Y$ chromosomes by combining our assemblies with cytological data. We performed FISH on mitotic

136 chromosomes using probes for 12 Y-linked sequences (Fig 1 and S3-4; Table S3) to 137 determine $\mathrm{Y}$ chromosome organization at the cytological level. We also determined the

138 location of the centromeres using immunostaining with a Cenp-C antibody (Fig S4;

139 [59]). These cytological data permit us to 1) validate our assemblies, and 2) infer the

140 overall organization of the $\mathrm{Y}$ chromosome by orienting our scaffolds on cytological

141 maps. Of the $11 \mathrm{Y}$-linked genes, we successfully ordered 10, 11, and 7 genes on the

142 cytological bands of D. simulans, D. mauritiana and D. sechellia, respectively (Fig 1 and

143 S3). We find evidence for extensive $Y$ chromosomal structural rearrangements,

144 including changes in satellite distribution, gene order, and centromere position. These 
rearrangements are dramatic even among the $D$. simulans clade species, which

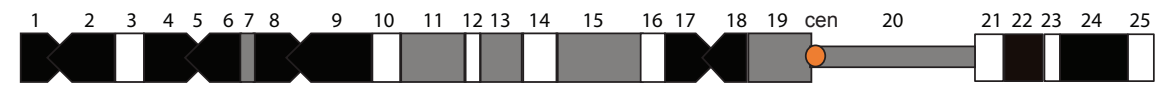

D. mauritiana
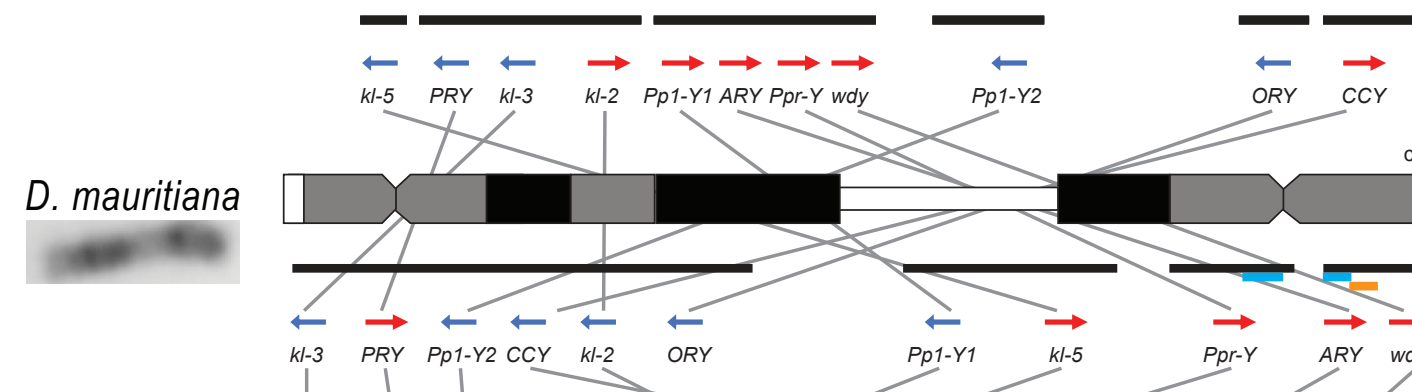

D. simulans

Fig 1. Y chromosome organization in $D$. melanogaster and the three $D$. simulans clade species. Schematics of the cytogenetic maps note the locations of Y-linked genes in $D$. melanogaster and $D$. simulans clade species. The bars show the relative placement of the scaffolds on the cytological bands based on FISH results. The solid black and dotted bars represent the scaffolds with known and unknown orientation information, respectively. The light blue and orange bars represent two new $\mathrm{Y}$-linked gene families, Lhk and CK2ßtes-Y in the $D$. simulans clade, respectively. The arrows indicate the orientation of the genes (blue- minus strand; red- plus strand). 


\section{Y-linked sequence and copy number divergence across three species}

163 Although the $D$. simulans clade species diverged only recently, Y chromosome

164 introgression between pairs of species disrupts male fertility and influences patterns of

165 genome-wide gene expression [32, 34]. One candidate locus that may contribute to

166 functional divergence and possibly hybrid lethality is the Y-linked rDNA [52, 60]. Y-

167 linked rDNA, specifically 28S rDNA, have been lost in $D$. simulans and $D$. sechellia, but

168 not in D. mauritiana [57, 61, 62]. However, the intergenic spacer (IGS) repeats between

169 rDNA genes, which are responsible for X-Y pairing in D. melanogaster males [63], are

170 retained on both sex chromosomes in all three species [57, 61, 62]. Consistent with

171 previous cytological studies $[57,61,62]$, we find that $D$. simulans and $D$. sechellia lost

172 most Y-linked $18 S$ and 28 S rDNA sequences (Fig S5). Our assemblies indicate that,

173 despite this loss of the rRNA coding sequences, all three species still retain IGS

174 repeats. However, we and others do not detect Y-linked IGS repeats at the cytological

175 level in D. sechellia (Fig S3-4; [57, 61, 62]), suggesting that their abundance is below

176 the level of detection by FISH in this species.

177 Structural variation at $\mathrm{Y}$-linked genes may also contribute to functional variation and

178 divergence in the $D$. simulans clade. Previous studies reported many duplications of

179 canonical Y-linked genes in D. simulans $[40,55,64]$. We find that all three species have

180 at least one intact copy of the 11 canonical Y-linked genes, but there is also extensive

181 copy number variation in Y-linked exons across these species (Figure 2 and S6-7,

182 Table S1; [65]). Using Illumina reads, we confirm the copy number variation in our

183 assemblies, and further reveal some $\mathrm{Y}$-linked duplicated exons, particularly in $k l-3$, wdy

184 and Ppr-Y, that are not assembled in D. sechellia (Fig S6). Some duplicates may be 
185 functional because they are expressed and have complete open reading frames, (e.g., ARY, Ppr-Y1 and Ppr-Y2). The D. simulans Y chromosome has four complete copies of $A R Y$, all of which show similar expression levels from RNA-seq data (Figure $2 \mathrm{~B}$ and Table S4), but two copies have inverted exons 1 and 2. D. sechellia also contains at least five duplicated copies of $A R Y$, some of which also have the inverted exons 1 and 2 , but the absence of RNA-seq data from testes of this species prevents inferences regarding whether all copies of $A R Y$ are expressed. However, most duplications include only a subset of exons, and in many cases, the duplicated exons are located on the periphery of the presumed functional gene copy (Figure 2B and S7, Table S4). For example, both $D$. simulans and $D$. mauritiana have multiple copies of exons 8-12 located at the 3 ' end of $k l-2$ (Figure 2B). In D. simulans, most of these extra exons have low to no expression, while in D. mauritiana, there appears to be a substantial expression from many of the duplicated terminal exons, as well as an internal duplication of exon 5. It is unclear what effects these duplicated exons have on the protein sequences of these fertility-essential genes.

200 All exon-intron junctions are conserved within full-length copies of the canonical $Y$ 201 linked genes, yet intron lengths vary between these species (Fig 3). The length of 202 longer introns (>100 bp in any species) is more dynamic than that of short introns (Fig 3; 203 Table S5). The dramatic size differences in most introns cannot be attributed to a single 204 deletion or duplication (see an example of ORY in Fig S8). Some Y-linked genes 205 contain mega-base sized introns (i.e., mega-introns) whose transcription manifests as 206 cytologically visible lampbrush-like loops (Y-loops) in primary spermatocytes [66, 67]. 207 While Y-loops are found across the Drosophila genus [68, 69], their potential functions 
208 are unknown [70-74] and the genes/introns that produce Y-loops differs among species

209 [75] (Supplemental text). D. melanogaster has three Y-loops transcribed from introns of

210 ORY (ks-1 in previous literature), kl-3, and kl-5 [66]. Based on cytological evidence, $D$.

211 simulans has three Y-loops, whereas $D$. mauritiana and $D$. sechellia only have two [69].

212 Of all potential loop-producing introns, we find that only the $k l-3$ mega-intron is

213 conserved in all four species and has the same intron structure and sequences (i.e.,

214 (AATAT) $)_{n}$ repeats). While both $k l-5$ and ORY produce Y-loops with (AAGAC) $)_{\mathrm{n}}$ repeats in

215 D. melanogaster, (AAGAC) $)_{\mathrm{n}}$ is missing from the genomes of the $D$. simulans clade

216 species. This observation is supported by our assemblies, the Illumina raw reads (Table

217 S3), and published FISH results [76]. In the D. simulans clade, the ORY introns do not

218 carry any long tandem repeats. However, $k l-5$ has introns with (AATAT)n repeats that

219 may form a Y-loop in the $D$. simulans clade species. These data suggest that, while

220 mega-introns and Y-loops may be conserved features of spermatogenesis in

221 Drosophila, they turn over at both the sequence and gene levels over short periods of

222 evolutionary time (i.e., 2 My between $D$. melanogaster and the $D$. simulans clade). 
A

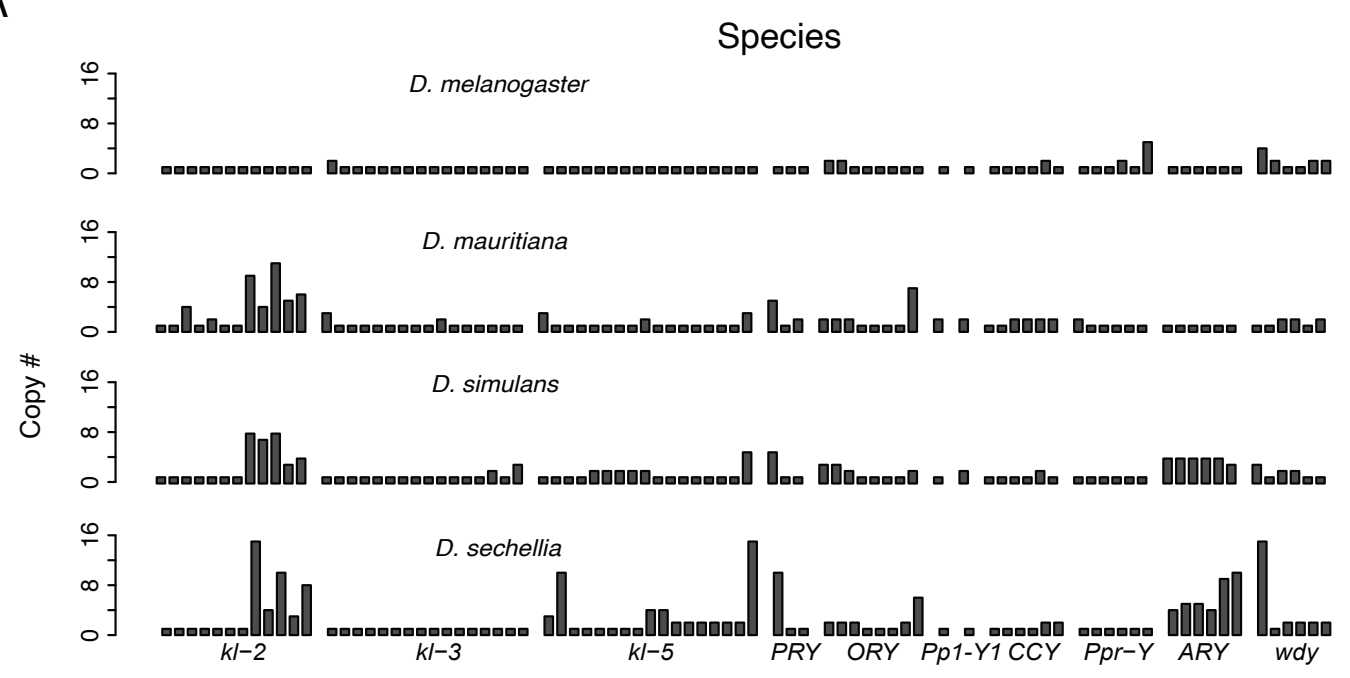

B

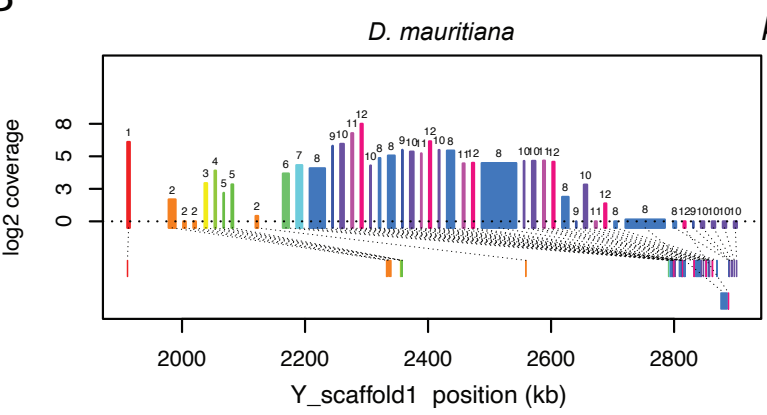

$k l-2$

D. simulans

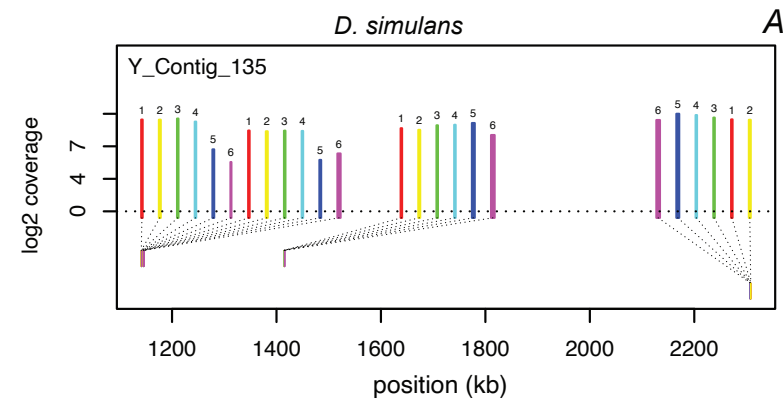

ARY
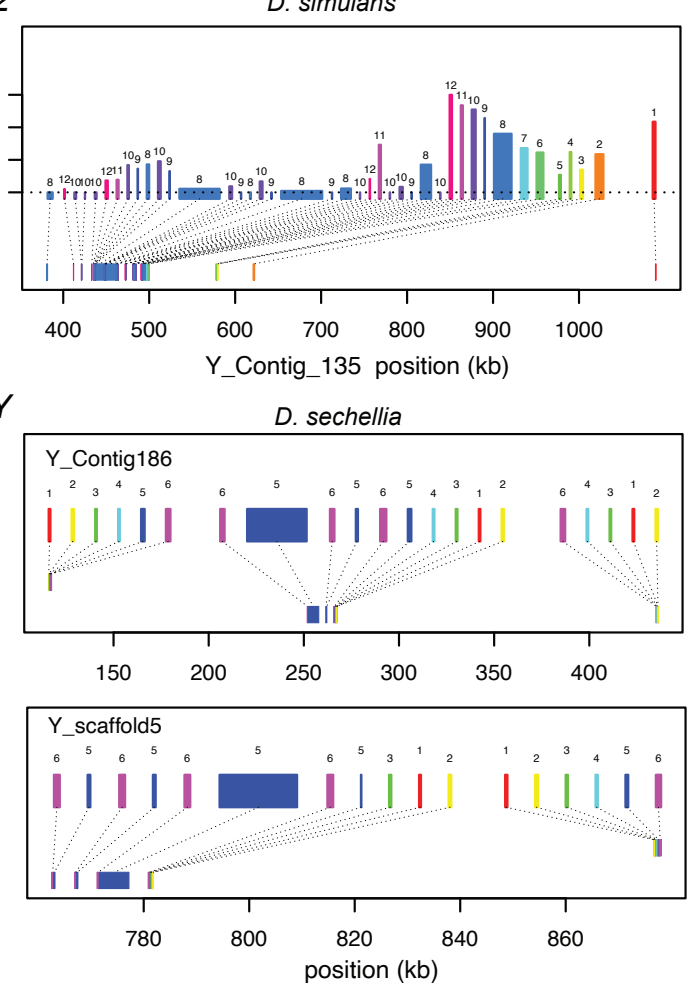

Fig 2. Duplication of canonical Y-linked exons. A) Exon copy number is highly variable across the three $D$. simulans clade species and generally greater than in $D$. melanogaster. B) Gene structure of $k l-2$ and $A R Y$ inferred from assemblies and RNAseq data. Upper bars indicate exons that are colored and numbered, with their height showing average read depth from sequenced testes RNA $(D$. simulans and $D$. mauritiana only). Lower bars indicate exon positions on the assembly and position on the $\mathrm{Y}$-axis indicates coding strand. 
233 Consistent with previous studies [18, 55], we identify high rates of gene duplication to

234 the $D$. simulans clade $\mathrm{Y}$ chromosome from other chromosomes. We find 49

235 independent duplications to the $\mathrm{Y}$ chromosome in our heterochromatin-enriched

236 assemblies (Fig 4; Table S6), including eight newly discovered duplications [18, 55].

237 Twenty-eight duplications are DNA-based, 13 are RNA-based, and the rest are

238 unknown due to the limited sequence information (Table S6). The rate of transposition

239 to the $\mathrm{Y}$ chromosome is about 3-4 times higher in the $D$. simulans clade compared to $D$.

240 melanogaster [22]. We also infer that 17 duplicated genes were independently deleted

241 from $D$. simulans clade $Y$ chromosomes. Based on transcriptomes from $D$. simulans

242 and $D$. mauritiana testes, we suspect that more than half of the duplicated genes are

243 likely pseudogenes that either show no expression in testes (<3 TPM) or lack open

244 reading frames (<100 amino acids; Table S6). We also detect intrachromosomal

245 duplications of these Y-linked pseudogenes (Table S6), suggesting a high duplication

246 rate within these $Y$ chromosomes.

247

248

249

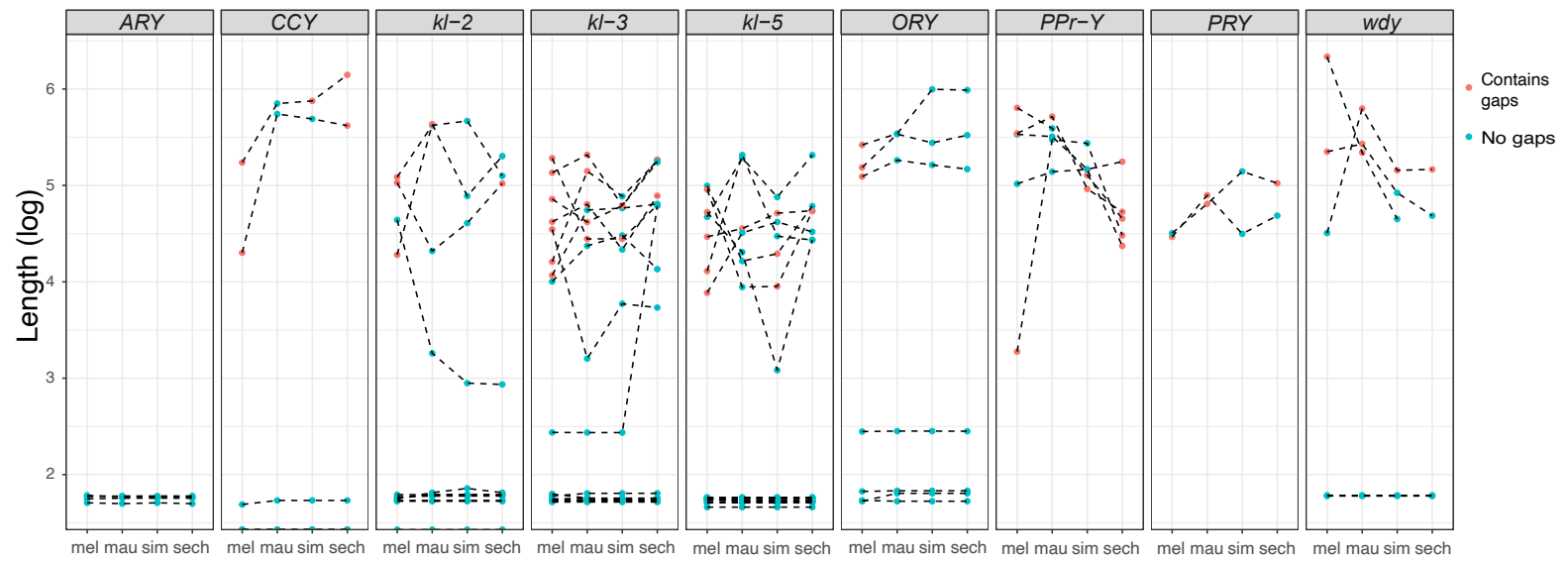

Fig 3. Evolution of intron lengths in canonical $Y$-linked genes. The intron length in canonical Y-linked genes is different between $D$. melanogaster and the three $D$. 
simulans clade species. Orthologous introns are connected by dotted lines. Completely assembled introns are in blue and introns with gaps in the assembly are in red, and are 252 therefore minimum intron lengths.

\section{\# of Gained \# of Lost}

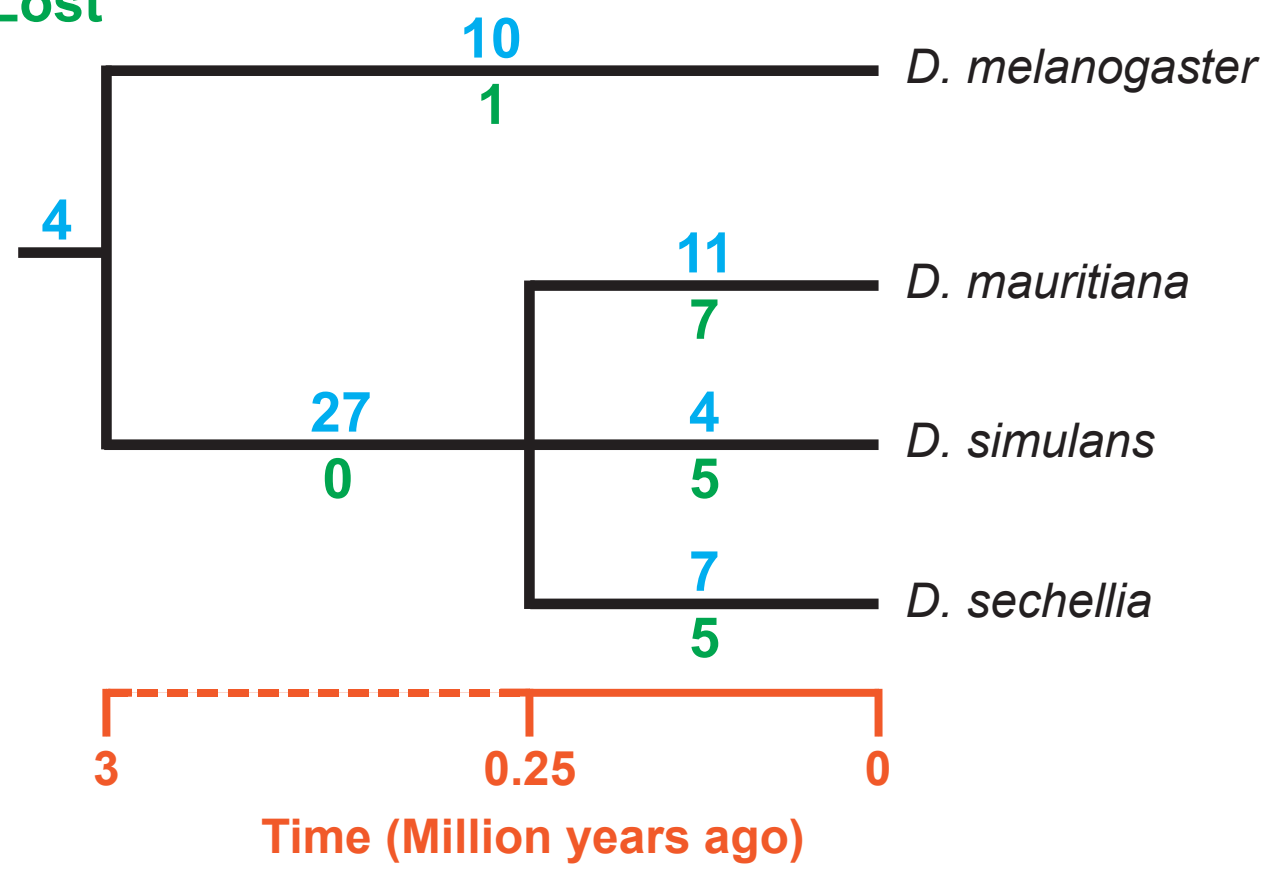
the evolutionary histories of new $Y$-linked duplications. The blue and green numbers represent the number of independent duplications and deletions observed in each branch, respectively. The deletion events that happened in the ancestor of these four species cannot be inferred without a $Y$ chromosome assembly in the outgroup.

Most new Y-linked genes in $D$. melanogaster and the $D$. simulans clade have presumed consistent with other Drosophila species [17, 77]. Y-linked duplicates of genes with

264 these functions may be selectively beneficial, but a duplication bias could also contribute to this enrichment, as genes expressed in the testes may be more likely to

266 duplicate to the $Y$ chromosome due to its open chromatin structure and transcriptional 267 activity during spermatogenesis [78-80]. 


\section{The evolution of new Y-linked gene families}

269 Ampliconic gene families are found on $\mathrm{Y}$ chromosomes in multiple Drosophila species

270 [24]. We discovered two new gene families that have undergone extensive amplification

271 on $D$. simulans clade $Y$ chromosomes. Both families appear to encode functional

272 protein-coding genes with complete open reading frames and high expression in

273 mRNA-seq data (Table S8), and have 36-146 copies in each species' Y chromosome.

274 We also confirm that $>90 \%$ of the variants in our assembled Y-linked gene families are

275 represented in Illumina DNA-seq data (Supplemental text).

276 The first amplified Y-linked gene family, SR Protein Kinase (SRPK), is derived from an

277 autosome-to-Y duplication of the sequence encoding the testis-specific isoform of the

278 gene SR Protein Kinase (SRPK). After the duplication of SRPK to the Y chromosome,

279 the ancestral autosomal copy subsequently lost its testis-specific exon via a deletion

280 (Figure 5A). The movement of the male-specific isoform inspired us to name the $\mathrm{Y}$ -

281 linked SRPK gene family Lo-han-kha (Lhk), which is the Taiwanese term for the male

282 vagabonds that moved from mainland China to Taiwan during the Qing dynasty. In $D$.

283 melanogaster, SRPK is essential for both male and female reproduction [81],

284 suggesting the hypothesis that the relocation of the testis-specific isoform to the $D$.

285 simulans clade $\mathrm{Y}$ chromosomes may have relieved intralocus sexual antagonism over

286 these two functions. Our phylogenetic analysis identified two subfamilies of $L h k$ that we

287 designate Lhk-1 and Lhk-2 (Figure 5B). Both subfamilies are shared by all D. simulans

288 clade species and show a 5.5\% protein divergence between species. The two

289 subfamilies are found in different locations in our $Y$ chromosome assemblies; consistent 
290 with this observation, we detect two to three Lhk foci on Y chromosomes in the $D$.

291 simulans clade using FISH (Figure 5B and 5D and Fig S3 and S4).

292 The second amplified gene family comprises both X-linked and Y-linked duplicates of

293 the Ss/ gene located on chromosome 2R; it is unclear whether the X- or Y-linked copies

294 originated first. The X-linked copies are known as CK2ßtes-like in D. simulans [82]. The

295 Y-linked copies are also found in D. melanogaster, but are degenerated and have little

296 or no expression [22, 83], leading to their designation as pseudogenes. In the $D$.

297 simulans clade species, however, the Y-linked paralogs have high levels of expression

298 (> 50 TPM in testes, Table S8) and complete open reading frames, so we refer to this

299 gene family as CK2ßtes-Y. Both CK2ßtes-like (4-9 copies) and CK2ßtes-Y (36-123

300 copies based on the assemblies) are amplified on the $X$ and $Y$ chromosome in the $D$.

301 simulans clade relative to $D$. melanogaster (Table S8) [82]. The Y-linked copies in $D$.

302 melanogaster, Su(Ste), are known to be a source of piRNAs [84]. We did not detect any testis piRNAs from either gene family in two small RNA-seq datasets (SRR7410589 and SRR7410590), however, we do find some short $(<23-n t)$ reads $(0.003-0.005 \%$ of total

305 mapped reads) mapped to these gene families (Table S9).

306 We inferred gene conversion rates and the strength of selection on these Y-linked gene

307 families using phylogenetic analyses on coding sequences. We estimated the gene

308 conversion rate in $D$. simulans clade $\mathrm{Y}$-linked gene families based on four-gamete tests

309 and gene similarity $[15,22,85,86]$. In general, $D$. simulans clade species show similar

310 gene conversion rates (on the order of $10^{-4}$ to $10^{-6}$ ) in both of these families compared to

311 our previous estimates in D. melanogaster (Table S10; [22]). These higher gene 
312 conversion rates compared to the other chromosomes might be a shared feature of $Y$

313 chromosomes across taxa [15].

314 To estimate rates of molecular evolution, we conducted branch-model and branch-site-

315 model tests on the reconstructed ancestral sequences of Lhk-1, Lhk-2, CK2ßtes-Y, and

316 two CK2ßtes-like using PAML (Fig 5B and 5C; [87]). We used reconstructed ancestral

317 sequences for our analyses to avoid sequencing errors in the assemblies, which appear

318 as singletons. We infer that after the divergence of $D$. simulans clade species, Lhk-1

319 evolved under purifying selection, whereas Lhk-2 evolved under positive selection (Fig

320 5B; Fig S9; Table S11). Using transcriptome data, we observe that highly expressed

321 Lhk-1 copies have fewer nonsynonymous mutations than lowly expressed copies in $D$.

322 simulans, consistent with purifying selection (Chi-square test's P=0.01; Fig S10 and

323 Table S12). Both Lhk gene families are expressed 2 to 7-fold higher than the ancestral

324 copy on $2 \mathrm{R}$ in the same species, and 1.9 to 64 -fold higher than their ortholog, SRPK-

$325 R C$, in D. melanogaster, suggesting that gene amplification may confer increased

326 expression. In both $D$. simulans and $D$. mauritiana, $L h k-1$ is shorter due to deletions

327 following its origin and has a higher expression level than Lhk-2. Both Lhk gene families

328 have higher copy numbers in $D$. simulans than $D$. mauritiana, which likely contributes to

329 their higher expression level in D. simulans (Table S8). For both Lhk-1 and Lhk-2,

330 copies from the same species are more similar than copies from other species-a

331 signal of concerted evolution [88]. 
A

Ancestral state (D. melanogaster, 2R)
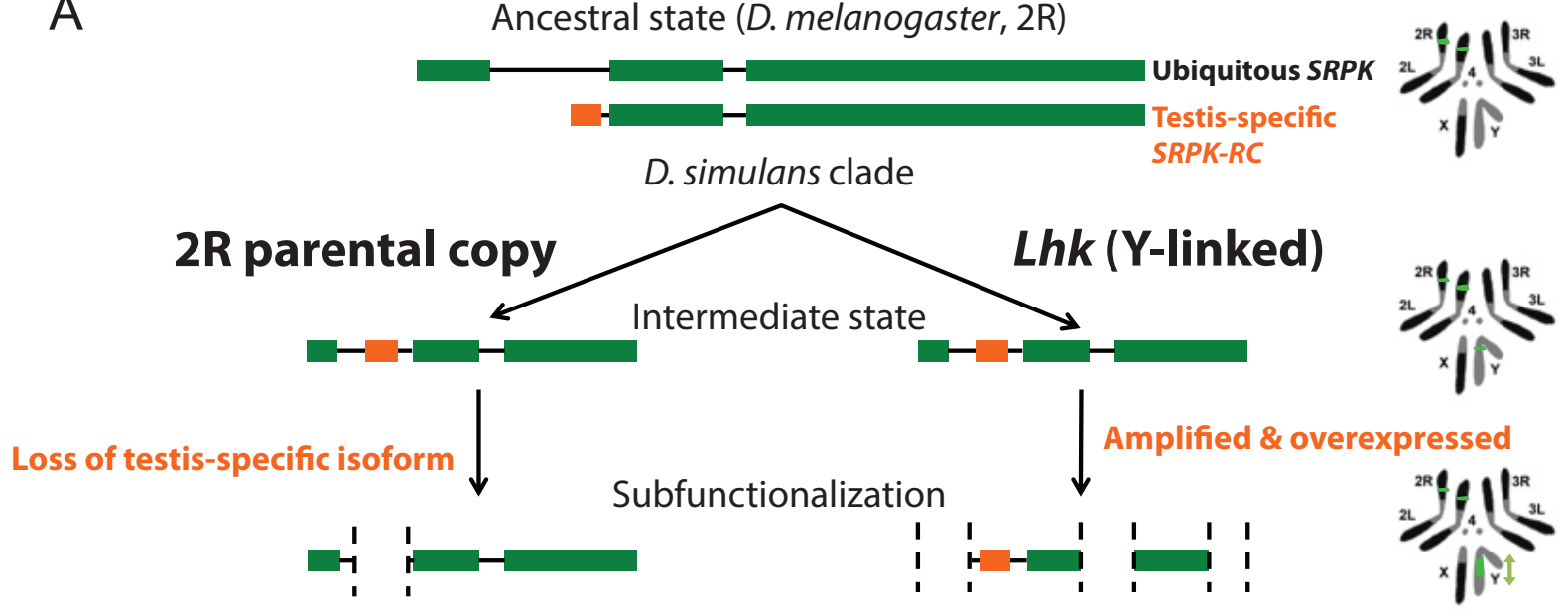

\section{B Lhk}

Species Location

- Dmel I $2 \mathrm{R}$

- Dmau - Y_cen

- Dsech $\Delta$ Y_other $_{-}$

- Dsim

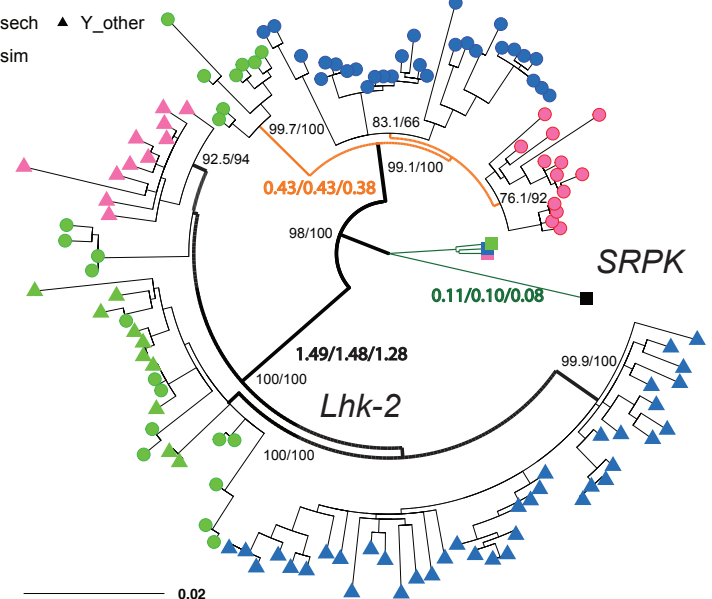

C CK2ßtes-Y

Location

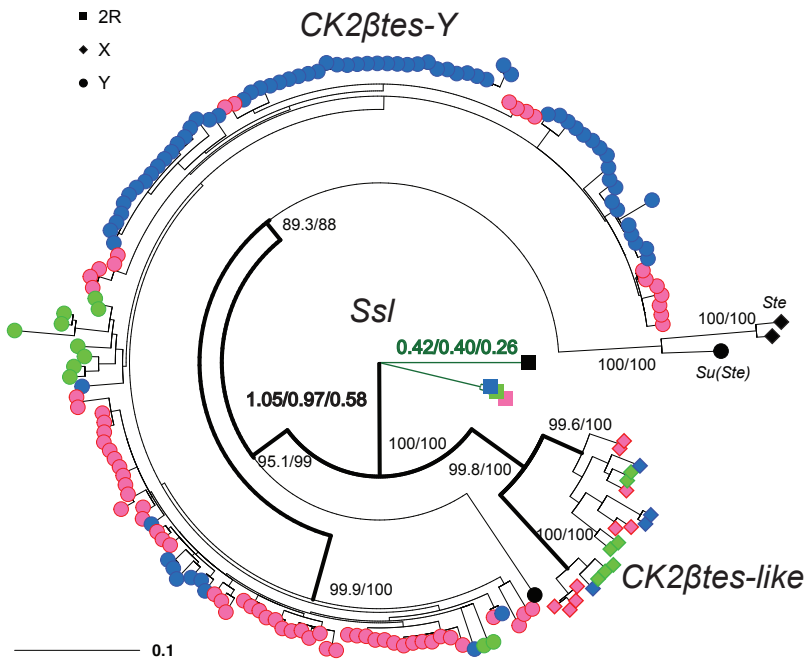

D

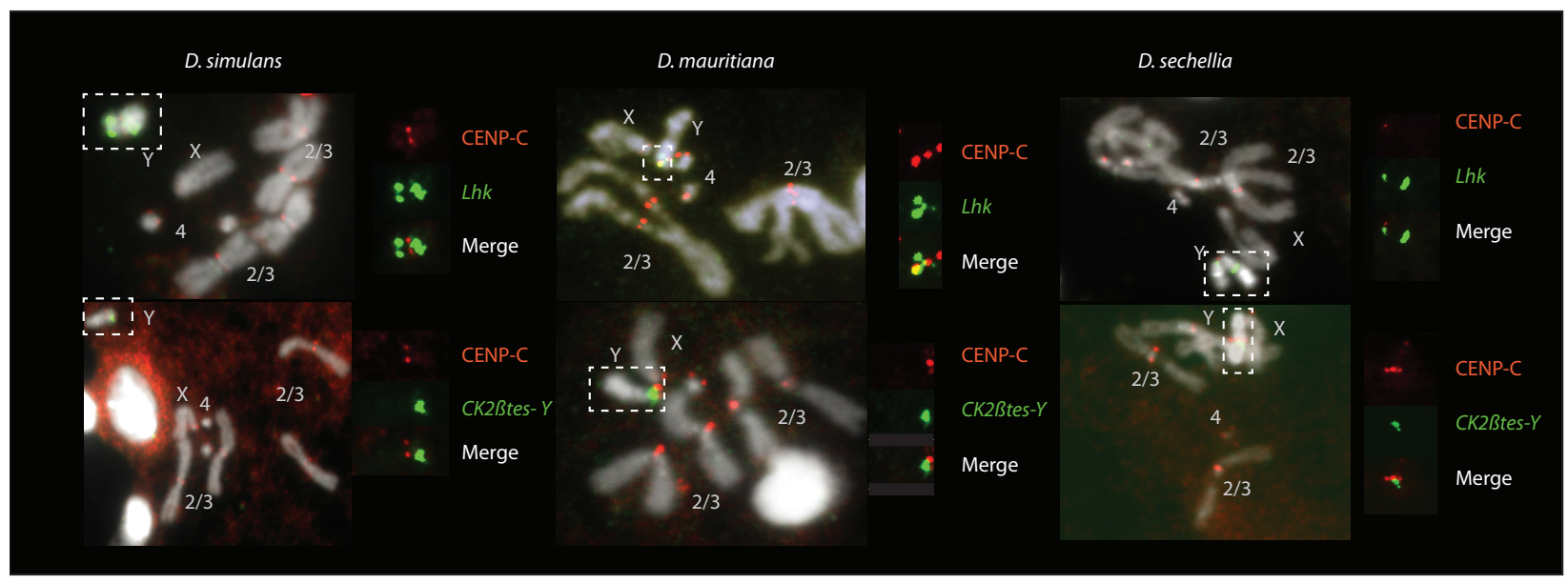


334 Fig 5. The rapid evolution and gene conversion of Y-linked ampliconic genes. A)

335 Schematic showing the inferred evolutionary history of SRPK-Y. SRPK duplicated to the 336 ancestral $Y$ chromosome in the $D$. simulans clade. The $Y$-linked copy (Lhk) retained an 337 exon with testis-specific expression, which was lost in the parental copy on $2 \mathrm{R}$. The $\mathrm{Y}$ 338 linked copy (Lhk) further duplicated and increased their expression in testes. B) The 339 inferred maximum likelihood phylogeny for Lhk. Node labels indicate SH-aLRT and 340 ultrafast bootstrap (e.g. 100/100) or rates of protein evolution from PAML with 341 CodonFreq $=0,1$, or 2 (e.g. 1.01/1.02/1.03) (Fig S9 and S11). Lhk shows evidence for 342 positive selection (branch tests and branch-site tests with $\omega>1$ ) after the duplication 343 from $2 \mathrm{R}(S R P K)$ to the $\mathrm{Y}$ chromosome in the $D$. simulans clade. One $L h k$ subfamily $344 \quad(L h k-1)$ is under recent purifying selection and is located close to the centromere, but 345 the other (Lhk-2) is rapidly evolving across the species of the $D$. simulans clade. $C)$ 346 Same as B but for CK2ßtes-Y. Both Y-linked CK2ßtes-Y and X-linked CK2ßtes-like also 347 show positive selection. All $\omega$ values shown are statistically significant (LRT tests, $348 \quad \mathrm{P} \leq 0.05$; Table S11 and S12). D) On the $\mathrm{Y}$ chromosomes, Lhk FISH signals are located 349 in 2-3 cytological locations. CK2ßtes- $Y$ signals are only located nearby centromeres in 350 the immunolabelling with fluorescent in situ hybridization (immunoFISH) experiments. 351 Based on our analysis of sequence information, we suggest that most Lhk-1 copies are 352 located close to CK2ßtes-Y and centromere. 
353 Table 2. PAML analyses reveal positive selection on Y-linked ampliconic gene families

\begin{tabular}{|c|c|c|c|c|c|c|c|c|c|c|c|c|c|}
\hline \multirow[b]{2}{*}{ Lhk } & \multicolumn{6}{|c|}{ Branch test with CodonFreq $=0$} & \multicolumn{7}{|c|}{ Branch-site test site class } \\
\hline & $\omega 1$ & $\omega 2$ & $\omega 3$ & L & $2 \Delta \operatorname{lnL}$ & LRT's P & $\omega 0$ & $\omega 1$ & $\omega 2 a$ & $\omega 2 b$ & $2 \Delta \operatorname{lnL}$ & LRT's P & Positively selected sites $(B E B>0.95)^{d}$ \\
\hline one $\omega$ & 0.17 & & & -3250.74 & & & & & & & & & \\
\hline two $w^{\mathrm{a}}$ & 0.11 & 1.05 & & -3218.26 & 64.94 & $7.71 \mathrm{E}-16$ & 0.01 & 1 & 4.87 & 4.87 & 13.04 & 3.05E-04 & I4, H11, V32, V75, N99, Y100, D193, D199 \\
\hline three $\omega^{c}$ & 0.11 & 1.49 & 0.43 & -3216.30 & 3.92 & 0.05 & & & & & & & \\
\hline \multicolumn{14}{|l|}{ CK2ßtes } \\
\hline one $\omega$ & 0.35 & & & -3295.01 & & & & & & & & & \\
\hline two $\omega^{\mathrm{b}}$ & 0.25 & 1.05 & & -3272.00 & 46.01 & $1.18 \mathrm{E}-11$ & 0.05 & 1 & 2.21 & 2.21 & 6.54 & 1.06E-02 & D33, T38, K44, K100, F101, K104, M152, M155 \\
\hline three $\omega^{c}$ & 0.20 & 0.42 & 1.05 & -3266.33 & 11.35 & 7.56E-04 & & & & & & & \\
\hline \multicolumn{14}{|c|}{ a Autosomal and Y lineage have protein evolution of $\omega 1$ and $\omega 2$, respectively. } \\
\hline \multicolumn{14}{|c|}{ b Autosomal and sex chromosomal ( $X$ and $Y$ ) have protein evolution of $\omega 1$ and $\omega 2$, respectively. } \\
\hline \multicolumn{14}{|c|}{ c See Figure 3C and 3D for the assignment of lineage. } \\
\hline
\end{tabular}


355 The ancestral Ss/ gene experienced a slightly increased rate of protein evolution after it

356 duplicated to the $X$ and $Y$ chromosomes ( $\omega=0.41$ vs. 0.23; $P=0.03$; Fig 5C; Fig S11;

357 Table S13). We find that both CK2ßtes-like and CK2ßtes-Y share strong signals of

358 positive selection, based on branch-model and branch-site-model tests ( $\mathrm{P}=8.8 \mathrm{E}-9$; Fig

359 5C; Fig S11; Table S13). In D. melanogaster, the overexpression of the CK2ßtes-like X-

360 linked homolog, Stellate, can drive in the male germline by killing Y-bearing sperm and

361 generating female-biased offspring [89-91]. We suspect that CK2ßtes-like and CK2ßtes-

$362 Y$ might have similar functions and may also have a history of conflict. Therefore, the

363 co-amplification of sex-linked genes and positive selection on their coding sequences

364 may be a consequence of an arms race between sex chromosome drivers.

365 Y chromosome evolution driven by specific mutation patterns

366 The specific DNA-repair mechanisms used on $Y$ chromosomes might contribute to their

367 high rates of intrachromosomal duplication and structural rearrangements. Because $Y$

368 chromosomes lack a homolog, they must repair double-strand breaks (DSBs) by non-

369 homologous end joining (NHEJ) or microhomology-mediated end joining (MMEJ), which

370 relies on short homology (usually $>2$ bp) to repair DSBs [92]. Compared to NHEJ,

371 MMEJ is more error-prone and can result in translocations and duplications [93].

372 Preferential use of MMEJ instead of NHEJ could contribute to the high duplication rate

373 and extensive genome rearrangements that we observed on $Y$ chromosomes. To infer

374 the mechanisms of DSB repair on $Y$ chromosomes, we counted indels between $Y$-linked

375 duplicates and their parent genes for a set of 17 putative pseudogenes—both NHEJ

376 and MMEJ can generate indels, but NHEJ usually produces smaller indels (1-3 bp)

377 compared to MMEJ (> 3 bp) $[93,94]$. We also cataloged short stretches of homology 
378 between each duplicate and its parent. To compare Y-linked patterns of DSB repair to 379 other regions of the genome, we measured the size of polymorphic indels in intergenic regions and pseudogenes on the autosomes and $X$ chromosomes from population data in D. melanogaster (DGRP [95]) and D. simulans [96]. To the extent that these indels do not experience selection, their sizes should reflect the mutation patterns on each chromosome. We observe proportionally more large deletions on $\mathrm{Y}$ chromosomes (25\%

384 of $Y$-linked indels are $\geq 10$-bp deletions; Table S14) compared to other chromosomes in

385 both $D$. melanogaster $(12.8 \%$ and $15.2 \%$ of indels are $\geq 10$-bp deletions in intergenic 386 regions and pseudogenes) and $D$. simulans ( $7.3 \%$ of indels are $\geq 10$-bp deletions in 387 intergenic regions; all pairwise chi-square's $\mathrm{P}<1 \mathrm{e}-6$; Fig 4A; Table S15). The pattern of excess large deletions is shared in the three $D$. simulans clade species $Y$ chromosomes, but is not obvious in D. melanogaster (Fig 6B). However, because all $D$. melanogaster $\mathrm{Y}$-linked indels in our analyses are from copies of a single pseudogene (CR43975), it is difficult to compare to the larger samples in the simulans clade species

392 (duplicates from 16 genes). The differences in deletion sizes between the $\mathrm{Y}$ and other chromosomes are unlikely to be driven by heterochromatin or the lack of recombination

394 -the non-recombining and heterochromatic dot chromosome has a deletion size profile 395 more similar to the other autosomes in $D$. simulans (10.9\% of indels are $\geq 10-b p$ deletions). These results suggest that $Y$ chromosomes may use MMEJ over NHEJ

397 compared to other chromosomes, particularly in the simulans clade species. We also 398 find that across the genome larger deletions (>7bp) share a similar length of 399 microhomologies for repairing DSBs-(39.5-57\% deletions have $\geq 2$ bp microhomology; 400 Chi-square test for microhomology length between $Y$ and other chromosomes, $P>0.24$; 
401 Table S14 and S15), consistent with most being a consequence of MMEJ-mediated

402 repair.

A

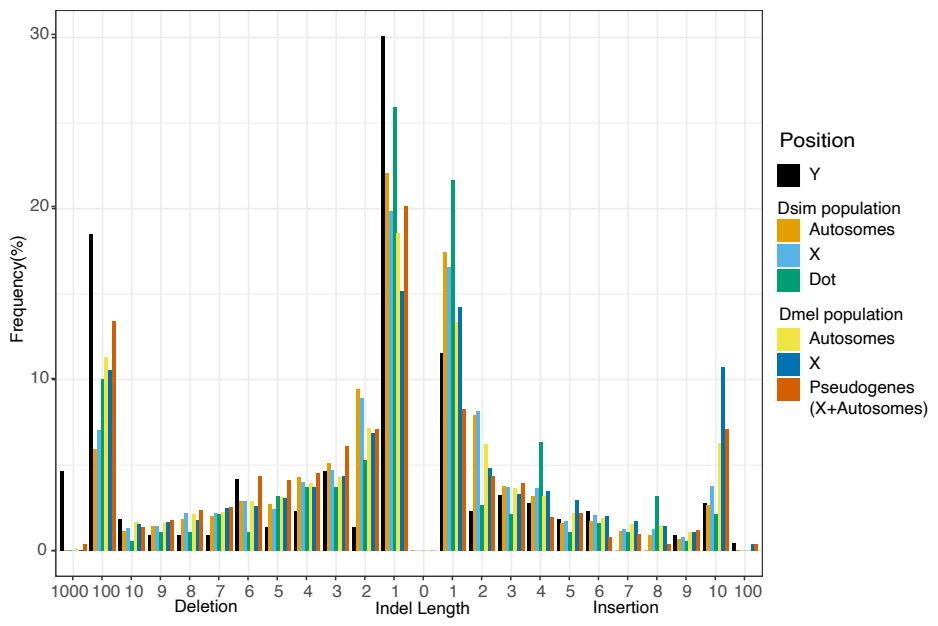

B Y chromosome only

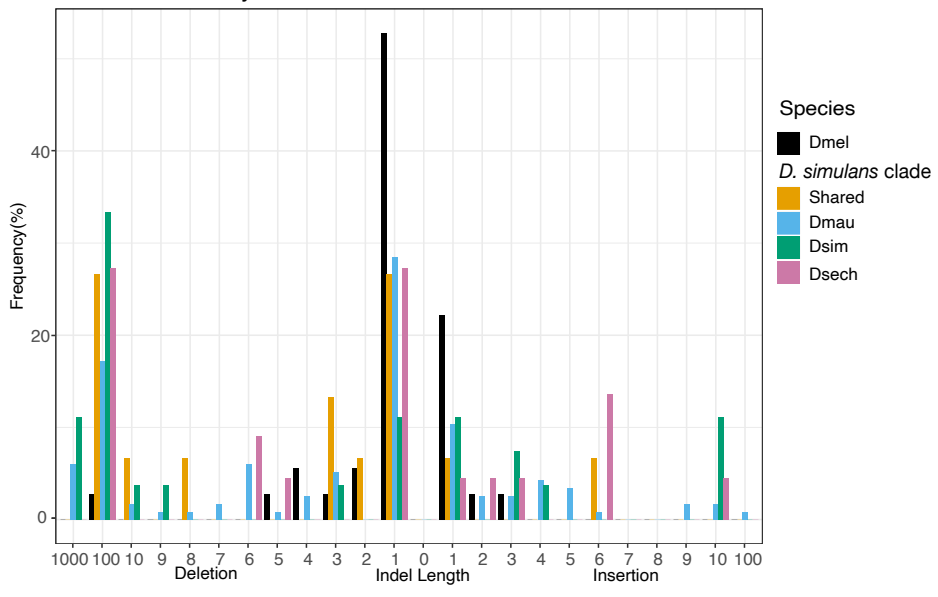

C

GAGAAGACCTCCAGCACCTGTTCGCACTGCTTGGCCACCTGGACCAGGT

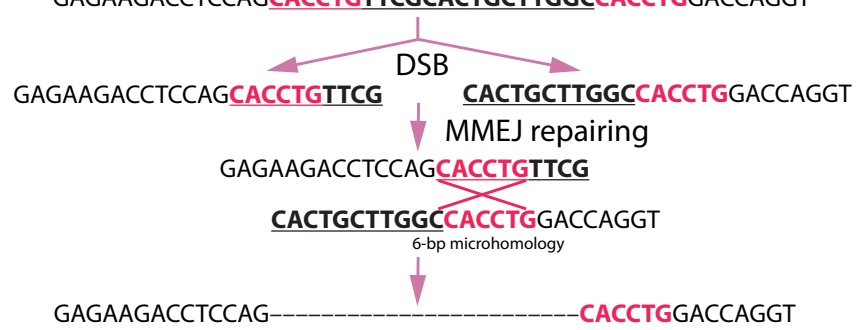

Fig 6. An excess of large deletions on $Y$ chromosomes, compared to population data suggests a preference for MMEJ. A) We compared the size of 216 indels on 17 recently duplicated $Y$-linked genes in $D$. melanogaster and the $D$. simulans clade species to the indels polymorphic in the $D$. melanogaster and $D$. simulans populations. 
408 For the indels in D. melanogaster and D. simulans populations, we separated them based on their location, including autosomes (excluding dot chromosomes), $\mathrm{X}$ chromosomes, and dot chromosomes. We excluded the D. melanogaster dot-linked indels due to the small sample size (12). B) We classify Y-linked indels by whether they are shared between species or specific in one species $\mathrm{C}$ ) The excess of large deletions (underlined) on the $\mathrm{Y}$ chromosomes is consistent with MMEJ between short regions of microhomology (red).

416 The satellite sequence composition of $Y$ chromosomes differs between species [76, 97, 417 98]. A high duplication rate may accelerate the birth and turnover of $Y$-linked satellite 418 sequences. We discovered five new Y-linked satellites in our assemblies and validated 419 their location using FISH (Fig S3-4 and Table S16). These satellites only span a few 420 kilobases of sequences (5,515 to $26,119 \mathrm{bp})$ and are homogenized. According to its

421 flanking sequence, one new satellite, (AAACAT) $)_{n}$, originated from a DM412B

422 transposable element, which has three tandem copies of AAACAT in its long terminal

423 repeats. The AAACAT repeats expanded to 764 copies on the $\mathrm{Y}$ chromosome

424 specifically in D. mauritiana. The other four novel satellites are flanked by transposons

425 (<50 bp) and may derive from non-repetitive sequences. The MMEJ pathway may

426 contribute to the birth of new repeats, as this mechanism is known to generate tandem

427 duplications via template-switching during repair [93]. Short tandem repeats can be

428 further amplified via saltatory replication or unequal crossing-over between sister

429 chromatids.

430 Consistent with findings in other species [19, 22], we find an enrichment of LTR

431 retrotransposons on the $D$. simulans clade $Y$ chromosomes relative to the rest of the

432 genome (Table S17). Interestingly, we find that the Y-linked LTR retrotransposons also

433 turn over between species (Fig S12 and Table S18). We find a positive correlation 
434 between the difference in Y-linked TE abundance between $D$. melanogaster and each

435 of the $D$. simulans clade species versus the rest of the genome (rho $=0.45-0.50 ;$ Fig

436 S13 and Table S18). This suggests that global changes in transposon activity could

437 explain the differences in Y-linked TEs abundance between species. However, the

438 correlations between species within the $D$. simulans clade are weaker $(r h o<0.23$; Fig

439 S13 and Table S18), consistent with the possibility that some TEs may shift their

440 insertion preference between chromosomes. To test this hypothesis, we estimated the

441 ages of LTR retrotransposons by their length. We find that the recent insertions of LTR

442 transposons are differently distributed across chromosomes between species (Fig S14),

443 suggesting that insertion preferences towards genomic regions may differ for some TEs.

444 For example, we detect many recent DIVER element insertions on the $\mathrm{Y}$ chromosome

445 in D. simulans, but not in D. sechellia (Fig S9).

\section{Discussion}

448 Despite their independent origins, the degenerated $Y$ chromosomes of mammals, fish,

449 and insects have convergently evolved structural features of gene acquisition and

450 amplification, accumulation of repetitive sequences, and gene conversion. Here we

451 consider the mutational processes that contribute to this structure and its consequences

452 for $\mathrm{Y}$ chromosome biology. Our assemblies revealed extensive $\mathrm{Y}$ chromosome

453 rearrangements between three very closely related Drosophila species (Figure 1).

454 These rearrangements may be the consequence of rejoining telomeres after DSBs, as

455 telomere-specific sequences are embedded in non-telomeric regions of Drosophila Y

456 chromosomes $[58,99,100]$. We propose that four pieces of evidence suggest DSBs on 
457 Y chromosomes may be preferentially repaired using the MMEJ pathway. First, Y-linked

458 sequences are absent from the $\mathrm{X}$ chromosome, precluding repair of DSBs by

459 homologous recombination in meiosis. Second, NHEJ on Y chromosomes may be

460 limited because the Ku complex, which is required for NHEJ [94], is excluded from

461 HP1a-rich regions of chromosomes [101]. The Ku complex also binds telomeres and

462 might prevent telomere fusions [102, 103], suggesting that a low concentration of Ku on

463 Y chromosomes could also cause high rates of telomere rejoining. Third, the highly

464 repetitive nature of $Y$ chromosomes may increase the rate of DSB formation, which may

465 also contribute to a higher rate of MMEJ $[93,104]$. Fourth, we show that $Y$

466 chromosomes have high duplication and gene conversion rates, and larger deletion

467 sizes than other genomic regions (Figure 4), consistent with a preference for MMEJ to

468 repair Y-linked DSBs [93].

469 The exclusion of the Ku complex from heterochromatin could also contribute to an

470 excess of $Y$-linked duplications we observe in the $D$. simulans clade relative to $D$.

471 melanogaster (Figure 2A and 4). D. simulans clade $\mathrm{Y}$ chromosomes might harbor

472 relatively more heterochromatin than the $D$. melanogaster $Y$ due to the partial loss of

473 their euchromatic rDNA repeats [57, 61, 62], and D. simulans also expresses more

474 heterochromatin-modifying factors, such as Su(var)s and $E$ (var)s [105], compared to $D$.

475 melanogaster. To explore these hypotheses, the distribution of the Ku complex across

476 chromosomes in the testes of these species should be studied.

477 If MMEJ is preferentially used to fix DSBs on the $Y$ chromosome, we might expect that

478 the mutations in the MMEJ pathway would preferentially impact Y-bearing sperm.

479 Consistent with this prediction, a previous study showed that male $D$. melanogaster with 
480 a deficient MMEJ pathway (DNApol mutants) sire female-biased offspring [106].

481 Moreover, sperm without sex chromosomes that result from $X-Y$ non-disjunction events

482 are not as strongly affected by an MMEJ deficiency as Y-bearing sperm [106],

483 suggesting that sperm with $\mathrm{Y}$ chromosomes are more sensitive to defects in MMEJ.

484 Drosophila Y chromosomes can act as heterochromatin sinks, sequestering

485 heterochromatin marks from pericentromeric regions and suppressing position-effect

486 variegation [54, 107-109]. Therefore, retrotransposons located in heterochromatin might

487 have higher activities in males due to the presence of $Y$-linked heterochromatin [54,

488 108], although the genomic distribution of heterochromatin during spermatogenesis is

489 unknown. We find that, like D. melanogaster [22], D. simulans clade $Y$ chromosomes

490 are enriched for retrotransposons relative to the rest of the genome; however $Y$

491 chromosomes from even the closely related $D$. simulans clade species harbor distinct

492 retrotransposons (Figure S12 and Table S18), indicating that some TEs may have

493 rapidly shifted their insertion preference. This preference might benefit the TEs because

494 Y-linked TEs might express during spermatogenesis [110]. On the other hand, Y

495 chromosomes can be a significant source of small RNAs that silence repetitive

496 elements during spermatogenesis-e.g., Su(Ste) piRNAs in D. melanogaster [111,

497 112] —and thus may also contribute to TE suppression. If $Y$ chromosomes contribute to

498 piRNA or siRNA production (e.g., have piRNA clusters $[112,113]$ ), then the TE insertion

499 preference for the $\mathrm{Y}$ chromosome may sometimes be beneficial for the host, as they

500 could provide immunity against active TEs in males. In this sense, Y chromosomes may

501 even act as "TE traps" that incidentally suppress TE activity in the male germline by

502 producing small RNAs. 
503 Genes may adapt to the $\mathrm{Y}$ chromosome after residing there for millions of years [114,

504 115]. While most genes that move to the $Y$ chromosome quickly degenerate [18, 23], a

505 subset of new $\mathrm{Y}$-linked genes are retained, presumably due to important roles in male

506 fertility or sex chromosome meiotic drive. New Y-linked genes may adapt to this unique

507 genomic environment, evolving structures and regulatory mechanisms that enable

508 optimal expression on the heterochromatic and non-recombining $Y$ chromosome [116].

509 Here, we describe two new Y-linked ampliconic genes specific to the $D$. simulans

510 clade-Lhk and CK2ßtes-Y-that show evidence of strong positive evolution and

511 concerted evolution, suggesting that high copy numbers and $Y-Y$ gene conversion are

512 often important for the adaptation of new Y-linked genes.

513 Many ampliconic genes are taxonomically restricted and are not maintained at high

514 copy numbers over long periods of evolutionary time [14, 17, 20, 24-26]. Some

515 ampliconic gene families are found on both the $X$ and $Y$ chromosomes [24, 89, 117-

516 119]. While we do not know the function of most such co-amplified gene families, the

517 murine example of S/x/SIx/1 and S/y appears to be engaged in an ongoing arms race

518 between the sex chromosomes [117]. We propose that Y-linked gene amplification in

519 the $D$. simulans clade initially occurs due to an arms race and has the added benefit of

520 being preserved by gene conversion.

521 It is intriguing that the CK2ßtes-like/CK2ßtes-Y gene family is homologous to the

522 Ste/Su(Ste) system in D. melanogaster [82], which is also hypothesized to play a role in

523 sex-chromosome meiotic drive [120]. We speculate that in both the D. melanogaster

524 and $D$. simulans clade lineages these gene amplifications have been driven by conflict

525 between the sex chromosomes over transmission through meiosis, but that the conflict 
526 involves different molecular mechanisms. In the CK2ßtes-like/CK2ßtes-Y system, both

$527 \mathrm{X}$ and $\mathrm{Y}$-linked genes are protein-coding genes, which is reminiscent of S/x/S/x/1 and

528 Sly which compete for access to the nucleus where they regulate sex-linked gene

529 expression[117, 118]. In contrast, the Y-linked Su(Ste) copies in D. melanogaster

530 produce small RNAs that suppress the X-linked Stellate [84]. We propose that CK2ßtes-

531 like/CK2ßtes- $Y$ system in the $D$. simulans clade species may represent the ancestral

532 state because the parental gene $\mathrm{Ss} /$ is a protein-coding gene. We speculate that

533 systems arising from antagonisms between the sex chromosomes may shift from

534 protein-coding to RNA-based over time because, with RNAi, suppression is maintained

535 at a minimal translation cost.

536 Distinct Y-linked mutation patterns are described in many species [14-21]. Our analyses

537 provide a link between $\mathrm{Y}$-linked mutation patterns and $\mathrm{Y}$ chromosome evolution. While

538 the lack of recombination and male-limited transmission of the $Y$ chromosome reduces

539 the efficacy of selection, the high gene duplication and gene conversion rates may

540 counter these effects and help acquire and maintain new $Y$-linked genes. The unique $\mathrm{Y}$ -

541 linked mutation patterns might be the direct consequence of the heterochromatic

542 environment on sex chromosomes. Therefore, we predict that $\mathrm{W}$ chromosomes and

543 non-recombining sex-limited chromosomes (e.g., some B chromosomes), may share

544 similar mutation patterns with $\mathrm{Y}$ chromosomes. Indeed, W chromosomes of birds have

545 ampliconic genes and are rich in tandem repeats [86, 121]. However, there seem to be

546 fewer ampliconic gene families on bird W chromosomes compared to $\mathrm{Y}$ chromosomes

547 in other animals, suggesting that sexual selection and intragenomic conflict in

548 spermatogenesis are important contributors to Y-linked gene family evolution [122, 123]. 


\section{Materials and Methods}

550 Assembling Y chromosomes using Pacbio reads in D. simulans clade

551 We applied the heterochromatin-sensitive assembling pipeline from [22]. We first

552 extracted 229,464 reads with 2.2-Gbp in D. mauritiana, 269,483 reads with 2.3-Gbp in

553 D. simulans, and 257,722 reads with $2.6-$ Gbp in $D$. sechellia using assemblies from

554 [55], respectively. We then assembled these reads using Canu v1.3 and FALCON

555 v0.5.0 combined the parameter tuning method on 2 error rates, eM and eg, in bogart to

556 optimize the assemblies. We first made the Canu assemblies using the parameters

557 "genomeSize=30m stopOnReadQuality=false corMinCoverage $=0$ corOutCoverage=100

558 ovIMerSize=31" and "genomeSize=30m stopOnReadQuality=false". For FALCON

559 v0.5.0, we used the parameters "length_cutoff $=-1$; seed_coverage $=30$ or 40 ;

560 genome_size $=30000000$; length_cutoff_pr $=1000$ ”. We then picked the assemblies

561 with highest contiguity and completeness without detectable misassemblies from each

562 setting (two Canu settings and one Falcon setting).

563 After picking the three best assemblies for each species, we tentatively reconciled the

564 assemblies using Quickmerge [124]. We examined and manually curated the merged

565 assemblies. For the D. mauritiana assembly, we merged two Canu and one FALCON

566 assemblies, and for our $D$. simulans and $D$. sechellia assemblies, we merged one Canu

567 and one FALCON assemblies independently. We manually curated some conserved Y-

568 linked genes using raw reads and cDNA sequences from NCBI, including $k l-3$ of $D$.

569 mauritiana, kl-3, kl-5, and PRY of D. simulans and CCY, PRY, and Ppr-Y of D. 
570 sechellia, due to their low coverage and importance for our phylogenetic analyses. We

571 then merged our heterochromatin restricted assemblies with contigs of the major

572 chromosome arms from [55]. We polished the resulting assemblies once with Quiver

573 using PacBio reads (SMRT Analysis v2.3.0; [125] and ten times with Pilon v1.22 [126]

574 using raw Illumina reads with parameters “--mindepth 3 --minmq 10 --fix bases".

575 We identified misassemblies and found parts of $Y$-linked sequences in the contigs from

576 major arms using our female/male coverage assays in $D$. sechellia. We also assembled

577 the total reads (assuming genome size of $180 \mathrm{Mb}$ ) and heterochromatin-extracted reads

578 (assuming genome size $40 \mathrm{Mb}$ ) using wtdbg v2.4 with parameters "-x rs -t24 -X 100 -e

579 2" [127] and Flye v2.4.2 [128] with default parameters separately. We polished the

580 resulting wtdbg assemblies with raw Pacbio reads using Flye v2.4.2. We then manually

581 assembled five introns and fixed two misassemblies using sequences from wtdbg

582 whole-genome assemblies (two introns), Flye whole-genome (two introns), and

583 heterochromatin-enriched assemblies (one intron) in D. sechellia. We assembled one

584 intron using sequences from wtdbg whole-genome assemblies in $D$. simulans.

585 We also extracted potential microbial reads (except for Wolbachia) that mapped to the $D$.

586 sechellia microbial contigs, and assembled these reads into a $4.5 \mathrm{Mb}$ contig, which

587 represents the whole genome of a Providencia species, using Canu v 1.6 (r8426 588 14520f819a1e5dd221cc16553cf5b5269227b0a3) with parameters "genomeSize=5m

589 useGrid=false stopOnReadQuality=false corMinCoverage=0 corOutCoverage=100". To

590 detect other symbiont-derived sequences in our assemblies, we used Blast v2.7.1+ [129]

591 with blobtools (v1.0; [130]) to search the nt database (parameters "-task megablast - 
592 max_target_seqs 1 -max_hsps 1 -evalue 1e-25"). We estimated the Illumina coverage of

593 each contig in males for D. mauritiana, D. simulans and D. sechellia, respectively. We

594 designated and removed contigs homologous to bacteria and fungi in subsequent 595 analyses (Table S19).

Generating DNA-seq from males in the D. simulans clade

597 We extracted DNA from 30 virgin 0-day males using DNeasy Blood \& Tissue Kit and

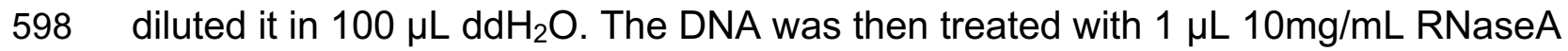

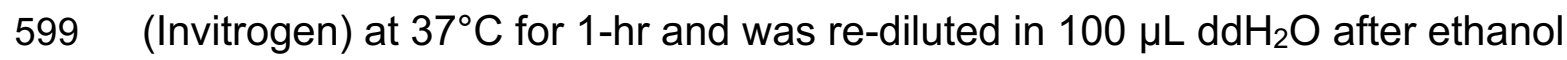

600 precipitation. The size and concentration of DNA were analyzed by gel electrophoresis,

601 Nanodrop, Qubit and Genomic DNA ScreenTape. Finally, we constructed libraries using

602 PCR-free standard Illumina kit and sequenced 125-bp paired-end reads with a 550-bp

603 insert size from the libraries using Hiseq 2500 in UR Genomics Research Center. We

604 deposited the reads in NCBI's SRA under BioProject accession number PRJNA748438.

\section{Identifying Y-linked contigs}

606 To assign contigs to the $\mathrm{Y}$ chromosome, we used Illumina reads from male and female

607 PCR-free genomic libraries (except females of $D$. mauritiana) as described in [22]. In

608 short, we mapped the male and female reads separately using BWA (v0.7.15; [131])

609 and called the coverage of uniquely mapped reads per site with samtools (v1.7; -Q 10

610 [132]). We further assigned contigs with the median of male-to-female coverage across

611 contigs equal to 0 as $\mathrm{Y}$-linked. We examined the sensitivity and specificity of our

612 methods using all 10-kb regions with known location. Based on our results for 10-kb 
613 regions with known location (Table S2) in D. mauritiana, we set up an additional

614 criterion for this species_ - "the average of female-to-male coverage $<0.1$ " — to reduce

615 the false discovery rate.

\section{Gene and repeat annotations}

617 We used the same pipeline and data to annotate genomes as a previous study [55]. We

618 collected transcripts and translated sequences from D. melanogaster (r6.14) and

619 transcript sequences from D. simulans [133] using IsoSeq3 [134]. We mapped these

620 sequences to each assembly to generate annotations using maker2 (v2.31.9; [135]. We

621 further mapped the transcriptomes using Star 2.7.3a 2-pass mapping with the maker2

622 annotation and parameters “-outFilterMultimapNmax 200 --alignSJoverhangMin 8 --

623 alignSJDBoverhangMin 1 --outFilterMismatchNmax 999 --

624 outFilterMismatchNoverReadLmax 0.04 --alignIntronMin 20 --alignIntronMax 5000000 --

625 alignMatesGapMax 5000000 --outSAMtype BAM SortedByCoordinate --

626 readFilesCommand zcat --peOverlapNbasesMin 12 --peOverlapMMp 0.1". We then

627 generated the consensus annotations using Stringtie 2.0.3 from all transcriptomes [136].

628 We further improved the mitochondria annotation using MITOS2. We assigned

629 predicted transcripts to their homologs in D. melanogaster using BLAST v2.7.1+ (-

630 evalue 1e-10; [129]).

631 We used RepeatMasker v4.0.5 [137] with our custom library to annotate the assemblies

632 using parameter "-s." Our custom library is modified from [55], by adding the consensus

633 sequence of Jockey-3 from $D$. melanogaster to replace its homologs ( $G 2$ in $D$. 
634 melanogaster and Jockey-3 in D. simulans; [138]). We extracted the sequences and

635 copies of TEs and other repeats using scripts modified from [139]. To annotate tandem

636 repeats in assemblies, we used TRFinder (v4.09; [140] with parameters "2 778010

6371002000 -ngs -h". We also used kseek to search for tandem repeats in the male

638 Illumina reads.

639 Transcriptome analyses

640 We mapped the testes transcriptome to the reference genomes of $D$. melanogaster, $D$.

641 simulans and D. mauritiana (Table S20; no available transcriptome from D. sechellia).

642 We used Stringtie 2.0.3 [136] to estimate the expression level using the annotation.

643 However, we applied a different strategy for estimating expression levels of the Y-linked

644 gene families due to the difficulties in precisely annotating multi-copies genes. We

645 constructed a transcript reference using current gene annotation but replaced all

646 transcripts from Lhk-1, Lhk-2 and CK2ßtes-Y with their species-specific reconstructed

647 ancestral copies. We then mapped the transcriptome reads to this reference using

648 Bowtie2 v 2.3.5.1 [141] with parameters “-very-sensitive -p 24 -k 200 -X 1000 --no-

649 discordant --no-mixed". We then estimated the expression level by salmon v 1.0.0 [142]

650 with parameters “-I A -p 24." We also mapped small RNA reads from D. simulans testes

651 to our custom repeat library and reconstructed ancestral Lhk-1, Lhk-2 and CK2ßtes-Y

652 sequences using Bowtie v 1.2.3 [143] with parameters “-v3 -q -a -m 50 --best -strata."

653 To assay the specific expression of different copies, we also mapped transcriptomic and

654 male genomic reads to the same reference using BWA (v0.7.15; [131]. We used ABRA 
655 v2.22 [144] to improve the alignments around the indels of these two gene families. We

656 used samtools (v1.7; [132]) to pile up reads that mapped to reconstructed ancestral

657 copies and estimated the frequency of derived SNPs in the reads.

\section{Estimating Y-linked exon copy numbers using Illumina reads}

659 We mapped the Illumina reads from the male individuals of $D$. melanogaster and the $D$.

660 simulans clade species to a genome reference with transcripts of 11 conserved $Y$-linked

661 genes and the sequences of all non-Y chromosomes (r6.14) in D. melanogaster. We

662 called the depth using samtools depth (v1.7; [132]), and estimated the copy number of

663 each exon using the mapped depth. We assumed most $Y$-linked exons are single-copy,

664 so we divided the depth of each site by the majority of depth across all Y-linked

665 transcripts to estimate the copy number. For the comparison, we simulated the 50X

666 Illumina reads from our assemblies using ART 2.5.8 with the parameter (art_illumina -ss

667 HSXt -m 500 -s 200 -p -I 150 -f 50; [145]). We then mapped the simulated reads to the

668 same reference, called the depth, and divided the depth of each site by 50.

669 Immunostaining and FISH of mitotic chromosomes

670 We conducted FISH in brain cells following the protocol from [146] and immunostaining

671 with FISH (immune-FISH) in brain cells following the protocol from [147] and [138].

672 Briefly, we dissected brains from third instar larva in 1X PBS and treated them for 1-min

673 in hypotonic solution ( $0.5 \%$ sodium citrate). Then, we fixed brain cells in $1.8 \%$

674 paraformaldehyde, $45 \%$ acetic acid for 6-min. We subsequently dehydrated in ethanol

675 for the FISH experiments but not for the immune-FISH. 
676 For immunostaining, we rehydrated the slide using PBS with $0.1 \%$ TritonX-100 after

677 removing the coverslip using liquid nitrogen. The slides were blocked with $3 \%$ BSA and

$6781 \%$ goat serum/ PBS with $0.1 \%$ TritonX-100 for 30-min and hybridized with 1:500 anti-

679 Cenp-C antibody (gift from Dr. Barbara Mellone) overnight at $4^{\circ} \mathrm{C}$. We used 1:500

680 secondary antibodies (Life Technologies Alexa-488, 546, or 647 conjugated, 1:500) in

681 blocking solution with 45-min room temperature incubation to detect the signals. We

682 fixed the slides in 4\% paraformaldehyde in 4XSSC for 6-min before doing FISH.

683 We added probes and denatured the fixed slides at $95^{\circ} \mathrm{C}$ for 5 -min and then hybridized

684 slides at $30^{\circ} \mathrm{C}$ overnight. For PCR amplified probes with DIG or biotin labels, we

685 blocked the slides for 1-hr using 3\% BSA/PBS with $0.1 \%$ Tween and incubated slides

686 with 1:200 secondary antibodies (Roche) in 3\% BSA/4X SSC with $0.1 \%$ Tween and

687 BSA at room temperature for $1 \mathrm{hr}$. We made Lhk and CK2ßtes-Y probes using PCR

688 Nick Translation kits (Roche) and ordered oligo probes from IDT. We list probe

689 information in Table S3. We mounted slides in Diamond Antifade Mountant with DAPI

690 (Invitrogen) and visualized them on a Leica DM5500 upright fluorescence microscope,

691 imaged with a Hamamatsu Orca R2 CCD camera and analyzed using Leica's LAX

692 software. We interpreted the binding patterns of $Y$ chromosomes using the density of

693 DAPI staining solely.

694 Phylogenetic analyses of Y-linked genes

695 We used BLAST v2.7.1+ [129] to extract the sequences of Y-linked duplications and

696 conserved Y-linked genes from the genome. We only used high-quality sequences 
697 polished by Pilon (--mindepth 3 --minmq 10) for our phylogenetic analyses. We aligned

698 and manually inspected sequences with reference transcripts from Flybase using

699 Geneious v8.1.6 [148]. For most Y-linked duplications, except for the genes

700 homologous to Lhk and CK2ßtes-Y, we constructed neighbor-joining trees using the

701 HKY model with 1,000 replicates using Geneious v8.1.6 [148] to infer their phylogenies.

702 We also measured the length and microhomology in 216 indels from 17 Y-linked

703 duplications using these alignments (Table S14). We also infer the potential

704 mechanisms causing the indels, including tandem duplications and polymerase slippage

705 during DNA replication. We measured the length and microhomology of polymorphic

706 indels in D. melanogaster (DGRP [95]) and D. simulans [96] populations from [55]. For

707 Lhk and CK2ßtes-Y, we constructed phylogeny using iqtree 1.6.12 [149, 150] using

708 parameters “-m MFP -nt AUTO -alrt 1000 -bb 1000 -bnni”. The node labels in Figure 5

709 correspond to SH-aLRT support (\%) / ultrafast bootstrap support (\%). The nodes with

710 SH-aLRT >= 80\% and ultrafast bootstrap support >=95\% are strongly supported.

711 Protein evolutionary rates (with CodonFreq $=0 / 1 / 2$ in PAML) of the bold branches were

712 estimated using PAML with branch models on the reconstructed ancestor sequences

713 (Fig S9 and S11).

714 Estimating recombination and selection on Y-linked ampliconic genes

715 Using the phylogenetic trees from iqtree, we infer the most probable sequences for the

716 internal nodes using MEGA $10.1 .5[151,152]$ using the maximal likelihood method and

717 G+I model with GTR model. We conducted branch and branch-site models tests in 
718 PAML 4.8 using the ancestral sequences of $Y$-linked and $X$-linked ampliconic gene

719 families with their homologs on autosomes. We plotted the tree using $\mathrm{R}$ package ape

$7205.3[153]$.

721 We used compute 0.8 .4 [154] to calculate Rmin and population recombination rates

722 based on linkage disequilibrium $[155,156]$ and gene similarity. We included sites with

723 indel polymorphisms in these analyses to increase the sample size (558-1,544 bp

724 alignments). We also reanalyzed data from Chang and Larracuente 2019 [22] to include

725 variant information from these sites. The high similarity between Y-linked ampliconic

726 gene copies may lead us to overestimate gene conversion based on gene similarity

727 [155]. We therefore also reported the lower bound on the gene conversion rate using

728 Rmin [156].

\section{GO term analysis}

730 We used PANTHER (Released 20190711; [157]) with GO Ontology database

731 (Released 2019-10-08) to perform Biological GO term analysis of new Y-linked

732 duplicated genes using Fisher's exact tests with FDR correction. We input 70 duplicated

733 genes with any known GO terms and used all genes (13767) in D. melanogaster as

734 background. 


\section{Data availability}

736 Genomic DNA sequence reads are in NCBI's SRA under BioProject PRJNA748438. All

737 scripts and pipelines are available in GitHub (forthcoming) and the Dryad digital

738 repository (doi forthcoming).

739

740

\section{Acknowledgements}

741 This work was funded by the National Institutes of Health (NIH) (R35GM119515 to

742 A.M.L. and R01GM123194 to C.D.M.), National Science Foundation (NSF MCB

743 1844693) to A.M.L. and funding from the University of Nebraska-Lincoln to C.D.M..

744 A.M.L. was supported by a Stephen Biggar and Elisabeth Asaro fellowship in Data

745 Science. C.-H.C. was supported by the Messersmith Fellowship from the $U$ of

746 Rochester, the Government Scholarship to Study Abroad from Taiwan, and the Damon

747 Runyon fellowship (DRG: 2438-21). We thank our collaborators, Drs. J.J. Emerson and

748 Mahul Chakraborty, for generating PacBio reads in the D. simulans clade, Dr. Barbara

749 Mellone for the antibodies, and Drs. Casey Bergman, Grace YC Lee, Kevin Wei and

750 Anthony Geneva and Larraucente lab members for helpful discussion. We also thank

751 the $U$ of Rochester CIRC for access to computing cluster resources and UR Genomics

752 Research Center for the library construction and sequencing. 


\section{References}

756 1. Rice WR. The Accumulation of Sexually Antagonistic Genes as a Selective Agent Promoting the Evolution of Reduced Recombination between Primitive Sex-Chromosomes. Evolution. 1987;41(4):911-4. doi: Doi 10.2307/2408899. PubMed PMID:

759 WOS:A1987J007200019.

$760 \quad 2 . \quad$ Bachtrog D. Y-chromosome evolution: emerging insights into processes of $Y$ chromosome degeneration. Nature reviews Genetics. 2013;14(2):113-24. Epub 2013/01/19. doi: 10.1038/nrg3366. PubMed PMID: 23329112; PubMed Central PMCID: PMCPMC4120474. 3. Charlesworth B. Model for evolution of $Y$ chromosomes and dosage compensation. Proceedings of the National Academy of Sciences of the United States of America. 1978;75(11):5618-22. Epub 1978/11/01. PubMed PMID: 281711; PubMed Central PMCID: PMCPMC393018.

4. Rice WR. Genetic hitchhiking and the evolution of reduced genetic activity of the $Y$ sex chromosome. Genetics. 1987;116(1):161-7. Epub 1987/05/01. PubMed PMID: 3596229; PubMed Central PMCID: PMCPMC1203114. under the background selection model. Genetics. 1995;141(4):1619-32. Epub 1995/12/01. PubMed PMID: 8601499; PubMed Central PMCID: PMCPMC1206892. Soc Lond B Biol Sci. 2000;355(1403):1563-72. Epub 2000/12/29. doi: 10.1098/rstb.2000.0717. PubMed PMID: 11127901; PubMed Central PMCID: PMCPMC1692900.

7. Bergero R, Qiu S, Charlesworth D. Gene loss from a plant sex chromosome system. Curr Biol. 2015;25(9):1234-40. Epub 2015/04/29. doi: 10.1016/j.cub.2015.03.015. PubMed PMID: 25913399.

8. Bergero R, Gardner J, Bader B, Yong L, Charlesworth D. Exaggerated heterochiasmy in a fish with sex-linked male coloration polymorphisms. Proceedings of the National Academy of Sciences of the United States of America. 2019;116(14):6924-31. Epub 2019/03/22. doi: 10.1073/pnas.1818486116. PubMed PMID: 30894479; PubMed Central PMCID: PMCPMC6452659.

9. Lenormand T, Fyon F, Sun E, Roze D. Sex Chromosome Degeneration by Regulatory Evolution. Curr Biol. 2020;30(15):3001-6 e5. Epub 2020/06/20. doi: 10.1016/j.cub.2020.05.052. PubMed PMID: 32559446.

10. Bachtrog D. Protein evolution and codon usage bias on the neo-sex chromosomes of Drosophila miranda. Genetics. 2003;165(3):1221-32. Epub 2003/12/12. PubMed PMID: 14668377 ; PubMed Central PMCID: PMCPMC1462847.

11. Singh ND, Koerich LB, Carvalho AB, Clark AG. Positive and purifying selection on the Drosophila Y chromosome. Mol Biol Evol. 2014;31(10):2612-23. Epub 2014/06/30. doi:

10.1093/molbev/msu203. PubMed PMID: 24974375; PubMed Central PMCID: PMCPMC4166921.

12. Larracuente AM, Clark AG. Surprising differences in the variability of $Y$ chromosomes in African and cosmopolitan populations of Drosophila melanogaster. Genetics. 2013;193(1):20114. Epub 2012/10/23. doi: 10.1534/genetics.112.146167. PubMed PMID: 23086221; PubMed Central PMCID: PMCPMC3527246.

13. Bachtrog D. Evidence that positive selection drives $Y$-chromosome degeneration in Drosophila miranda. Nat Genet. 2004;36(5):518-22. Epub 2004/04/27. doi: 10.1038/ng1347. PubMed PMID: 15107853.

801 14. Soh YQ, Alfoldi J, Pyntikova T, Brown LG, Graves T, Minx PJ, et al. Sequencing the 802 mouse $Y$ chromosome reveals convergent gene acquisition and amplification on both sex 
803

804

805

806

807

808

809

810

811

812

813

814

815

816

817

818

819

820

821

822

823

824

825

826

827

828

829

830

831

832

833

834

835

836

837

838

839

840

841

842

843

844

845

846

847

848

849

850

851

chromosomes. Cell. 2014;159(4):800-13. Epub 2014/11/25. doi: 10.1016/j.cell.2014.09.052. PubMed PMID: 25417157; PubMed Central PMCID: PMCPMC4260969.

15. Rozen S, Skaletsky H, Marszalek JD, Minx PJ, Cordum HS, Waterston RH, et al. Abundant gene conversion between arms of palindromes in human and ape $Y$ chromosomes. Nature. 2003;423(6942):873-6. Epub 2003/06/20. doi: 10.1038/nature01723. PubMed PMID: 12815433.

16. Hughes JF, Page DC. The Biology and Evolution of Mammalian Y Chromosomes. Annual review of genetics. 2015;49:507-27. Epub 2015/10/08. doi: 10.1146/annurev-genet112414-055311. PubMed PMID: 26442847.

17. Bachtrog D, Mahajan S, Bracewell R. Massive gene amplification on a recently formed Drosophila Y chromosome. Nat Ecol Evol. 2019;3(11):1587-97. Epub 2019/11/02. doi: 10.1038/s41559-019-1009-9. PubMed PMID: 31666742.

18. Tobler R, Nolte V, Schlotterer C. High rate of translocation-based gene birth on the Drosophila Y chromosome. Proceedings of the National Academy of Sciences of the United States of America. 2017;114(44):11721-6. Epub 2017/10/29. doi: 10.1073/pnas.1706502114. PubMed PMID: 29078298; PubMed Central PMCID: PMCPMC5676891.

19. Peichel CL, McCann SR, Ross JA, Naftaly AFS, Urton JR, Cech JN, et al. Assembly of a young vertebrate $\mathrm{Y}$ chromosome reveals convergent signatures of sex chromosome evolution. bioRxiv. 2019:2019.12.12.874701. doi: 10.1101/2019.12.12.874701.

20. Brashear WA, Raudsepp T, Murphy WJ. Evolutionary conservation of Y Chromosome ampliconic gene families despite extensive structural variation. Genome research. 2018;28(12):1841-51. Epub 2018/11/02. doi: 10.1101/gr.237586.118. PubMed PMID: 30381290; PubMed Central PMCID: PMCPMC6280758.

21. Hall AB, Papathanos PA, Sharma A, Cheng C, Akbari OS, Assour L, et al. Radical remodeling of the $Y$ chromosome in a recent radiation of malaria mosquitoes. Proceedings of the National Academy of Sciences of the United States of America. 2016;113(15):E2114-23. Epub 2016/04/02. doi: 10.1073/pnas.1525164113. PubMed PMID: 27035980; PubMed Central PMCID: PMCPMC4839409.

22. Chang $\mathrm{CH}$, Larracuente AM. Heterochromatin-Enriched Assemblies Reveal the Sequence and Organization of the Drosophila melanogaster Y Chromosome. Genetics. 2019;211(1):333-48. Epub 2018/11/14. doi: 10.1534/genetics.118.301765. PubMed PMID: 30420487; PubMed Central PMCID: PMCPMC6325706.

23. Carvalho AB, Vicoso B, Russo CA, Swenor B, Clark AG. Birth of a new gene on the $Y$ chromosome of Drosophila melanogaster. Proceedings of the National Academy of Sciences of the United States of America. 2015;112(40):12450-5. Epub 2015/09/20. doi: 10.1073/pnas.1516543112. PubMed PMID: 26385968; PubMed Central PMCID: PMCPMC4603513.

24. Ellison C, Bachtrog D. Recurrent gene co-amplification on Drosophila $X$ and $Y$ chromosomes. PLoS Genet. 2019;15(7):e1008251. Epub 2019/07/23. doi: 10.1371/journal.pgen.1008251. PubMed PMID: 31329593; PubMed Central PMCID: PMCPMC6690552.

25. Hughes JF, Skaletsky H, Pyntikova T, Graves TA, van Daalen SK, Minx PJ, et al. Chimpanzee and human $\mathrm{Y}$ chromosomes are remarkably divergent in structure and gene content. Nature. 2010;463(7280):536-9. Epub 2010/01/15. doi: 10.1038/nature08700. PubMed PMID: 20072128; PubMed Central PMCID: PMCPMC3653425.

26. Mueller JL, Mahadevaiah SK, Park PJ, Warburton PE, Page DC, Turner JM. The mouse $\mathrm{X}$ chromosome is enriched for multicopy testis genes showing postmeiotic expression. Nat Genet. 2008;40(6):794-9. Epub 2008/05/06. doi: 10.1038/ng.126. PubMed PMID: 18454149; PubMed Central PMCID: PMCPMC2740655. 
852

853

854

855

856

857

858

859

860

861

862

863

864

865

866

867

868

869

870

871

872

873

874

875

876

877

878

879

880

881

882

883

884

885

886

887

888

889

890

891

892

893

894

895

896

897

898

899

900

901

27. Connallon T, Clark AG. Gene duplication, gene conversion and the evolution of the $Y$ chromosome. Genetics. 2010;186(1):277-86. doi: 10.1534/genetics.110.116756. PubMed PMID: 20551442; PubMed Central PMCID: PMCPMC2940292.

28. Carlson M, Brutlag D. Cloning and characterization of a complex satellite DNA from Drosophila melanogaster. Cell. 1977;11(2):371-81. Epub 1977/06/01. PubMed PMID: 408008. 29. Lohe AR, Brutlag DL. Identical satellite DNA sequences in sibling species of Drosophila. J Mol Biol. 1987;194(2):161-70. Epub 1987/03/20. PubMed PMID: 3112413.

30. Lohe AR, Brutlag DL. Adjacent satellite DNA segments in Drosophila structure of junctions. J Mol Biol. 1987;194(2):171-9. Epub 1987/03/20. PubMed PMID: 3112414.

31. Mahajan S, Wei KH, Nalley MJ, Gibilisco L, Bachtrog D. De novo assembly of a young Drosophila $Y$ chromosome using single-molecule sequencing and chromatin conformation capture. PLoS Biol. 2018;16(7):e2006348. Epub 2018/07/31. doi: 10.1371/journal.pbio.2006348. PubMed PMID: 30059545; PubMed Central PMCID: PMCPMC6117089.

32. Araripe LO, Tao Y, Lemos B. Interspecific $Y$ chromosome variation is sufficient to rescue hybrid male sterility and is influenced by the grandparental origin of the chromosomes. Heredity (Edinb). 2016;116(6):516-22. Epub 2016/03/17. doi: 10.1038/hdy.2016.11. PubMed PMID: 26980343; PubMed Central PMCID: PMCPMC4868264.

33. Bayes JJ, Malik HS. Altered heterochromatin binding by a hybrid sterility protein in Drosophila sibling species. Science. 2009;326(5959):1538-41. Epub 2009/11/26. doi: 10.1126/science.1181756. PubMed PMID: 19933102; PubMed Central PMCID: PMCPMC2987944.

34. Johnson NA, Perez DE, Cabot EL, Hollocher H, Wu Cl. A test of reciprocal X-Y interactions as a cause of hybrid sterility in Drosophila. Nature. 1992;358(6389):751-3. Epub 1992/08/27. doi: 10.1038/358751a0. PubMed PMID: 1508270.

35. Coyne JA. The genetic basis of Haldane's rule. Nature. 1985;314(6013):736-8. Epub 1985/04/01. doi: 10.1038/314736a0. PubMed PMID: 3921852.

36. Bozzetti MP, Massari S, Finelli P, Meggio F, Pinna LA, Boldyreff B, et al. The Ste locus, a component of the parasitic cry-Ste system of Drosophila melanogaster, encodes a protein that forms crystals in primary spermatocytes and mimics properties of the beta subunit of casein kinase 2. Proceedings of the National Academy of Sciences of the United States of America. 1995;92(13):6067-71. Epub 1995/06/20. PubMed PMID: 7597082; PubMed Central PMCID: PMCPMC41643.

37. Courret $\mathrm{C}$, Chang $\mathrm{CH}$, Wei KH, Montchamp-Moreau C, Larracuente AM. Meiotic drive mechanisms: lessons from Drosophila. Proc Biol Sci. 2019;286(1913):20191430. Epub 2019/10/24. doi: 10.1098/rspb.2019.1430. PubMed PMID: 31640520; PubMed Central PMCID: PMCPMC6834043.

38. Tao Y, Araripe L, Kingan SB, Ke Y, Xiao H, Hartl DL. A sex-ratio meiotic drive system in Drosophila simulans. II: an X-linked distorter. PLoS Biol. 2007;5(11):e293. Epub 2007/11/09. doi: 10.1371/journal.pbio.0050293. PubMed PMID: 17988173; PubMed Central PMCID: PMCPMC2062476.

39. Tao Y, Hartl DL, Laurie CC. Sex-ratio segregation distortion associated with reproductive isolation in Drosophila. Proceedings of the National Academy of Sciences of the United States of America. 2001;98(23):13183-8. Epub 2001/11/01. doi: 10.1073/pnas.231478798. PubMed PMID: 11687638; PubMed Central PMCID: PMCPMC60845.

40. Helleu Q, Courret C, Ogereau D, Burnham KL, Chaminade N, Chakir M, et al. Sex-Ratio Meiotic Drive Shapes the Evolution of the Y Chromosome in Drosophila simulans. Mol Biol Evol. 2019;36(12):2668-81. Epub 2019/07/11. doi: 10.1093/molbev/msz160. PubMed PMID: 31290972.

41. Branco AT, Tao Y, Hartl DL, Lemos B. Natural variation of the $Y$ chromosome suppresses sex ratio distortion and modulates testis-specific gene expression in Drosophila 
902

903

904

905

906

907

908

909

910

911

912

913

914

915

916

917

918

919

920

921

922

923

924

925

926

927

928

929

930

931

932

933

934

935

936

937

938

939

940

941

942

943

944

945

946

947

948

949

950

951

simulans. Heredity (Edinb). 2013;111(1):8-15. Epub 2013/04/18. doi: 10.1038/hdy.2013.5. PubMed PMID: 23591516; PubMed Central PMCID: PMCPMC3692315.

42. Montchamp-Moreau C, Ginhoux V, Atlan A. The Y chromosomes of Drosophila simulans are highly polymorphic for their ability to suppress sex-ratio drive. Evolution. 2001;55(4):728-37. Epub 2001/06/08. PubMed PMID: 11392391.

43. Meiklejohn CD, Landeen EL, Gordon KE, Rzatkiewicz T, Kingan SB, Geneva AJ, et al. Gene flow mediates the role of sex chromosome meiotic drive during complex speciation. Elife. 2018;7. Epub 2018/12/14. doi: 10.7554/eLife.35468. PubMed PMID: 30543325; PubMed Central PMCID: PMCPMC6292695.

44. Reijo R, Lee TY, Salo P, Alagappan R, Brown LG, Rosenberg M, et al. Diverse spermatogenic defects in humans caused by $Y$ chromosome deletions encompassing a novel RNA-binding protein gene. Nat Genet. 1995;10(4):383-93. Epub 1995/08/01. doi: 10.1038/ng0895-383. PubMed PMID: 7670487.

45. Vogt PH, Edelmann A, Kirsch S, Henegariu O, Hirschmann P, Kiesewetter F, et al. Human Y chromosome azoospermia factors (AZF) mapped to different subregions in Yq11. Hum Mol Genet. 1996;5(7):933-43. Epub 1996/07/01. PubMed PMID: 8817327.

46. Sun C, Skaletsky H, Rozen S, Gromoll J, Nieschlag E, Oates R, et al. Deletion of azoospermia factor a (AZFa) region of human $\mathrm{Y}$ chromosome caused by recombination between HERV15 proviruses. Hum Mol Genet. 2000;9(15):2291-6. Epub 2000/09/26. PubMed PMID: 11001932.

47. Repping S, Skaletsky H, Brown L, van Daalen SK, Korver CM, Pyntikova T, et al. Polymorphism for a 1.6-Mb deletion of the human $\mathrm{Y}$ chromosome persists through balance between recurrent mutation and haploid selection. Nat Genet. 2003;35(3):247-51. Epub 2003/10/07. doi: 10.1038/ng1250. PubMed PMID: 14528305.

48. Morgan AP, Pardo-Manuel de Villena F. Sequence and Structural Diversity of Mouse $Y$ Chromosomes. Mol Biol Evol. 2017;34(12):3186-204. Epub 2017/10/14. doi:

10.1093/molbev/msx250. PubMed PMID: 29029271.

49. Lemos B, Branco AT, Hartl DL. Epigenetic effects of polymorphic $Y$ chromosomes modulate chromatin components, immune response, and sexual conflict. Proceedings of the National Academy of Sciences of the United States of America. 2010;107(36):15826-31. Epub 2010/08/28. doi: 10.1073/pnas.1010383107. PubMed PMID: 20798037; PubMed Central PMCID: PMCPMC2936610.

50. Wang M, Branco AT, Lemos B. The Y Chromosome Modulates Splicing and Sex-Biased Intron Retention Rates in Drosophila. Genetics. 2017. Epub 2017/12/22. doi:

10.1534/genetics.117.300637. PubMed PMID: 29263027.

51. Sackton TB, Montenegro H, Hartl DL, Lemos B. Interspecific Y chromosome introgressions disrupt testis-specific gene expression and male reproductive phenotypes in Drosophila. Proceedings of the National Academy of Sciences of the United States of America. 2011;108(41):17046-51. Epub 2011/10/05. doi: 10.1073/pnas.1114690108. PubMed PMID: 21969588; PubMed Central PMCID: PMCPMC3193250.

52. Zhou J, Sackton TB, Martinsen L, Lemos B, Eickbush TH, Hartl DL. Y chromosome mediates ribosomal DNA silencing and modulates the chromatin state in Drosophila. Proceedings of the National Academy of Sciences of the United States of America. 2012;109(25):9941-6. Epub 2012/06/06. doi: 10.1073/pnas.1207367109. PubMed PMID: 22665801; PubMed Central PMCID: PMCPMC3382510.

53. Case LK, Wall EH, Osmanski EE, Dragon JA, Saligrama N, Zachary JF, et al. Copy number variation in $\mathrm{Y}$ chromosome multicopy genes is linked to a paternal parent-of-origin effect on CNS autoimmune disease in female offspring. Genome Biol. 2015;16:28. Epub 2015/04/19. doi: 10.1186/s13059-015-0591-7. PubMed PMID: 25886764; PubMed Central PMCID: PMCPMC4396973. 
952

953

954

955

956

957

958

959

960

961

962

963

964

965

966

967

968

969

970

971

972

973

974

975

976

977

978

979

980

981

982

983

984

985

986

987

988

989

990

991

992

993

994

995

996

997

998

999

1000

1001

54. Brown E, Bachtrog D. The Drosophila Y chromosome affects heterochromatin integrity genome-wide. bioRxiv. 2017. doi: 10.1101/156000.

55. Chakraborty M, Chang CH, Khost DE, Vedanayagam J, Adrion JR, Liao Y, et al. Evolution of genome structure in the Drosophila simulans species complex. Genome research. 2021;31(3):380-96. Epub 2021/02/11. doi: 10.1101/gr.263442.120. PubMed PMID: 33563718; PubMed Central PMCID: PMCPMC7919458.

56. Lemeunier F, Ashburner M. Relationships within the melanogaster species subgroup of the genus Drosophila (Sophophora). Chromosoma. 1984;89(5):343-51. doi:

$10.1007 / \mathrm{bf00331251.}$

57. Roy V, Monti-Dedieu L, Chaminade N, Siljak-Yakovlev S, Aulard S, Lemeunier F, et al. Evolution of the chromosomal location of rDNA genes in two Drosophila species subgroups: ananassae and melanogaster. Heredity (Edinb). 2005;94(4):388-95. Epub 2005/02/24. doi: 10.1038/sj.hdy.6800612. PubMed PMID: 15726113.

58. Berloco M, Fanti L, Sheen F, Levis RW, Pimpinelli S. Heterochromatic distribution of HeT-A- and TART-like sequences in several Drosophila species. Cytogenetic and genome research. 2005;110(1-4):124-33. Epub 2005/08/12. doi: 10.1159/000084944. PubMed PMID: 16093664.

59. Erhardt S, Mellone BG, Betts CM, Zhang W, Karpen GH, Straight AF. Genome-wide analysis reveals a cell cycle-dependent mechanism controlling centromere propagation. J Cell Biol. 2008;183(5):805-18. Epub 2008/12/03. doi: 10.1083/jcb.200806038. PubMed PMID: 19047461; PubMed Central PMCID: PMCPMC2592830.

60. Paredes S, Branco AT, Hartl DL, Maggert KA, Lemos B. Ribosomal DNA deletions modulate genome-wide gene expression: "rDNA-sensitive" genes and natural variation. PLoS Genet. 2011;7(4):e1001376. Epub 2011/05/03. doi: 10.1371/journal.pgen.1001376. PubMed PMID: 21533076; PubMed Central PMCID: PMCPMC3080856.

61. Lohe AR, Roberts PA. Evolution of DNA in heterochromatin: the Drosophila melanogaster sibling species subgroup as a resource. Genetica. 2000;109(1-2):125-30. Epub 2001/04/11. PubMed PMID: 11293787.

62. Lohe AR, Roberts PA. An unusual Y chromosome of Drosophila simulans carrying amplified rDNA spacer without rRNA genes. Genetics. 1990;125(2):399-406. Epub 1990/06/01. PubMed PMID: 2379820; PubMed Central PMCID: PMCPMC1204028.

63. McKee BD, Karpen GH. Drosophila ribosomal RNA genes function as an X-Y pairing site during male meiosis. Cell. 1990;61(1):61-72. Epub 1990/04/06. PubMed PMID: 2156630.

64. Kopp A, Frank A, Fu J. Historical biogeography of Drosophila simulans based on Ychromosomal sequences. Mol Phylogenet Evol. 2006;38(2):355-62. Epub 2005/07/30. doi: 10.1016/j.ympev.2005.06.006. PubMed PMID: 16051503.

65. Chakraborty M, Chang C-H, Khost D, Vedanayagam J, Adrion JR, Liao Y, et al. Evolution of genome structure in the Drosophila simulans species complex. bioRxiv. 2020. 66. Bonaccorsi S, Pisano C, Puoti F, Gatti M. Y chromosome loops in Drosophila melanogaster. Genetics. 1988;120(4):1015-34. Epub 1988/12/01. PubMed PMID: 2465201; PubMed Central PMCID: PMCPMC1203565.

67. Bonaccorsi S, Gatti M, Pisano C, Lohe A. Transcription of a satellite DNA on two Y chromosome loops of Drosophila melanogaster. Chromosoma. 1990;99(4):260-6. Epub 1990/08/01. PubMed PMID: 2119983.

68. Meyer GnF. Die Funktionsstrukturen des Y-Chromosoms in den Spermatocytenkernen von Drosophila hydei, D. neohydei, D. repleta und einigen anderen Drosophila-Arten. Chromosoma. 1963;14(3):207-55. doi: 10.1007/bf00326814.

69. Piergentili R. Evolutionary conservation of lampbrush-like loops in drosophilids. BMC Cell Biol. 2007;8:35. Epub 2007/08/19. doi: 10.1186/1471-2121-8-35. PubMed PMID: 17697358; PubMed Central PMCID: PMCPMC1978495. 
1002

1003

1004

1005

1006

1007

1008

1009

1010

1011

1012

1013

1014

1015

1016

1017

1018

1019

1020

1021

1022

1023

1024

1025

1026

1027

1028

1029

1030

1031

1032

1033

1034

1035

1036

1037

1038

1039

1040

1041

1042

1043

1044

1045

1046

1047

1048

1049

1050

1051

1052
70. Fingerhut JM, Moran JV, Yamashita YM. Satellite DNA-containing gigantic introns in a unique gene expression program during Drosophila spermatogenesis. PLoS Genet.

2019;15(5):e1008028. Epub 2019/05/10. doi: 10.1371/journal.pgen.1008028. PubMed PMID: 31071079; PubMed Central PMCID: PMCPMC6508621.

71. Redhouse JL, Mozziconacci J, White RA. Co-transcriptional architecture in a $Y$ loop in Drosophila melanogaster. Chromosoma. 2011;120(4):399-407. Epub 2011/05/11. doi: 10.1007/s00412-011-0321-1. PubMed PMID: 21556802.

72. Pisano C, Bonaccorsi S, Gatti M. The kl-3 loop of the Y chromosome of Drosophila melanogaster binds a tektin-like protein. Genetics. 1993;133(3):569-79. Epub 1993/03/01. PubMed PMID: 8454204; PubMed Central PMCID: PMCPMC1205344.

73. Piergentili R, Bonaccorsi S, Raffa GD, Pisano C, Hackstein JH, Mencarelli C. Autosomal control of the Y-chromosome kl-3 loop of Drosophila melanogaster. Chromosoma. 2004;113(4):188-96. Epub 2004/09/01. doi: 10.1007/s00412-004-0308-2. PubMed PMID: 15338233.

74. Piergentili R, Mencarelli C. Drosophila melanogaster kl-3 and kl-5 Y-loops harbor triplestranded nucleic acids. J Cell Sci. 2008;121(Pt 10):1605-12. Epub 2008/04/24. doi: 10.1242/jcs.025320. PubMed PMID: 18430782.

75. Chang $\mathrm{CH}$, Larracuente AM. Genomic changes following the reversal of a $Y$ chromosome to an autosome in Drosophila pseudoobscura. Evolution. 2017;71(5):1285-96. Epub 2017/03/23. doi: 10.1111/evo.13229. PubMed PMID: 28322435; PubMed Central PMCID: PMCPMC5485016.

76. Jagannathan M, Warsinger-Pepe N, Watase GJ, Yamashita YM. Comparative Analysis of Satellite DNA in the Drosophila melanogaster Species Complex. G3 (Bethesda).

2017;7(2):693-704. Epub 2016/12/23. doi: 10.1534/g3.116.035352. PubMed PMID: 28007840; PubMed Central PMCID: PMCPMC5295612.

77. Mahajan S, Bachtrog D. Convergent evolution of $\mathrm{Y}$ chromosome gene content in flies. Nat Commun. 2017;8(1):785. Epub 2017/10/06. doi: 10.1038/s41467-017-00653-x. PubMed PMID: 28978907; PubMed Central PMCID: PMCPMC5627270.

78. Greil F, Ahmad K. Nucleolar dominance of the $\mathrm{Y}$ chromosome in Drosophila melanogaster. Genetics. 2012;191(4):1119-28. Epub 2012/06/01. doi:

10.1534/genetics.112.141242. PubMed PMID: 22649076; PubMed Central PMCID: PMCPMC3415996.

79. Mahadevaraju S, Fear JM, Akeju M, Galletta BJ, Pinheiro M, Avelino CC, et al. Dynamic sex chromosome expression in Drosophila male germ cells. Nat Commun. 2021;12(1):892. Epub 2021/02/11. doi: 10.1038/s41467-021-20897-y. PubMed PMID: 33563972; PubMed Central PMCID: PMCPMC7873209.

80. Hess O, Meyer GF. Genetic activities of the $Y$ chromosome in Drosophila during spermatogenesis. Adv Genet. 1968;14:171-223. Epub 1968/01/01. doi: 10.1016/s00652660(08)60427-7. PubMed PMID: 4884781.

81. Loh BJ, Cullen CF, Vogt N, Ohkura H. The conserved kinase SRPK regulates karyosome formation and spindle microtubule assembly in Drosophila oocytes. J Cell Sci. 2012;125(Pt 19):4457-62. Epub 2012/08/03. doi: 10.1242/jcs.107979. PubMed PMID: 22854045; PubMed Central PMCID: PMCPMC3500864.

82. Kogan GL, Usakin LA, Ryazansky SS, Gvozdev VA. Expansion and evolution of the Xlinked testis specific multigene families in the melanogaster species subgroup. PLoS One. 2012;7(5):e37738. Epub 2012/06/01. doi: 10.1371/journal.pone.0037738. PubMed PMID: 22649555; PubMed Central PMCID: PMCPMC3359341.

83. Danilevskaya ON, Kurenova EV, Pavlova MN, Bebehov DV, Link AJ, Koga A, et al. He-T family DNA sequences in the $Y$ chromosome of Drosophila melanogaster share homology with the X-linked stellate genes. Chromosoma. 1991;100(2):118-24. Epub 1991/02/01. PubMed PMID: 1672635. 
84. Aravin AA, Klenov MS, Vagin VV, Bantignies F, Cavalli G, Gvozdev VA. Dissection of a natural RNA silencing process in the Drosophila melanogaster germ line. Mol Cell Biol. 2004;24(15):6742-50. Epub 2004/07/16. doi: 10.1128/MCB.24.15.6742-6750.2004. PubMed PMID: 15254241; PubMed Central PMCID: PMCPMC444866.

85. Ohta T. Some models of gene conversion for treating the evolution of multigene families. Genetics. 1984;106(3):517-28. PubMed PMID: 6706111; PubMed Central PMCID: PMCPMC1224254.

86. Backstrom N, Ceplitis H, Berlin S, Ellegren H. Gene conversion drives the evolution of HINTW, an ampliconic gene on the female-specific avian W chromosome. Mol Biol Evol. 2005;22(10):1992-9. doi: 10.1093/molbev/msi198. PubMed PMID: 15972846.

87. Yang Z. PAML: a program package for phylogenetic analysis by maximum likelihood. Comput Appl Biosci. 1997;13(5):555-6. Epub 1997/11/21. PubMed PMID: 9367129.

88. Dover G. Molecular drive: a cohesive mode of species evolution. Nature. 1982;299(5879):111-7. Epub 1982/09/09. doi: 10.1038/299111a0. PubMed PMID: 7110332. 89. Malone CD, Lehmann R, Teixeira FK. The cellular basis of hybrid dysgenesis and Stellate regulation in Drosophila. Curr Opin Genet Dev. 2015;34:88-94. Epub 2015/10/10. doi: 10.1016/j.gde.2015.09.003. PubMed PMID: 26451497; PubMed Central PMCID: PMCPMC4674331.

90. Palumbo G, Bonaccorsi S, Robbins LG, Pimpinelli S. Genetic analysis of Stellate elements of Drosophila melanogaster. Genetics. 1994;138(4):1181-97. Epub 1994/12/01. PubMed PMID: 7896100; PubMed Central PMCID: PMCPMC1206257.

91. Meyer GF, Hess O, Beermann W. Phasenspezifische Funktionsstrukturen in Spermatocytenkernen von Drosophila melanogaster und Ihre Abhängigkeit vom Y-Chromosom. Chromosoma. 1961;12(1):676-716. doi: 10.1007/BF00328946.

92. Chan SH, Yu AM, McVey M. Dual roles for DNA polymerase theta in alternative endjoining repair of double-strand breaks in Drosophila. PLoS Genet. 2010;6(7):e1001005. Epub 2010/07/10. doi: 10.1371/journal.pgen.1001005. PubMed PMID: 20617203; PubMed Central PMCID: PMCPMC2895639.

93. McVey M, Lee SE. MMEJ repair of double-strand breaks (director's cut): deleted sequences and alternative endings. Trends Genet. 2008;24(11):529-38. Epub 2008/09/24. doi: 10.1016/j.tig.2008.08.007. PubMed PMID: 18809224; PubMed Central PMCID: PMCPMC5303623.

94. Chang HHY, Pannunzio NR, Adachi N, Lieber MR. Non-homologous DNA end joining and alternative pathways to double-strand break repair. Nat Rev Mol Cell Biol. 2017;18(8):495506. Epub 2017/05/18. doi: 10.1038/nrm.2017.48. PubMed PMID: 28512351.

95. Huang W, Massouras A, Inoue Y, Peiffer J, Ramia M, Tarone AM, et al. Natural variation in genome architecture among 205 Drosophila melanogaster Genetic Reference Panel lines. Genome research. 2014;24(7):1193-208. Epub 2014/04/10. doi: 10.1101/gr.171546.113. PubMed PMID: 24714809; PubMed Central PMCID: PMCPMC4079974.

96. Signor SA, New FN, Nuzhdin S. A Large Panel of Drosophila simulans Reveals an Abundance of Common Variants. Genome biology and evolution. 2018;10(1):189-206. doi: 10.1093/gbe/evx262. PubMed PMID: 29228179; PubMed Central PMCID: PMCPMC5767965. 97. Wei KHC, Lower SE, Caldas IV, Sless TJ, Barbash DA, Clark AG. Variable rates of simple satellite gains across the Drosophila phylogeny. Molecular Biology and Evolution. 2018:msy005-msy. doi: 10.1093/molbev/msy005.

98. Cechova M, Harris RS, Tomaszkiewicz M, Arbeithuber B, Chiaromonte F, Makova KD. High satellite repeat turnover in great apes studied with short- and long-read technologies. Mol Biol Evol. 2019. Epub 2019/07/06. doi: 10.1093/molbev/msz156. PubMed PMID: 31273383; PubMed Central PMCID: PMCPMC6805231.

99. Abad JP, de Pablos B, Agudo M, Molina I, Giovinazzo G, Martin-Gallardo A, et al. Genomic and cytological analysis of the $\mathrm{Y}$ chromosome of Drosophila melanogaster: telomere- 
1104

1105

1106

1107

1108

1109

1110

1111

1112

1113

1114

1115

1116

1117

1118

1119

1120

1121

1122

1123

1124

1125

1126

1127

1128

1129

1130

1131

1132

1133

1134

1135

1136

1137

1138

1139

1140

1141

1142

1143

1144

1145

1146

1147

1148

1149

1150

1151

1152

1153

1154

derived sequences at internal regions. Chromosoma. 2004;113(6):295-304. Epub 2004/12/24. doi: 10.1007/s00412-004-0318-0. PubMed PMID: 15616866.

100. Agudo M, Losada A, Abad JP, Pimpinelli S, Ripoll P, Villasante A. Centromeres from telomeres? The centromeric region of the $Y$ chromosome of Drosophila melanogaster contains a tandem array of telomeric HeT-A- and TART-related sequences. Nucleic Acids Res. 1999;27(16):3318-24. Epub 1999/08/24. PubMed PMID: 10454639; PubMed Central PMCID: PMCPMC148565.

101. Chiolo I, Minoda A, Colmenares SU, Polyzos A, Costes SV, Karpen GH. Double-strand breaks in heterochromatin move outside of a dynamic HP1a domain to complete recombinational repair. Cell. 2011;144(5):732-44. Epub 2011/03/01. doi: 10.1016/j.cell.2011.02.012. PubMed PMID: 21353298; PubMed Central PMCID: PMCPMC3417143.

102. Melnikova L, Biessmann $\mathrm{H}$, Georgiev $\mathrm{P}$. The Ku protein complex is involved in length regulation of Drosophila telomeres. Genetics. 2005;170(1):221-35. Epub 2005/03/23. doi: 10.1534/genetics.104.034538. PubMed PMID: 15781709; PubMed Central PMCID: PMCPMC1449706.

103. Samper E, Goytisolo FA, Slijepcevic P, van Buul PP, Blasco MA. Mammalian Ku86 protein prevents telomeric fusions independently of the length of TTAGGG repeats and the Gstrand overhang. EMBO reports. 2000;1(3):244-52. Epub 2001/03/21. doi: 10.1093/emboreports/kvd051. PubMed PMID: 11256607; PubMed Central PMCID: PMCPMC1083725.

104. Katsura Y, Sasaki S, Sato M, Yamaoka K, Suzukawa K, Nagasawa T, et al. Involvement of Ku80 in microhomology-mediated end joining for DNA double-strand breaks in vivo. DNA Repair (Amst). 2007;6(5):639-48. Epub 2007/01/24. doi: 10.1016/j.dnarep.2006.12.002. PubMed PMID: 17236818.

105. Lee YCG, Karpen GH. Pervasive epigenetic effects of Drosophila euchromatic transposable elements impact their evolution. Elife. 2017;6. Epub 2017/07/12. doi: 10.7554/eLife.25762. PubMed PMID: 28695823; PubMed Central PMCID: PMCPMC5505702. 106. McKee BD, Hong CS, Das S. On the roles of heterochromatin and euchromatin in meiosis in drosophila: mapping chromosomal pairing sites and testing candidate mutations for effects on X-Y nondisjunction and meiotic drive in male meiosis. Genetica. 2000;109(1-2):77-93. Epub 2001/04/11. PubMed PMID: 11293799.

107. Dimitri P, Pisano C. Position effect variegation in Drosophila melanogaster: relationship between suppression effect and the amount of Y chromosome. Genetics. 1989;122(4):793-800. Epub 1989/08/01. PubMed PMID: 2503420; PubMed Central PMCID: PMCPMC1203755.

108. Henikoff S. Dosage-dependent modification of position-effect variegation in Drosophila. BioEssays : news and reviews in molecular, cellular and developmental biology. 1996;18(5):401-9. Epub 1996/05/01. doi: 10.1002/bies.950180510. PubMed PMID: 8639163. 109. Gatti M, Pimpinelli S. Functional elements in Drosophila melanogaster heterochromatin. Annual review of genetics. 1992;26:239-75. Epub 1992/01/01. doi: 10.1146/annurev.ge.26.120192.001323. PubMed PMID: 1482113.

110. Lawlor MA, Cao W, Ellison CE. A burst of transposon expression accompanies the activation of $Y$ chromosome fertility genes during Drosophila spermatogenesis. bioRxiv. 2021:2021.05.10.443472. doi: 10.1101/2021.05.10.443472.

111. Quenerch'du E, Anand A, Kai T. The piRNA pathway is developmentally regulated during spermatogenesis in Drosophila. RNA. 2016;22(7):1044-54. Epub 2016/05/22. doi: 10.1261/rna.055996.116. PubMed PMID: 27208314; PubMed Central PMCID: PMCPMC4911912.

112. Aravin AA, Naumova NM, Tulin AV, Vagin VV, Rozovsky YM, Gvozdev VA. Doublestranded RNA-mediated silencing of genomic tandem repeats and transposable elements in the D. melanogaster germline. Curr Biol. 2001;11(13):1017-27. Epub 2001/07/27. PubMed PMID: 11470406. 
113. Chen P, Kotov AA, Godneeva BK, Bazylev SS, Olenina LV, Aravin AA. piRNA-mediated gene regulation and adaptation to sex-specific transposon expression in D. melanogaster male germline. Genes Dev. 2021;35(11-12):914-35. Epub 2021/05/15. doi: 10.1101/gad.345041.120. PubMed PMID: 33985970; PubMed Central PMCID: PMCPMC8168559.

114. Wakimoto BT, Hearn MG. The effects of chromosome rearrangements on the expression of heterochromatic genes in chromosome $2 \mathrm{~L}$ of Drosophila melanogaster. Genetics. 1990;125(1):141-54. Epub 1990/05/01. PubMed PMID: 2111264; PubMed Central PMCID: PMCPMC1203996.

115. Hearn MG, Hedrick A, Grigliatti TA, Wakimoto BT. The effect of modifiers of positioneffect variegation on the variegation of heterochromatic genes of Drosophila melanogaster. Genetics. 1991;128(4):785-97. Epub 1991/08/01. PubMed PMID: 1916244; PubMed Central PMCID: PMCPMC1204552.

116. Dupim EG, Goldstein G, Vanderlinde T, Vaz SC, Krsticevic F, Bastos A, et al. An investigation of $Y$ chromosome incorporations in 400 species of Drosophila and related genera. PLoS Genet. 2018;14(11):e1007770. Epub 2018/11/06. doi: 10.1371/journal.pgen.1007770. PubMed PMID: 30388103; PubMed Central PMCID: PMCPMC6235401. 117. Cocquet J, Ellis PJ, Mahadevaiah SK, Affara NA, Vaiman D, Burgoyne PS. A genetic basis for a postmeiotic $X$ versus $Y$ chromosome intragenomic conflict in the mouse. PLoS Genet. 2012;8(9):e1002900. Epub 2012/10/03. doi: 10.1371/journal.pgen.1002900. PubMed PMID: 23028340; PubMed Central PMCID: PMCPMC3441658.

118. Kruger AN, Brogley MA, Huizinga JL, Kidd JM, de Rooij DG, Hu YC, et al. A Neofunctionalized X-Linked Ampliconic Gene Family Is Essential for Male Fertility and Equal Sex Ratio in Mice. Curr Biol. 2019;29(21):3699-706 e5. Epub 2019/10/22. doi: 10.1016/j.cub.2019.08.057. PubMed PMID: 31630956.

119. Lahn BT, Page DC. A human sex-chromosomal gene family expressed in male germ cells and encoding variably charged proteins. Hum Mol Genet. 2000;9(2):311-9. Epub 1999/12/23. doi: 10.1093/hmg/9.2.311. PubMed PMID: 10607842.

120. Hurst LD. Is Stellate a relict meiotic driver? Genetics. 1992;130(1):229-30. Epub 1992/01/01. PubMed PMID: 1732164; PubMed Central PMCID: PMCPMC1204797.

121. Komissarov AS, Galkina SA, Koshel El, Kulak MM, Dyomin AG, O'Brien SJ, et al. New high copy tandem repeat in the content of the chicken W chromosome. Chromosoma. 2018;127(1):73-83. Epub 2017/09/28. doi: 10.1007/s00412-017-0646-5. PubMed PMID: 28951974.

122. Bachtrog $D$. The $Y$ Chromosome as a Battleground for Intragenomic Conflict. Trends Genet. 2020;36(7):510-22. Epub 2020/05/26. doi: 10.1016/j.tig.2020.04.008. PubMed PMID: 32448494.

123. Rogers MJ. Y chromosome copy number variation and its effects on fertility and other health factors: a review. Transl Androl Urol. 2021;10(3):1373-82. Epub 2021/04/15. doi: 10.21037/tau.2020.04.06. PubMed PMID: 33850773; PubMed Central PMCID: PMCPMC8039628.

124. Chakraborty M, Baldwin-Brown JG, Long AD, Emerson JJ. Contiguous and accurate de novo assembly of metazoan genomes with modest long read coverage. Nucleic Acids Res. 2016;44(19):e147. Epub 2016/11/02. doi: 10.1093/nar/gkw654. PubMed PMID: 27458204; PubMed Central PMCID: PMCPMC5100563.

125. Chin CS, Alexander DH, Marks P, Klammer AA, Drake J, Heiner C, et al. Nonhybrid, finished microbial genome assemblies from long-read SMRT sequencing data. Nat Methods. 2013;10(6):563-9. Epub 2013/05/07. doi: 10.1038/nmeth.2474. PubMed PMID: 23644548. integrated tool for comprehensive microbial variant detection and genome assembly improvement. PLoS One. 2014;9(11):e112963. Epub 2014/11/20. doi: 
10.1371/journal.pone.0112963. PubMed PMID: 25409509; PubMed Central PMCID: PMCPMC4237348.

127. Ruan J, Li H. Fast and accurate long-read assembly with wtdbg2. Nat Methods. 2019. Epub 2019/12/11. doi: 10.1038/s41592-019-0669-3. PubMed PMID: 31819265.

128. Kolmogorov M, Yuan J, Lin Y, Pevzner PA. Assembly of long, error-prone reads using repeat graphs. Nat Biotechnol. 2019;37(5):540-6. Epub 2019/04/03. doi: 10.1038/s41587-0190072-8. PubMed PMID: 30936562.

129. Altschul SF, Gish W, Miller W, Myers EW, Lipman DJ. Basic local alignment search tool. J Mol Biol. 1990;215(3):403-10. Epub 1990/10/05. doi: 10.1016/S0022-2836(05)80360-2. PubMed PMID: 2231712.

130. Laetsch D, Blaxter M. BlobTools: Interrogation of genome assemblies [version 1; peer review: 2 approved with reservations]. F1000Research. 2017;6(1287). doi:

10.12688/f1000research.12232.1. 131. Li H, Durbin R. Fast and accurate long-read alignment with Burrows-Wheeler transform. Bioinformatics. 2010;26(5):589-95. Epub 2010/01/19. doi: 10.1093/bioinformatics/btp698. PubMed PMID: 20080505; PubMed Central PMCID: PMCPMC2828108.

132. Li H, Handsaker B, Wysoker A, Fennell T, Ruan J, Homer N, et al. The Sequence Alignment/Map format and SAMtools. Bioinformatics. 2009;25(16):2078-9. Epub 2009/06/10. doi: 10.1093/bioinformatics/btp352. PubMed PMID: 19505943; PubMed Central PMCID: PMCPMC2723002.

133. Nouhaud P. Long-read based assembly and annotation of a <em $>$ Drosophila simulans</em> genome. bioRxiv. 2018:425710. doi: 10.1101/425710.

134. Gordon SP, Tseng E, Salamov A, Zhang J, Meng X, Zhao Z, et al. Widespread Polycistronic Transcripts in Fungi Revealed by Single-Molecule mRNA Sequencing. PLoS One. 2015;10(7):e0132628. Epub 2015/07/16. doi: 10.1371/journal.pone.0132628. PubMed PMID: 26177194; PubMed Central PMCID: PMCPMC4503453.

135. Holt C, Yandell M. MAKER2: an annotation pipeline and genome-database management tool for second-generation genome projects. BMC Bioinformatics. 2011;12:491. Epub 2011/12/24. doi: 10.1186/1471-2105-12-491. PubMed PMID: 22192575; PubMed Central PMCID: PMCPMC3280279.

136. Pertea M, Pertea GM, Antonescu CM, Chang TC, Mendell JT, Salzberg SL. StringTie enables improved reconstruction of a transcriptome from RNA-seq reads. Nat Biotechnol. 2015;33(3):290-5. Epub 2015/02/19. doi: 10.1038/nbt.3122. PubMed PMID: 25690850; PubMed Central PMCID: PMCPMC4643835.

137. Smit A, Hubley R, Green P. RepeatMasker 2013. Available from: http://www.repeatmasker.org.

138. Chang $\mathrm{CH}$, Chavan A, Palladino J, Wei X, Martins NMC, Santinello B, et al. Islands of retroelements are major components of Drosophila centromeres. PLoS Biol.

2019;17(5):e3000241. Epub 2019/05/16. doi: 10.1371/journal.pbio.3000241. PubMed PMID: 31086362; PubMed Central PMCID: PMCPMC6516634.

139. Bailly-Bechet M, Haudry A, Lerat E. "One code to find them all": a perl tool to conveniently parse RepeatMasker output files. Mobile DNA. 2014;5(1):13. doi: 10.1186/17598753-5-13.

140. Benson G. Tandem repeats finder: a program to analyze DNA sequences. Nucleic Acids Res. 1999;27(2):573-80. Epub 1998/12/24. PubMed PMID: 9862982; PubMed Central PMCID: PMCPMC148217. 2012;9(4):357-9. Epub 2012/03/06. doi: 10.1038/nmeth.1923. PubMed PMID: 22388286; PubMed Central PMCID: PMCPMC3322381. aware quantification of transcript expression. Nat Methods. 2017;14(4):417-9. Epub 2017/03/07. 
doi: 10.1038/nmeth.4197. PubMed PMID: 28263959; PubMed Central PMCID: PMCPMC5600148.

143. Langmead B. Aligning short sequencing reads with Bowtie. Current protocols in bioinformatics / editoral board, Andreas D Baxevanis [et al]. 2010;Chapter 11:Unit 11 7. Epub 2010/12/15. doi: 10.1002/0471250953.bi1107s32. PubMed PMID: 21154709; PubMed Central PMCID: PMCPMC3010897.

144. Mose LE, Perou CM, Parker JS. Improved indel detection in DNA and RNA via realignment with ABRA2. Bioinformatics. 2019;35(17):2966-73. Epub 2019/01/17. doi: 10.1093/bioinformatics/btz033. PubMed PMID: 30649250; PubMed Central PMCID: PMCPMC6735753.

145. Huang W, Li L, Myers JR, Marth GT. ART: a next-generation sequencing read simulator. Bioinformatics. 2012;28(4):593-4. Epub 2011/12/27. doi: 10.1093/bioinformatics/btr708. PubMed PMID: 22199392; PubMed Central PMCID: PMCPMC3278762.

146. Larracuente AM, Ferree PM. Simple method for fluorescence DNA in situ hybridization to squashed chromosomes. JoVE. 2015;95:e52288. doi: doi:10.3791/52288. 147. Pimpinelli S, Bonaccorsi S, Fanti L, Gatti M. Immunostaining of mitotic chromosomes from Drosophila larval brain. Cold Spring Harbor protocols. 2011;2011(9). doi: 10.1101/pdb.prot065524. PubMed PMID: 21880821. 148. Kearse M, Moir R, Wilson A, Stones-Havas S, Cheung M, Sturrock S, et al. Geneious Basic: an integrated and extendable desktop software platform for the organization and analysis of sequence data. Bioinformatics. 2012;28(12):1647-9. Epub 2012/05/01. doi:

10.1093/bioinformatics/bts199. PubMed PMID: 22543367; PubMed Central PMCID: PMCPMC3371832.

149. Nguyen LT, Schmidt HA, von Haeseler A, Minh BQ. IQ-TREE: a fast and effective stochastic algorithm for estimating maximum-likelihood phylogenies. Mol Biol Evol. 2015;32(1):268-74. Epub 2014/11/06. doi: 10.1093/molbev/msu300. PubMed PMID: 25371430; PubMed Central PMCID: PMCPMC4271533.

150. Hoang DT, Chernomor O, von Haeseler A, Minh BQ, Vinh LS. UFBoot2: Improving the Ultrafast Bootstrap Approximation. Mol Biol Evol. 2018;35(2):518-22. Epub 2017/10/28. doi: 10.1093/molbev/msx281. PubMed PMID: 29077904; PubMed Central PMCID: PMCPMC5850222.

151. Kumar S, Stecher G, Li M, Knyaz C, Tamura K. MEGA X: Molecular Evolutionary Genetics Analysis across Computing Platforms. Mol Biol Evol. 2018;35(6):1547-9. Epub 2018/05/04. doi: 10.1093/molbev/msy096. PubMed PMID: 29722887; PubMed Central PMCID: PMCPMC5967553.

152. Stecher G, Tamura K, Kumar S. Molecular Evolutionary Genetics Analysis (MEGA) for macOS. Mol Biol Evol. 2020. Epub 2020/01/07. doi: 10.1093/molbev/msz312. PubMed PMID: 31904846.

153. Paradis E, Claude J, Strimmer K. APE: Analyses of Phylogenetics and Evolution in R language. Bioinformatics. 2004;20(2):289-90. Epub 2004/01/22. PubMed PMID: 14734327. 154. Thornton K. Libsequence: a C++ class library for evolutionary genetic analysis. Bioinformatics. 2003;19(17):2325-7. Epub 2003/11/25. PubMed PMID: 14630667.

155. Hudson RR. Estimating the recombination parameter of a finite population model without selection. Genetical research. 1987;50(3):245-50. PubMed PMID: 3443297.

156. Hudson RR, Kaplan NL. Statistical properties of the number of recombination events in the history of a sample of DNA sequences. Genetics. 1985;111(1):147-64. Epub 1985/09/01. PubMed PMID: 4029609; PubMed Central PMCID: PMCPMC1202594. genomes, a new PANTHER GO-slim and improvements in enrichment analysis tools. Nucleic PMID: 30407594; PubMed Central PMCID: PMCPMC6323939. 


\section{Supplementary text}

\section{Validation of variants in Y-linked gene families}

1310 We mapped Illumina reads from male genomic DNA and testis RNAseq to the

1311 reconstructed ancestral transcript sequences of each gene cluster (Lhk-1, Lhk-2,

1312 CK2ßtes-Y) to estimate the expression level of the different $Y$-linked copies. We first

1313 asked if the variants in these two gene families found in our assemblies can be

1314 consistently detected in Illumina reads from male genomes. We found that the

1315 abundance of derived variants in these two gene families in the DNA-seq data are

1316 highly correlated to the frequency of variants in our assemblies $(\mathrm{R}=0.89$ and 0.98 in $D$.

1317 mauritiana and $D$. simulans, respectively). For 559 variants in the $D$. simulans

1318 assembly, 33 of them (28 appear once and four appear twice) are missing from the

1319 DNA-seq data. For 446 variants in the D. mauritiana assembly, 43 of them (32 appear

1320 once and six appear twice) are missing from the DNA-seq data. Additionally, nine and

1321 eight inconsistent variants are located near (<100 bp) the start or end of transcripts in

1322 D. simulans and D. mauritiana, respectively. These regions at the edges of transcripts

1323 might have fewer Illumina reads coverage than more central regions.

1324 We compared the proportion of synonymous and nonsynonymous changes between

1325 copies with high and low expression using transcriptome data to infer selection

1326 pressures on different mutations (Fig S10; Table S21).

1327 To reduce the effect of sequencing errors and simplify the phylogenetic analyses on 1328 protein evolution rates, we first reconstructed the ancestral sequences of each gene 1329 cluster (Lhk-1, Lhk-2, CK2ßtes-Y, and 2 CK2ßtes-like; see Fig 5). The reconstructed 
1330 ancestral sequences should eliminate misassembled bases, which are typically

1331 singletons. We conducted branch-model and branch-site-model tests on the

1332 reconstructed ancestral sequence using PAML and inferred that both gene families

1333 experienced strong positive selection following their duplication to the $Y$ chromosome

1334 (from branch model; Tables S17 and S18, Fig 5). The high rate of protein evolution in

1335 the $\mathrm{Y}$-linked ampliconic genes suggests that, in addition to subfunctionalization or

1336 degeneration, they may also acquire new functions and adapt to being Y-linked. 


\section{Supplementary Figures}

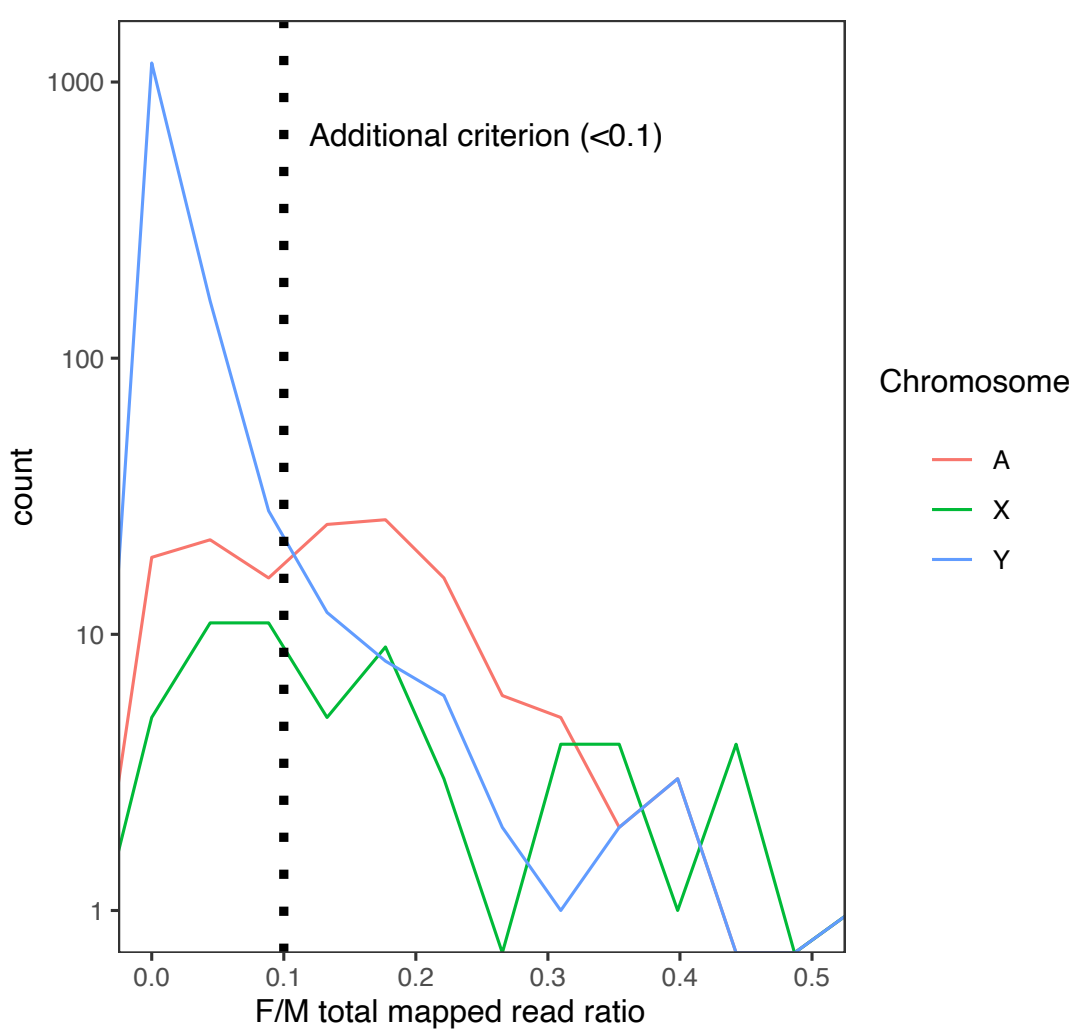

Fig S1. The distribution of female to male total mapped read ratio in each 10-kb window in $\boldsymbol{D}$. mauritiana. Many non-Y regions have median male-to-female coverage 0 in our $D$. mauritiana data. Therefore, we applied an additional criterion based on the female-to-male total mapped reads ratio $(<0.1)$ to reduce the false-positive rate.

\section{D. mauritiana}

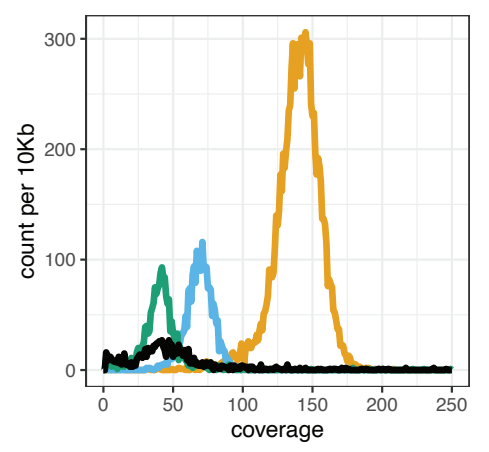

D. simulans

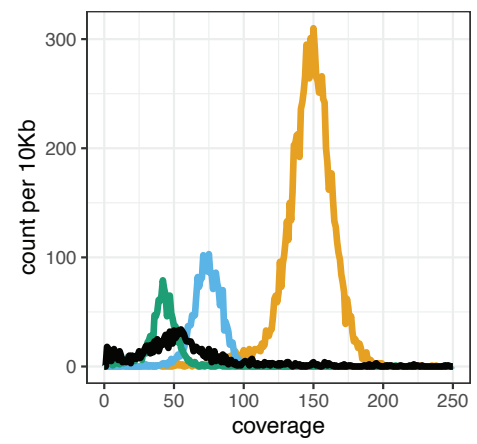

D. sechellia

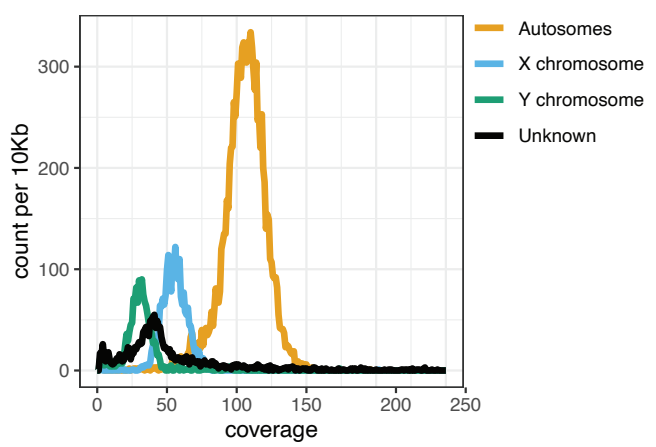

Fig S2. The low Pacbio coverage on the $Y$ chromosome in the $D$. simulans clade. We calculated the median coverage of Pacbio reads every $10-\mathrm{kb}$ and plotted the histogram of depth across genomes based on their chromosome location. 


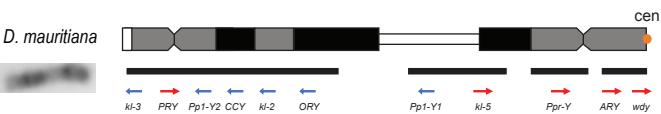

\begin{tabular}{l}
\hline AATAT \\
\hline AAGAG \\
\hline ATTAG \\
\hline AAAATG \\
\hline ATAGTACAG \\
\hline AAACAT \\
\hline AATTAAC \\
\hline PRPK
\end{tabular}
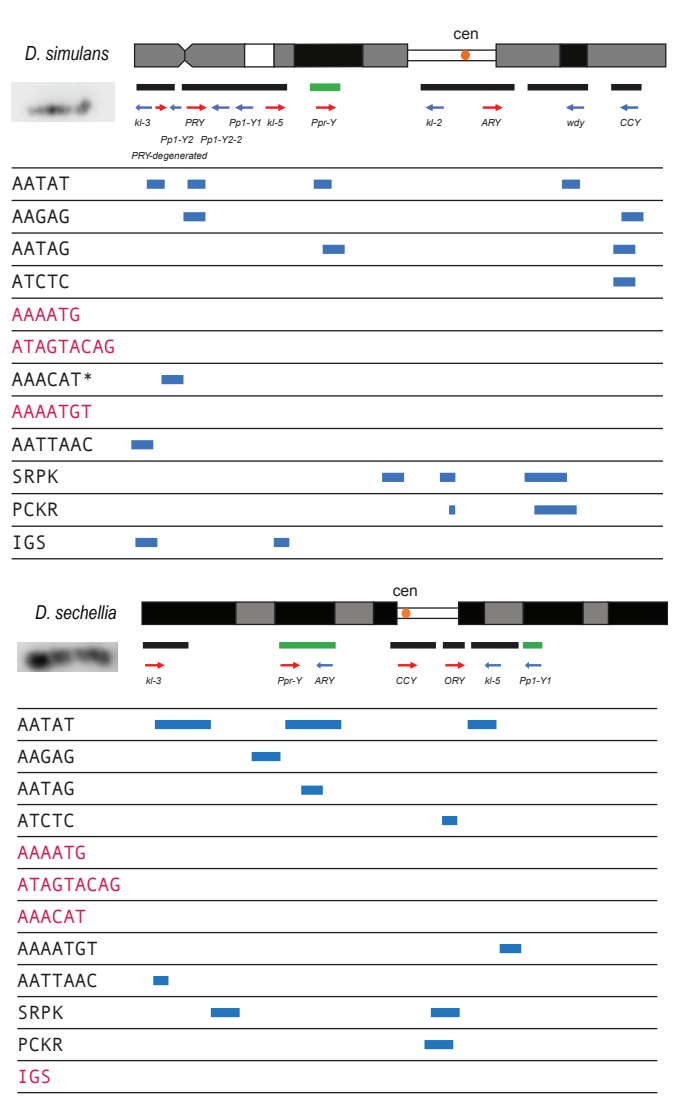
conserved genes on the $Y$ chromosome of the $D$. simulans clade. We used FISH as well as our assemblies to infer the cytological location of $Y$-linked sequences. The bars represent the location of scaffolds or contigs, and the green bars are scaffolds or contigs without known direction. The satellites in red are sequences we cannot detect on $\mathrm{Y}$ chromosomes using FISH. *Based on the repeat content from the Illumina data (Table S16), the AAACAT signal is probably from the AAACAAT tandem array, instead of AAACAT, in D. simulans. 

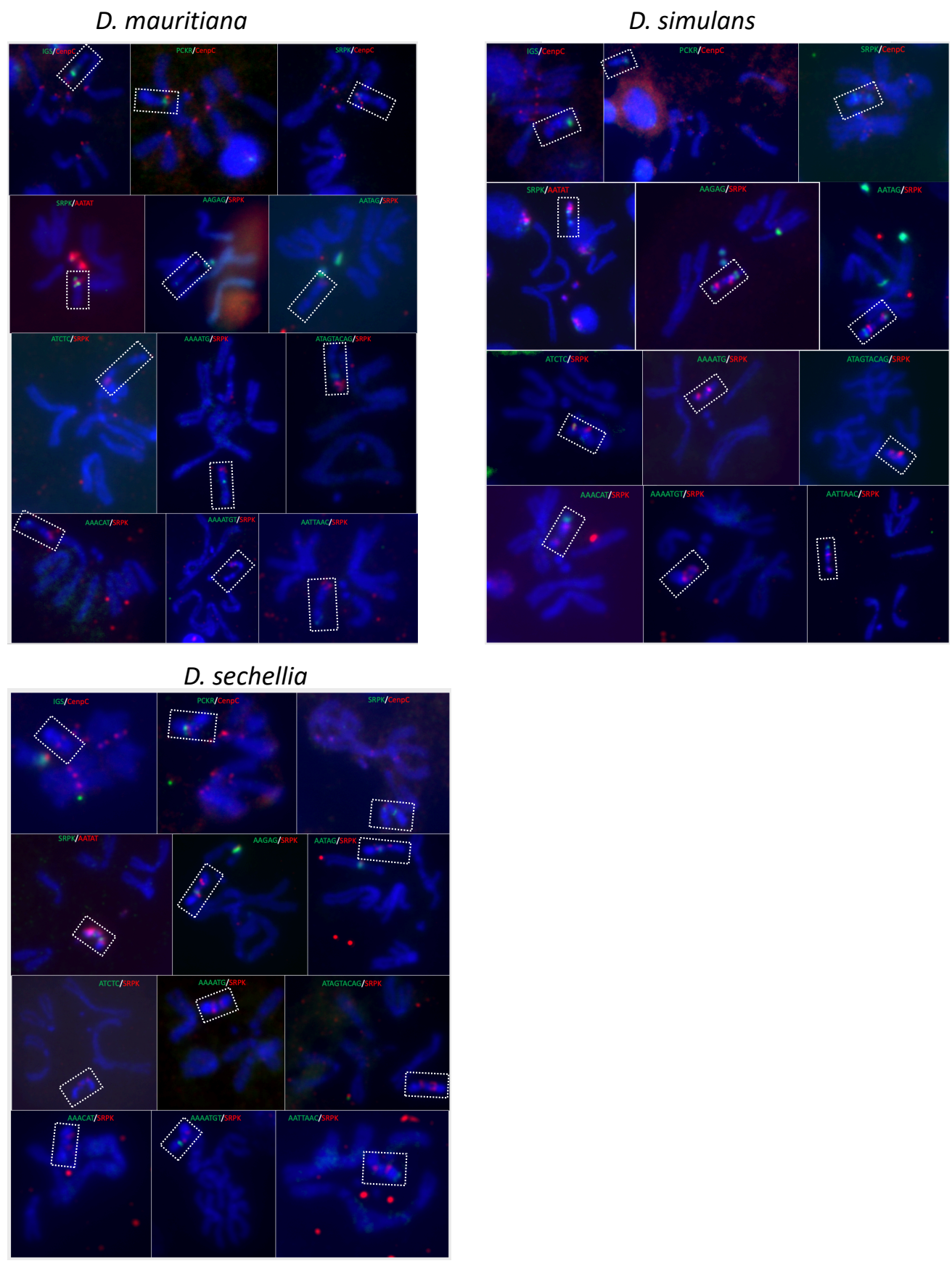
immunostaining. The colors on the figure represent the probes we used for the experiments. 

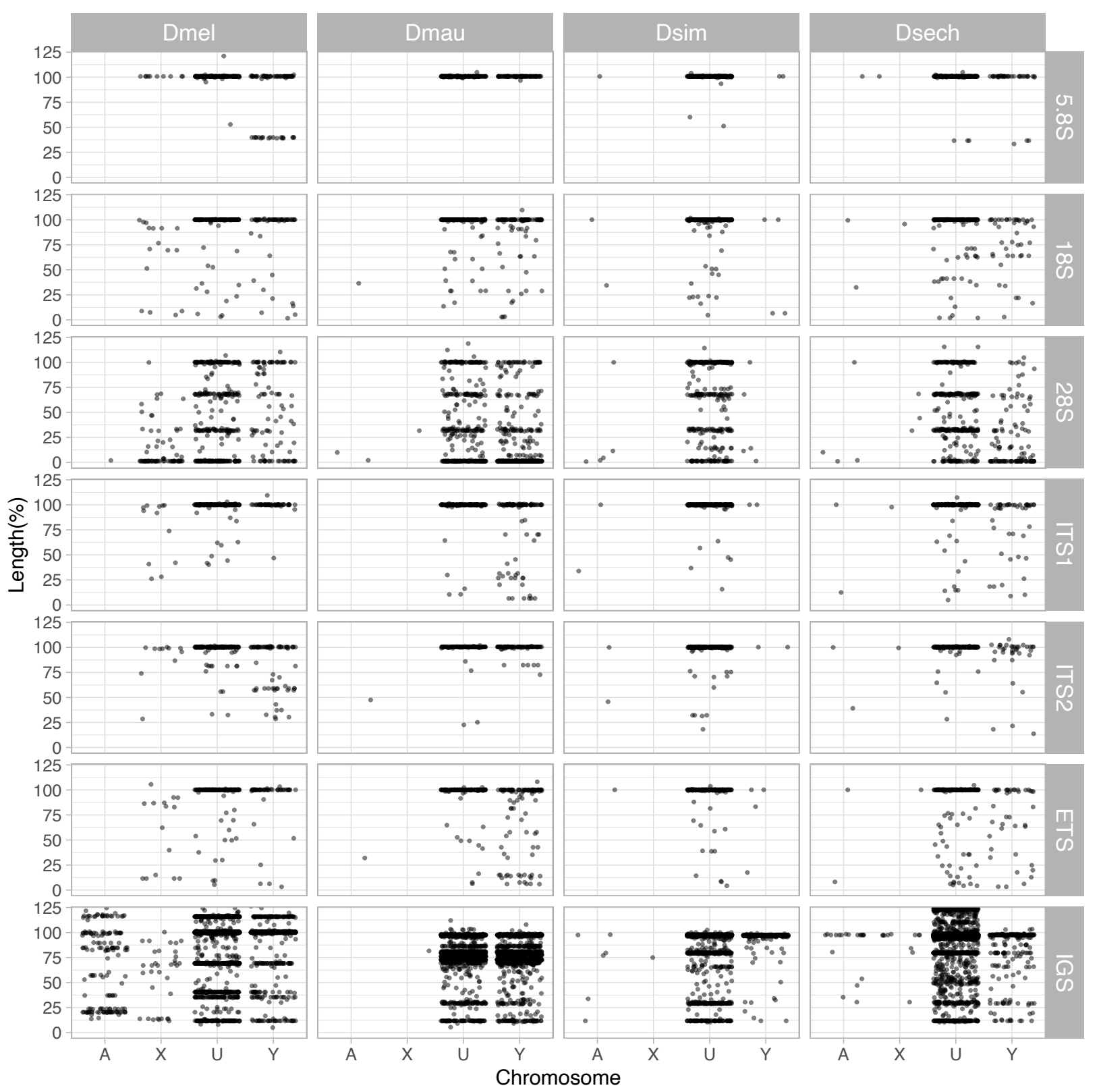

Fig S5. The length of rDNA elements across the chromosomes in D. melanogaster and the $D$. simulans clade.We surveyed the length of rDNA elements across chromosomes ( $A$ : autosomes, $X$ : $X$ chromosome, $U$ : unknown location and $Y: Y$ chromosome). The length of elements is normalized by the length of consensus from functional elements. 
bioRxiv preprint doi: https://doi.org/10.1101/2021.08.16.456461: this version posted August 16, 2021. The copyright holder for this preprint (which was not certified by peer review) is the author/funder, who has granted bioRxiv a license to display the preprint in perpetuity. It is made available under aCC-BY 4.0 International license.
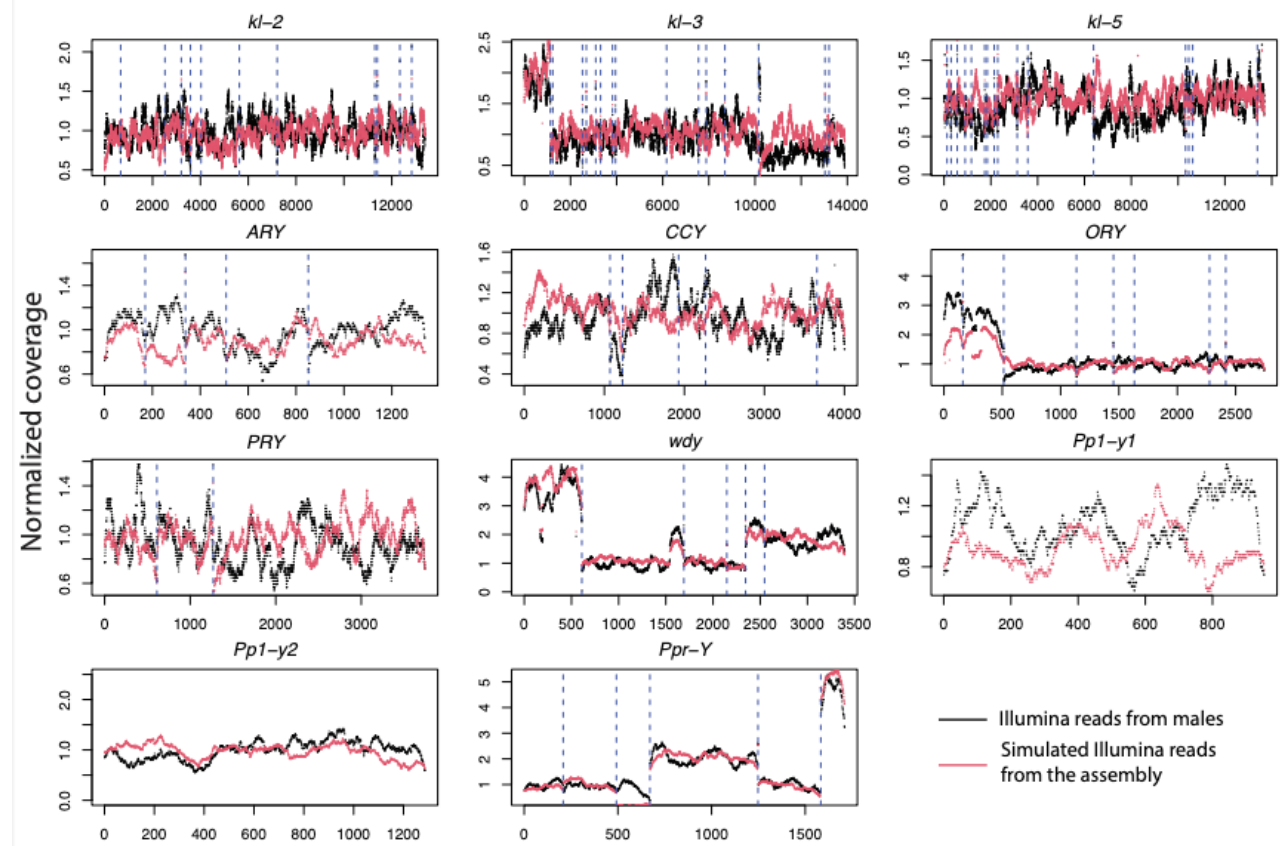

\section{Drosophila mauritiana}
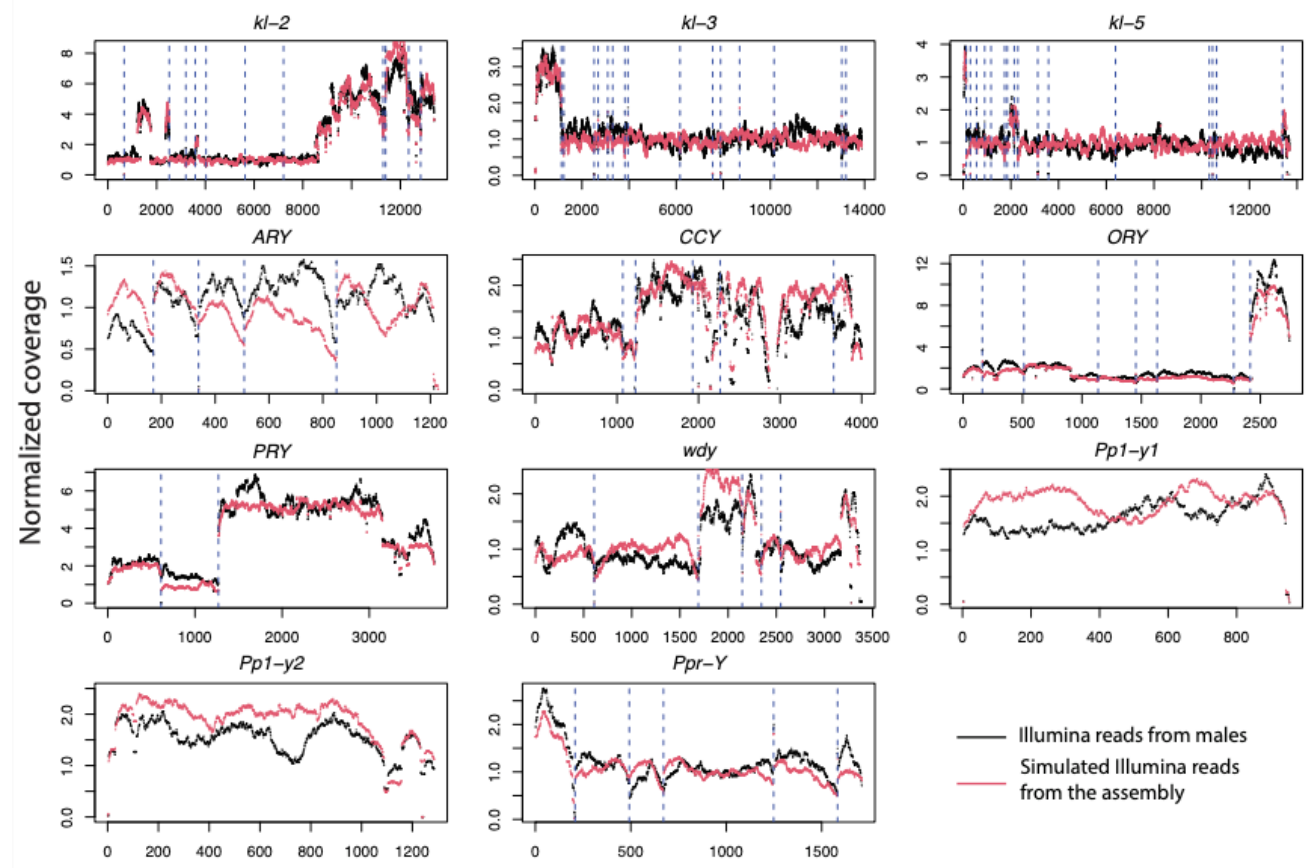

Illumina reads from males
Simulated Illumina reads

from the assembly 


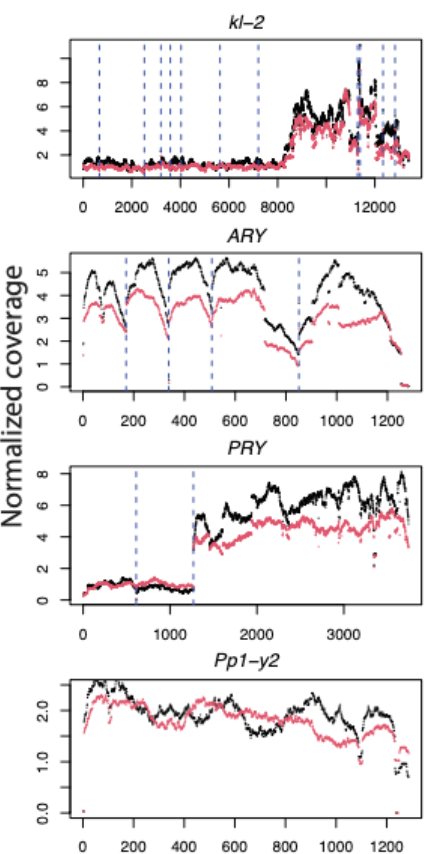

\section{Drosophila simulans}
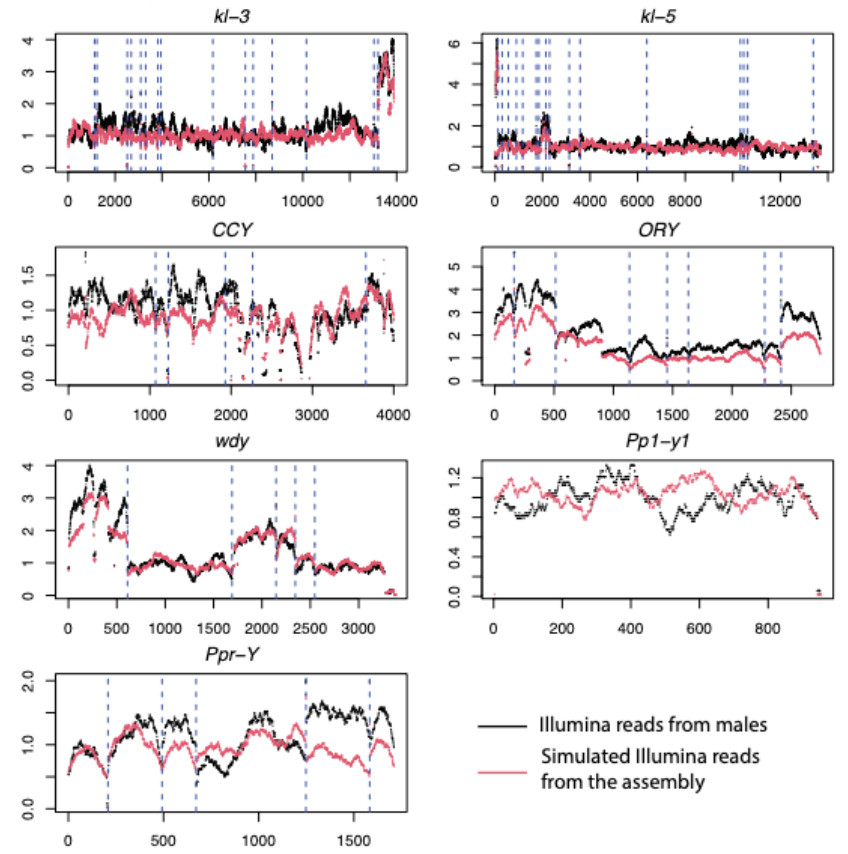

\section{Drosophila sechellia}
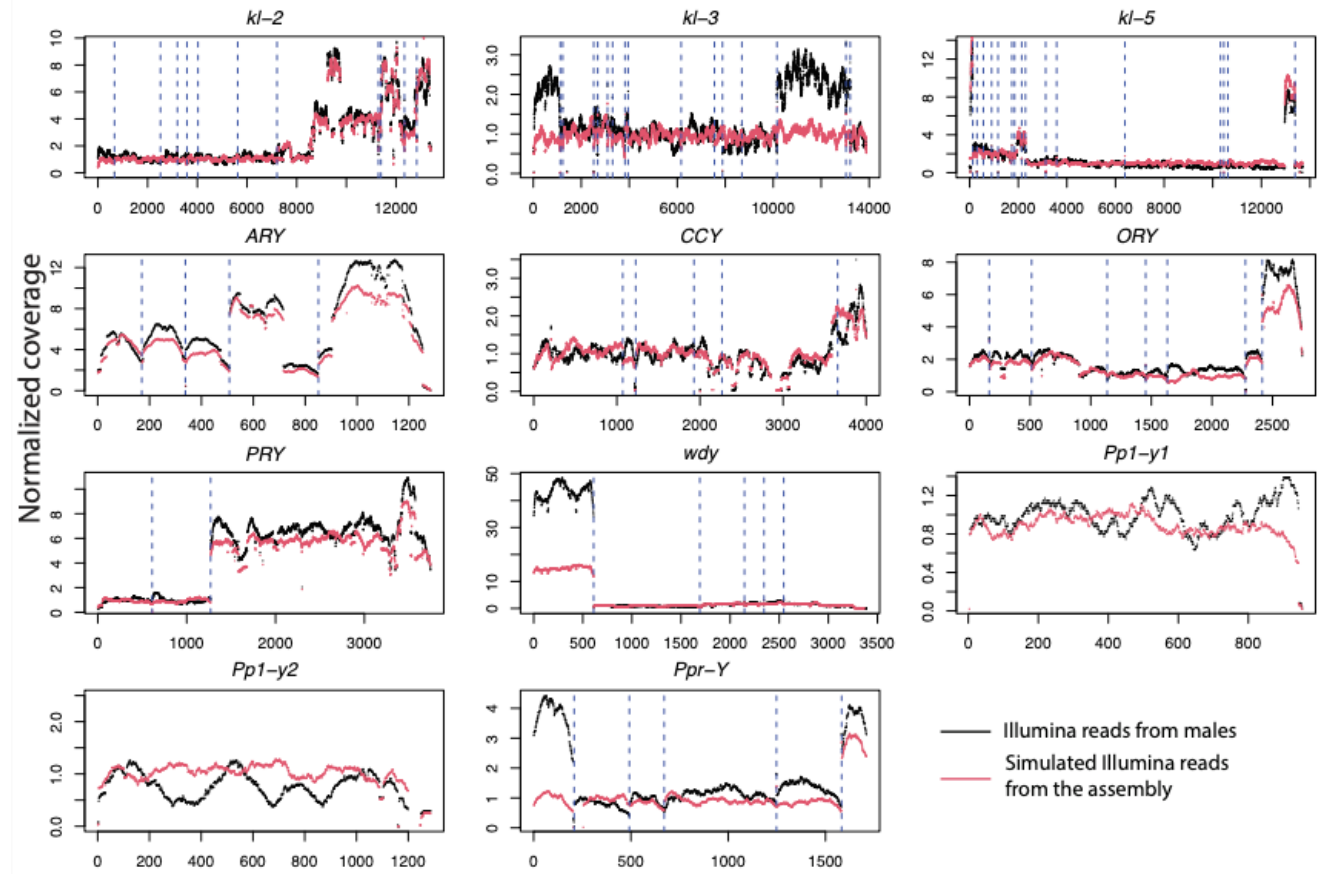

— Illumina reads from males Simulated Illumina reads from the assembly

Fig S6. The copy number of male Illumina DNA-seq reads in 11 canonical Y-linked genes. To confirm the copy number of $Y$-linked genes across species in our assembly, we mapped the Illumina reads from males to a single of $D$. melanogaster $Y$-linked transcripts and estimated the copy number based on their coverage (black lines). For the comparison, we also simulated Illumina reads from our assemblies and mapped them to the same reference to estimate their copy number (red lines). The dotted lines separate each exon. 
bioRxiv preprint doi: https://doi.org/10.1101/2021.08.16.456461; this version posted August 16, 2021. The copyright holder for this preprint (which was not certified by peer review) is the author/funder, who has granted bioRxiv a license to display the preprint in perpetuity. It is made available under aCC-BY 4.0 International license.
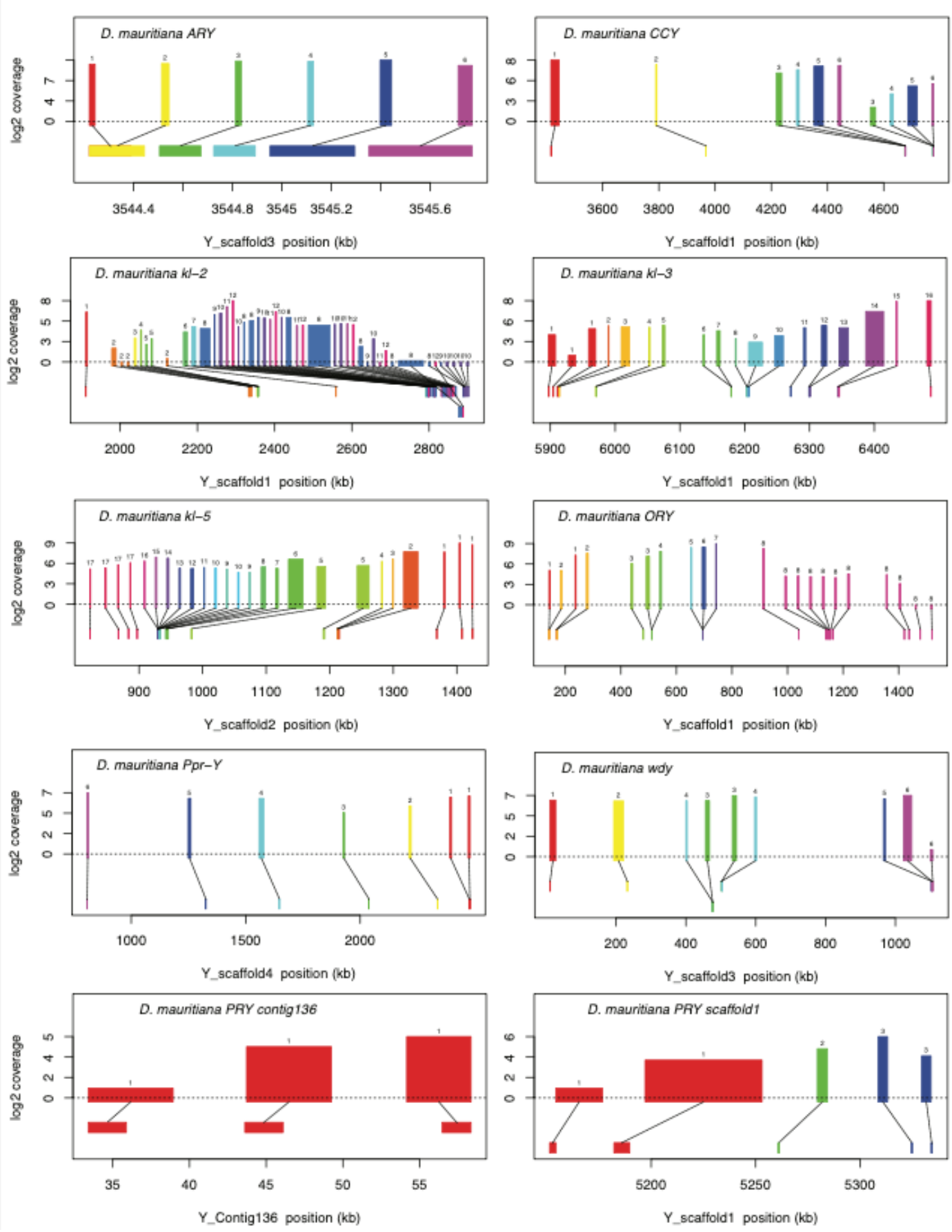
bioRxiv preprint doi: https://doi.org/10.1101/2021.08.16.456461; this version posted August 16, 2021. The copyright holder for this preprint (which was not certified by peer review) is the author/funder, who has granted bioRxiv a license to display the preprint in perpetuity. It is made available under aCC-BY 4.0 International license.
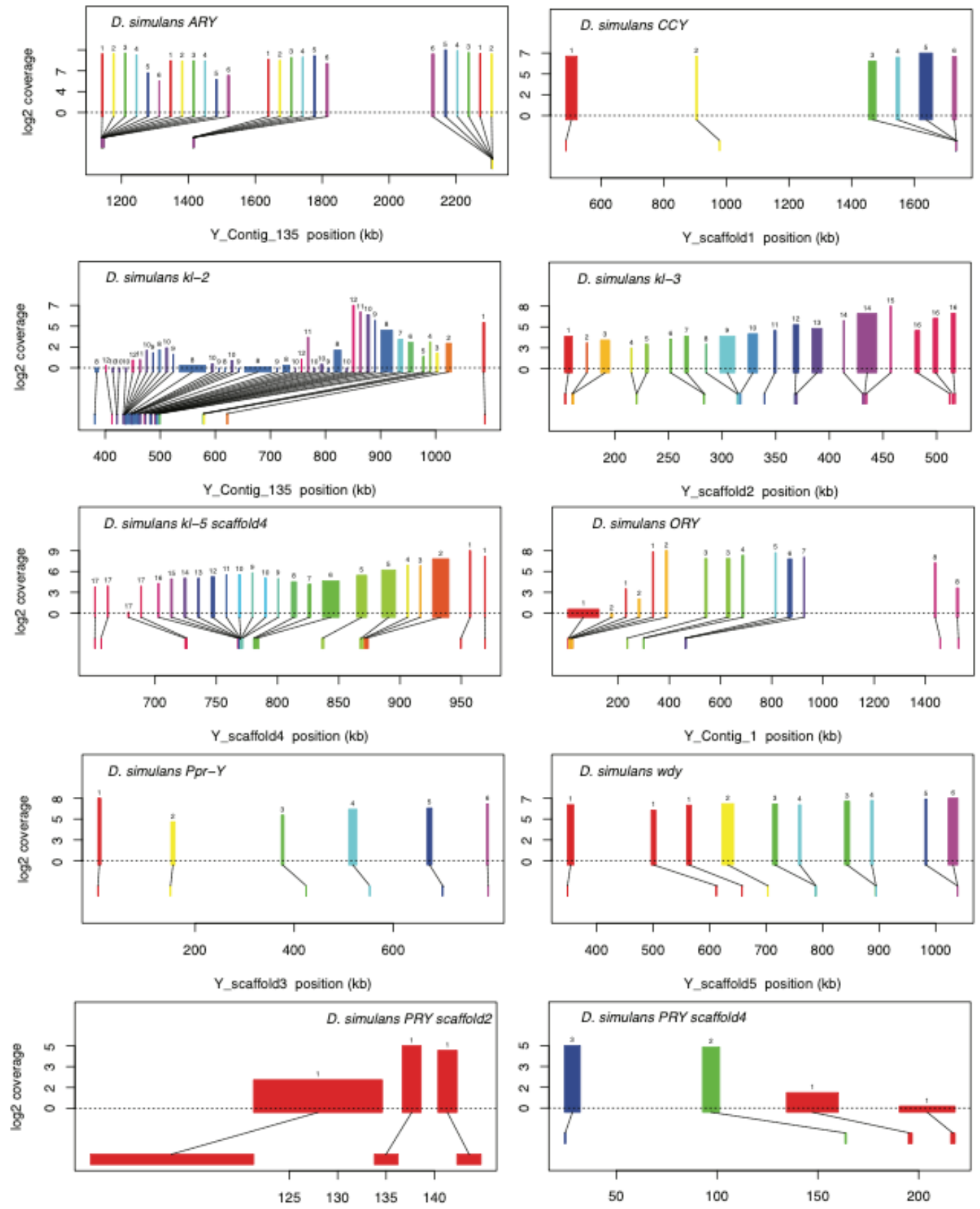

Y_scaftiold2 position (kb)

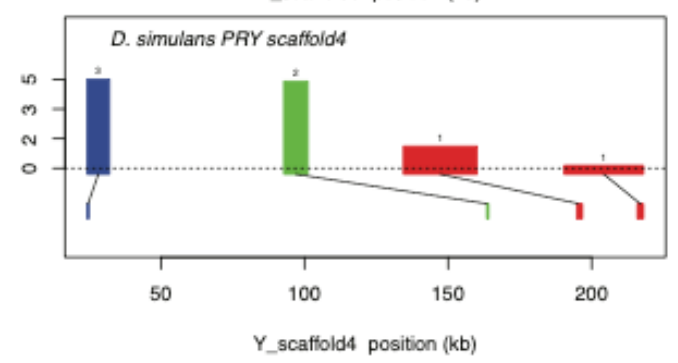



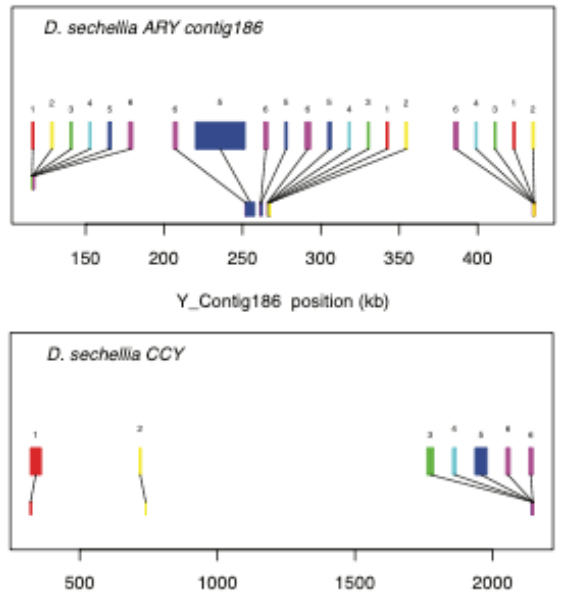

Y_scaffold1 position (kb)
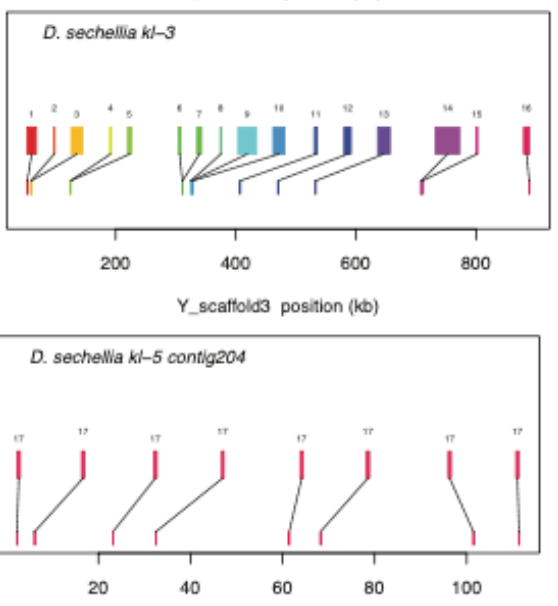

Y_Contig204 position (kb)

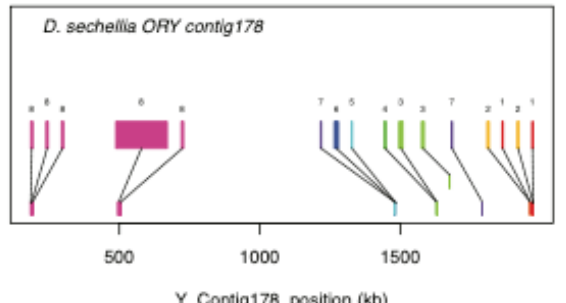

Contig178 position (kb)

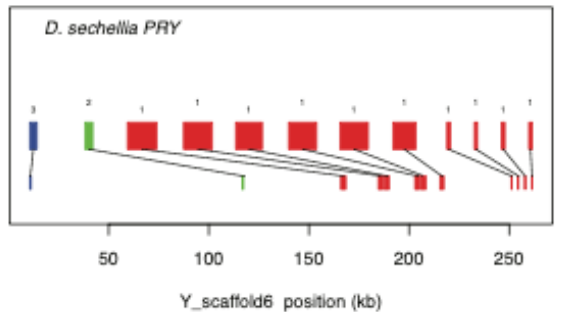

Y_scaftold6 position $(\mathrm{kb})$
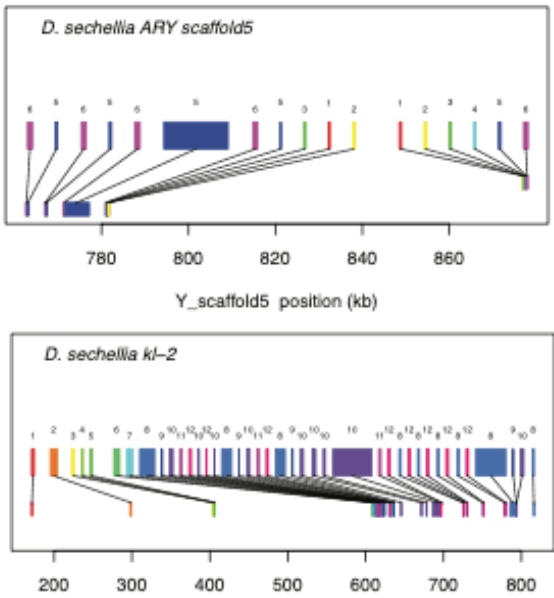

Y_scaffold2 position $(\mathrm{kb})$
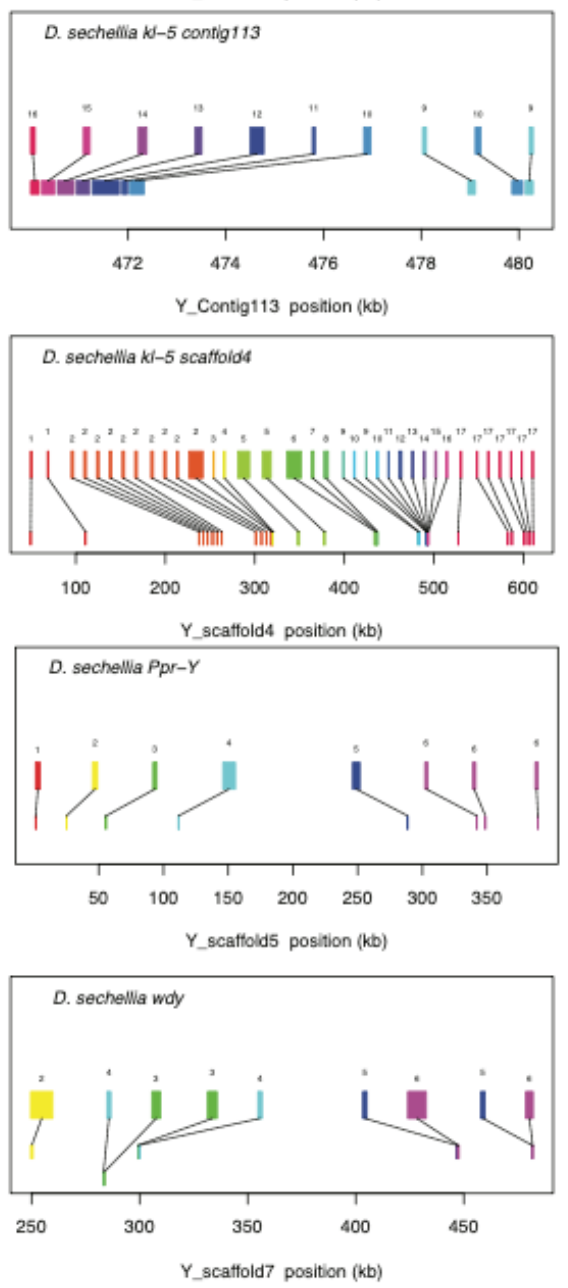

Fig S7. Gene structure of 11 conserved $Y$-linked genes inferred from assemblies and RNA-seq data. Upper bars indicate exons that are colored and numbered, with their height indicating average read depth from sequenced testes RNA ( $D$. simulans and $D$. mauritiana only). Lower bars indicate exon positions on the assembly and position on 1393 the Y-axis indicates coding strand. 


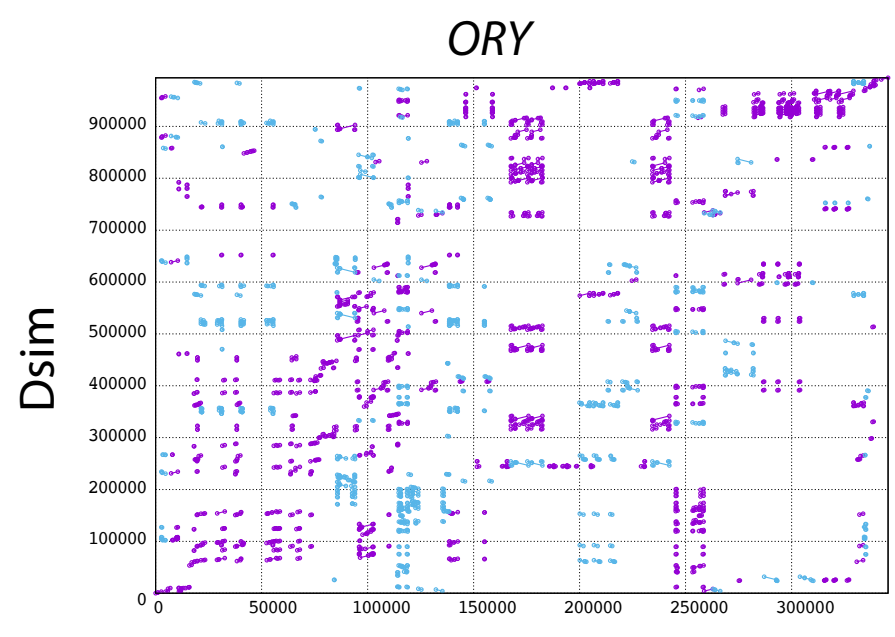

\section{Dmau}
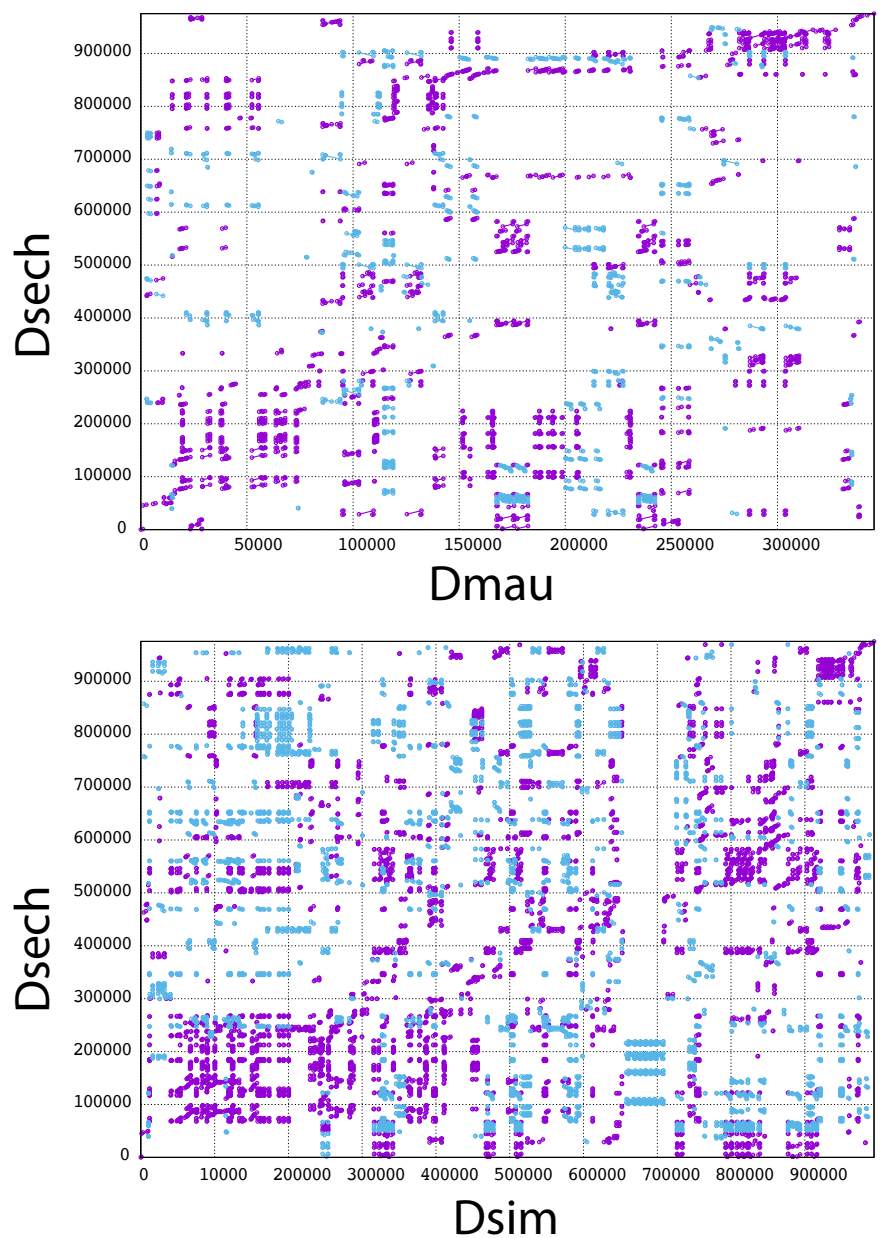
represent forward matches, and blue lines and dots represent reverse matches. 


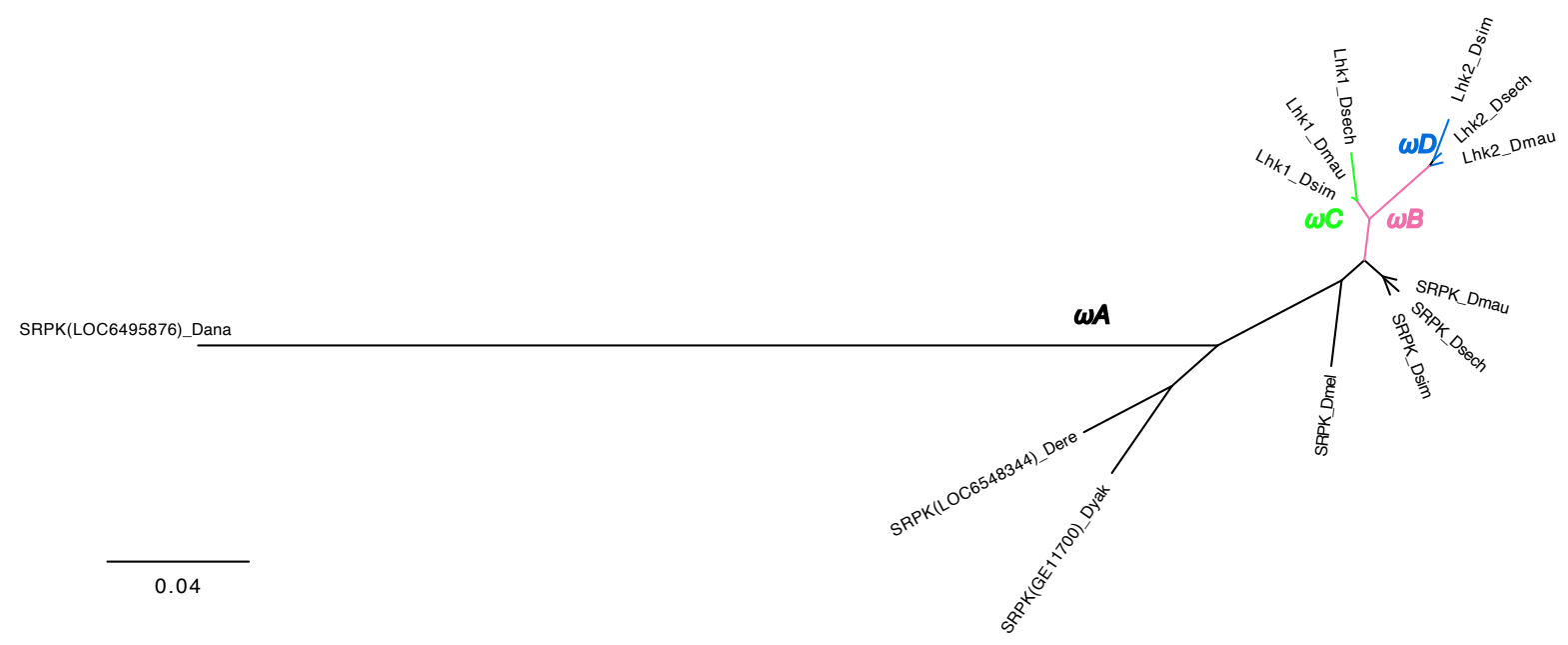

Fig S9. The phylogeny of Lhk used in PAML analyses. We marked the branches used in branch-model and branch-site model tests. We did all comparisons using the branch with different colors in likelihood-ratio tests. Please see the detailed results in Table S17. 


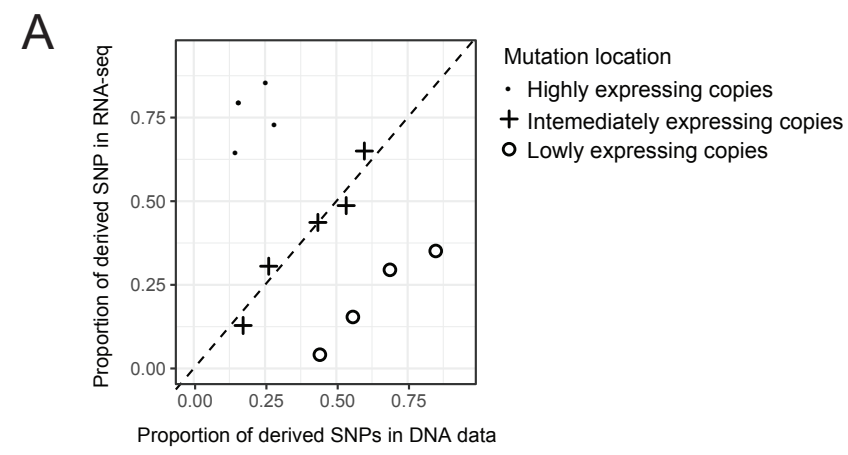

B

D. mauritiana
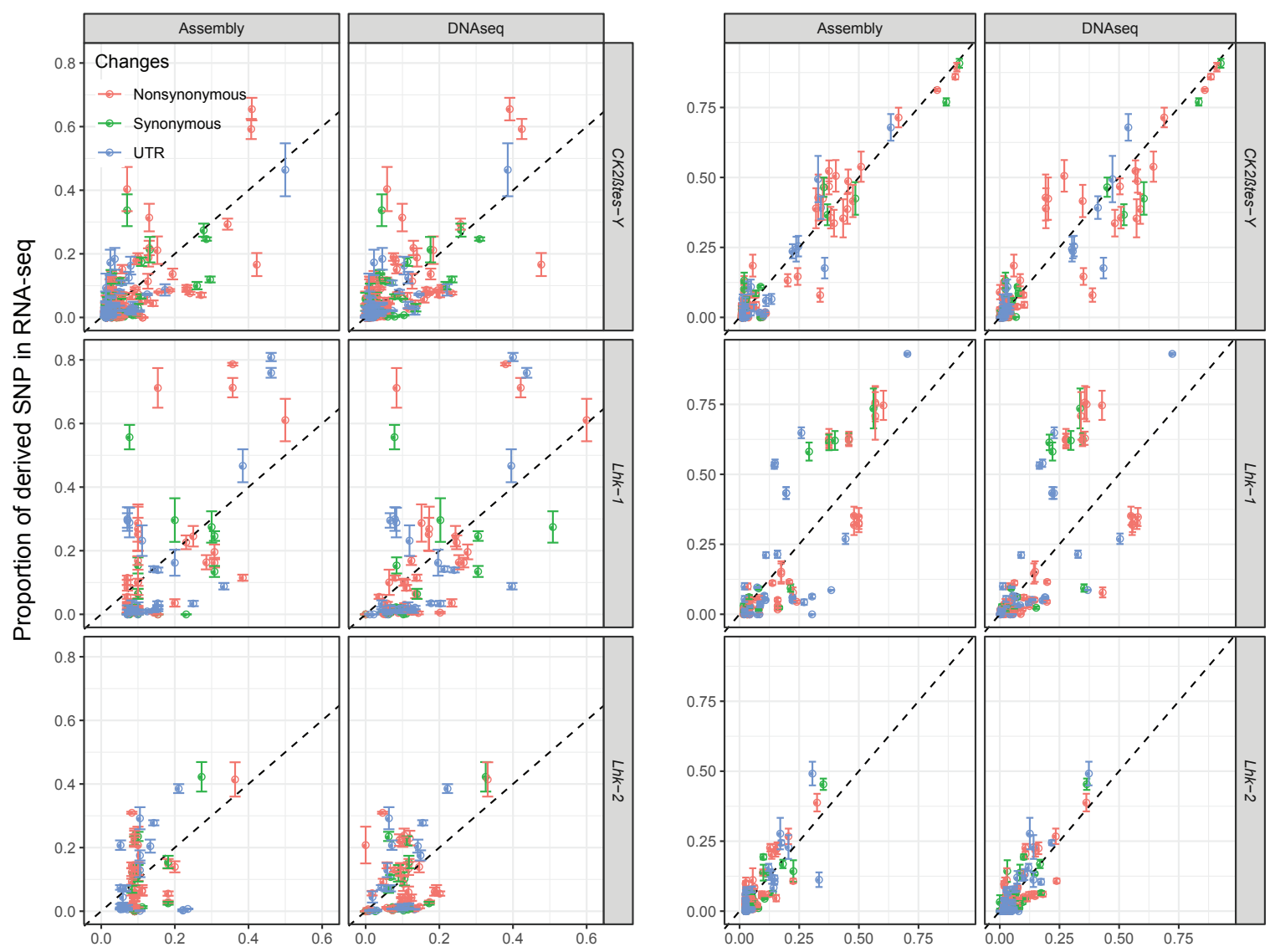

Proportion of derived SNPs in DNA data

S10. The expression of different copies from Lhk and CK2ßtes-Y gene families.

(A) We quantify the frequency of each derived SNP within the genome using DNA-seq and the expression level of each allele using RNA-seq. We cataloged each SNP as synonymous, nonsynonymous or UTR. (B) We found that across three Y-linked gene families, only highly expressed Lhk-1 copies have fewer nonsynonymous mutations than lowly expressed copies in $D$. simulans, consistent with purifying selection (Table $\mathrm{S} 12$ and S21; Chi-square test's $\mathrm{P}=0.01$ ). We did not detect other significant changes in other comparisons (Table S12 and S21; Chi-square test's P > 0.01). 


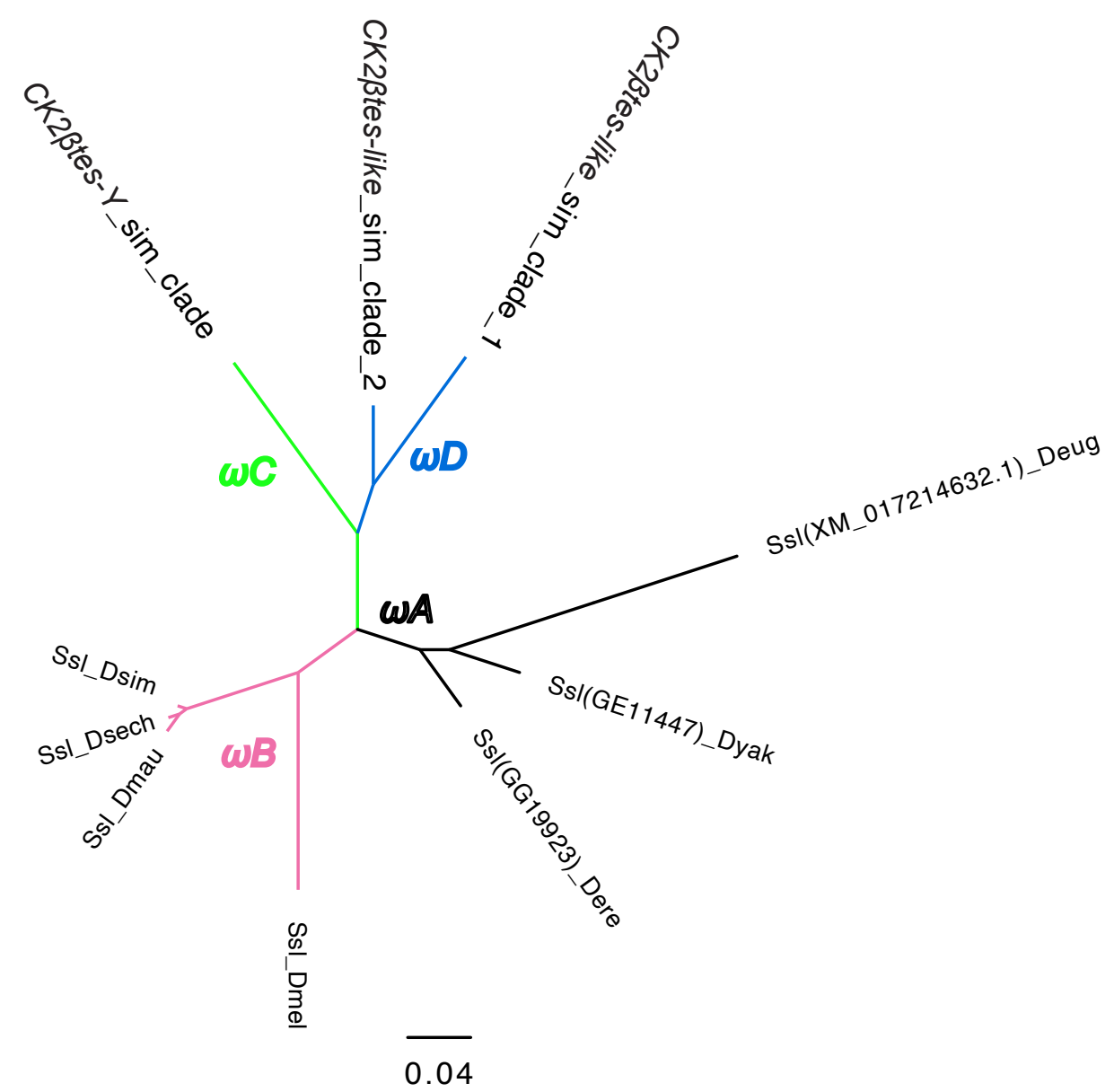

S11. The phylogeny of CK2ßtes-Y used in PAML analyses. We marked the

Fig branches used in branch-model and branch-site model tests. We did all comparisons using the branch with different colors in likelihood-ratio tests. Please see the detailed results in Table S18. 


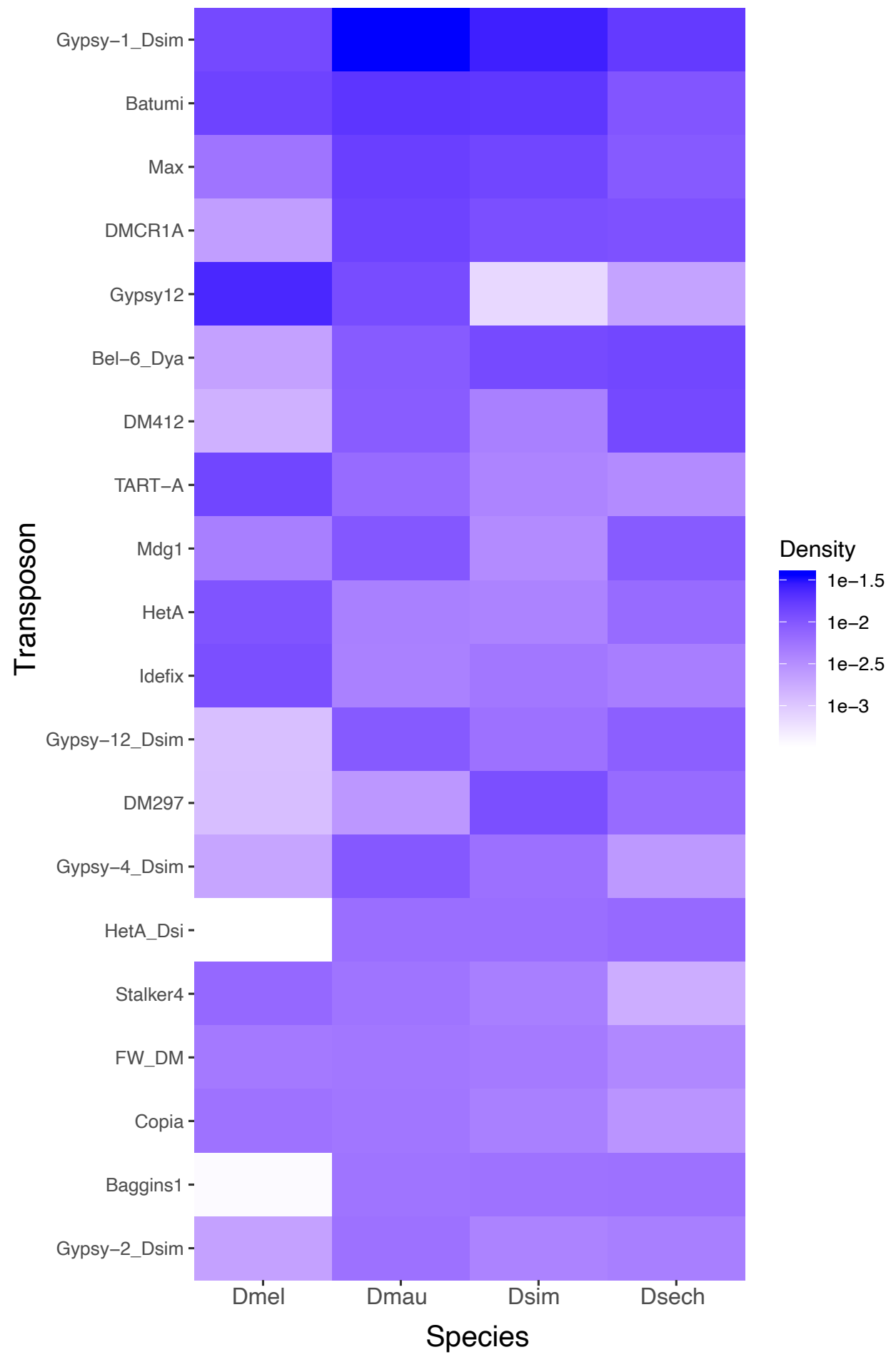

\section{Fig S12. The abundance of repetitive elements on $Y$ chromosomes of $D$.} melanogaster and the $\boldsymbol{D}$. simulans clade species. We plotted the density of 20 most enriched (by total occupying sequences) repetitive elements on $Y$ chromosomes across four species. The colors represent the proportion of repetitive sequences in all assembled Y-linked sequences. 


\section{Fold difference of TE abundance Other chromosomes}

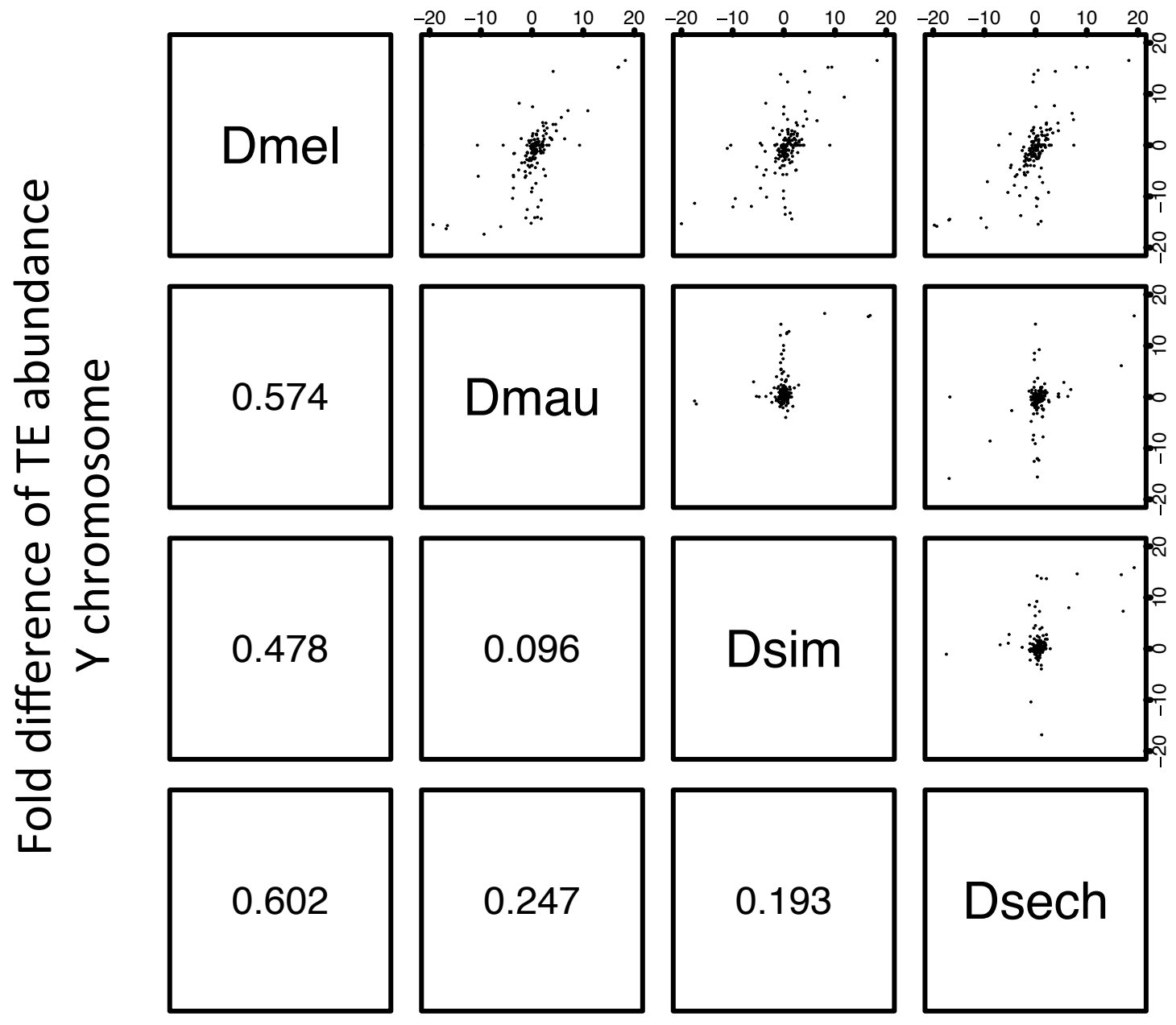

Fig S13. The correlation of TE abundance between $Y$ chromosomes and other chromosomes of $\boldsymbol{D}$. melanogaster and the $\boldsymbol{D}$. simulans clade. We calculated the fold changes of TE occupying sites (bp) between species by chromosomes. Each point from the figures above the diagonal represents the changes of a TE element on the $Y$ chromosome and the other (non-Y) chromosomes. The number below the diagonal shows Spearman's rank correlation coefficient for each comparison. 

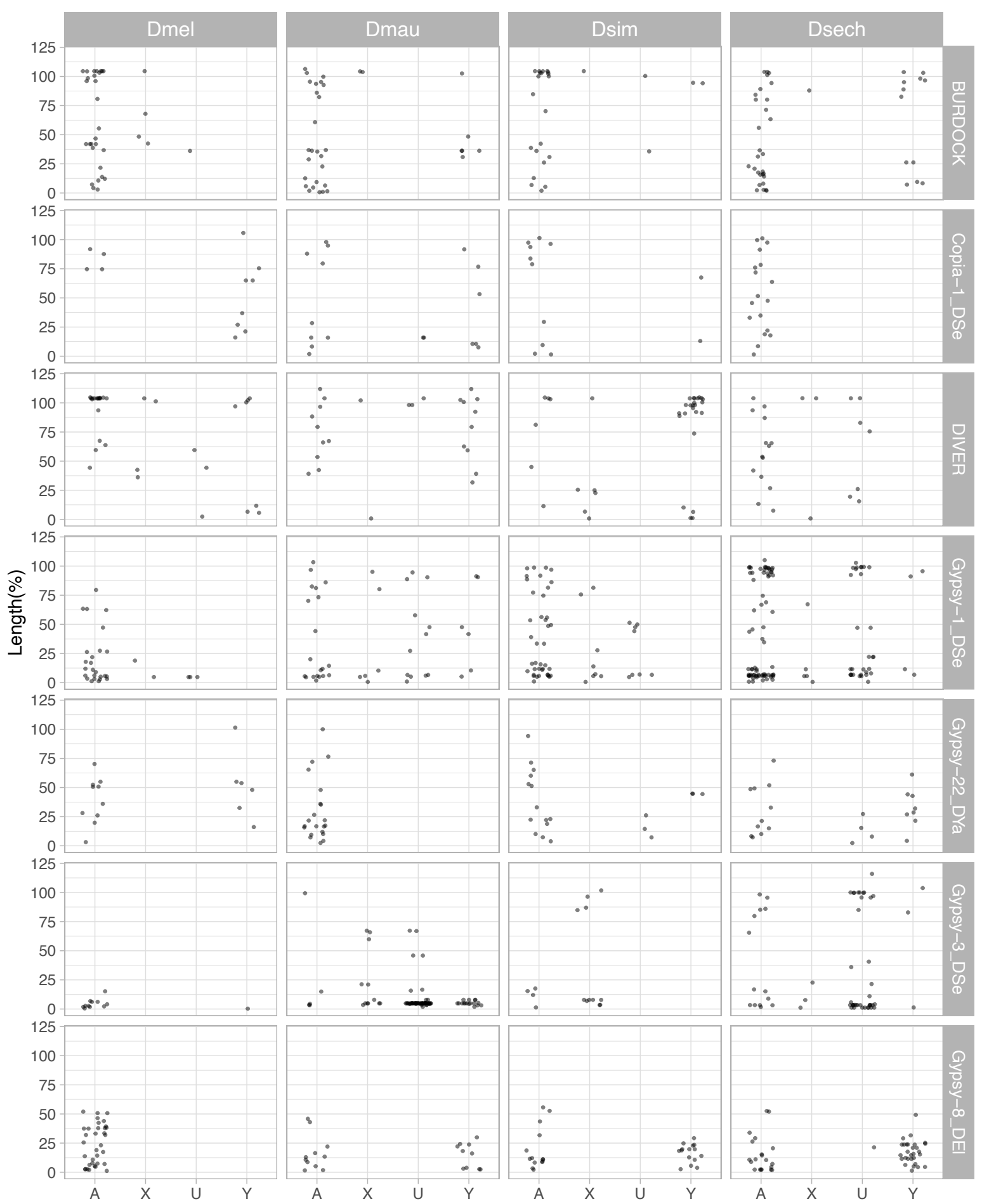

Chromosome
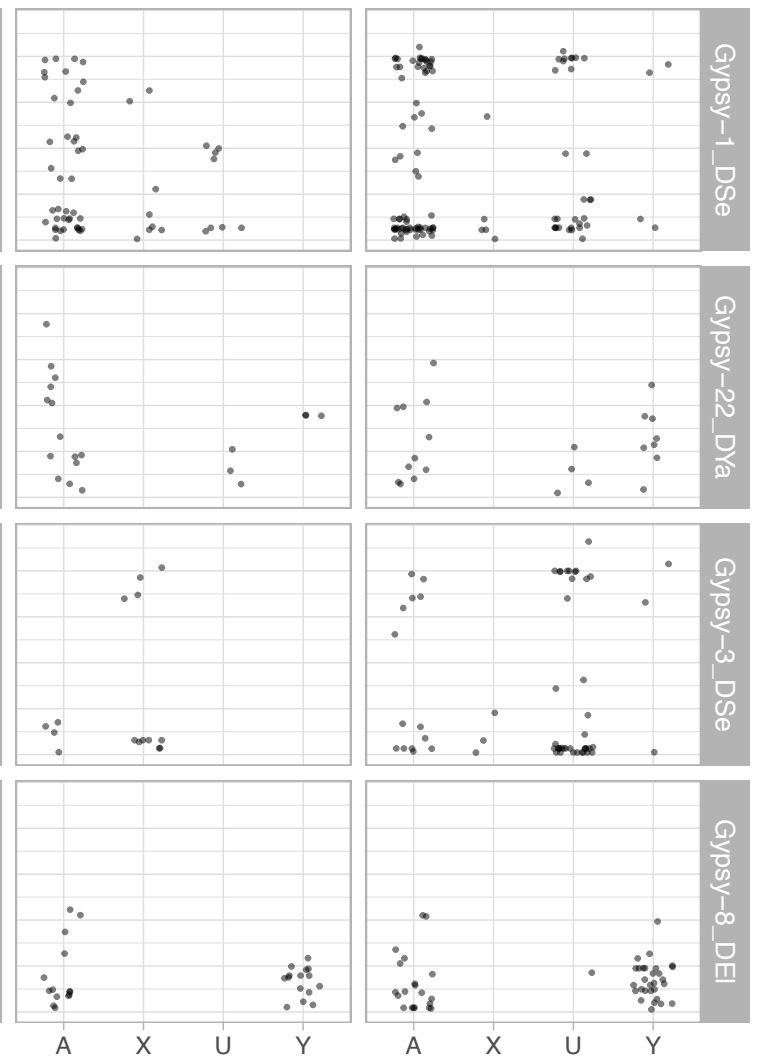

Fig S14. The length of LTR retrotransposons between $Y$ chromosomes and other chromosomes of $D$. melanogaster and the $D$. simulans clade. We surveyed the length of LTR retrotransposons across chromosomes (A: autosomes, $X: X$ chromosome, $U$ : unknown location and $Y: Y$ chromosome). The length of elements is normalized by the length of consensus from full-length elements and represents the ages of each LTR retrotransposon. 


\section{Supplementary Table legend}

1444

1445

1446

1447

1448

1449

1450

1451

1452

1453

1454

1455

1456

1457

1458

1459

1460

1461

1462

1463

1464

1465

1466

1467

1468

1469

1470

1471

1472

1473

1474

1475

1476

1477

1478

1479

1480

1481

1482

1483

1484

1485

1486

1487
Table S1. The copy number of exons in conserved $\mathrm{Y}$-linked genes. We listed the copy number of each exon in conserved Y-linked genes based on BLAST results.

Table S2. The estimates of sensitivity and specificity of our $Y$-linked sequence assignment methods using $10-\mathrm{kb}$ regions with known chromosomal location. We calculated the median female-over-male coverage in our Illumina data in every 10-kb region with known chromosomal location. We then estimated the sensitivity and specificity of our methods using these data.

Table S3. Probe and primer information.

Table S4. The genomic location of duplicated exons in conserved $Y$-linked genes. We listed the genomic location of each exon in conserved $Y$-linked genes in our assemblies based on BLAST results.

Table S5. The intron length of all conserved $Y$-linked genes across species. We showed the length of each Y-linked exon in all conserved Y-linked genes based on BLAST results. If there are multiple copies of an exon, we choose the copy with a complete open reading frame and the highest expression level.

Table S6. Recent Y-linked duplications in $D$. melanogaster and species in the $D$. simulans clade. We list information on the recent $Y$-linked duplications and genes, including copy numbers, expression levels, phylogenies, and open reading frames. We also included some duplications from repetitive regions where we can date their origins.

Table S7. Enriched GO terms in Y-linked duplicated genes in D. melanogaster and the $D$. simulans clade. We searched the enriched $G O$ term from recently duplicated $Y$ linked genes from Table S6 using PANTHER (Released 20190711; [157]). We listed all GO terms significantly enriched in the duplication (FDR $<0.05$ ).

Table S8. The summary of conserved $Y$-linked genes and ampliconic genes expression. We summarized the expression level of conserved $Y$-linked genes and ampliconic genes. We sum up the gene expression for genes with multiple duplicated copies on $\mathrm{Y}$ chromosomes.

Table S9. The number of small RNA reads mapped to the repetitive sequences and $Y$-linked gene families in the $D$. simulans clade.

Table S10. Gene conversion rates for Y-linked ampliconic genes in the $D$. simulans clade. We listed the gene conversion rates and gene similarities on each $Y$ linked ampliconic gene family (e.g., Lhk-1, Lhk-2, and CK2ßtes-Y). We estimated gene conversion rates using both gene similarities $(p)$ and population recombination rates (Rmin and rho). 
Table S11. PAML results for branch and branch-site model analyses of $L$ h $k$ in the D. simulans clade. We showed raw results and LRT tests for branch and branch-site model analyses from PAML. We also report rates of protein evolution for each branch in each model and sites under positive selection in the branch-site model analyses.

Table S12. The number of new mutations observed in highly and lowly expressed copies of $Y$-linked gene families. We list the number of synonymous, nonsynonymous and UTR changes in highly and lowly expressed copies of $Y$-linked genes families. We suggest that highly expressed copies evolve under stronger selection (positive or purifying) than other copies. Therefore, we compared the number of synonymous changes over nonsynonymous changes in highly expressing copies to the other copies. See Table S21 for detailed information.

Table S13. PAML results for branch and branch-site model analyses of CK2ßtes-Y in the $D$. simulans clade. We showed raw results and LRT tests for branch and branch-site model analyses from PAML. We also report rates of protein evolution for each branch in each model and sites under positive selection in the branch-site model analyses.

Table S14. Indels in Y-linked duplications in D. melanogaster and the D. simulans clade. We listed the position and sizes of all indels we found in Y-linked duplications. We also inferred the potential microhomologies used for MHEJ repairing. We also infer other DSB repairing mechanisms, including tandem duplications and replication slippages, based on the sequence information.

Table S15. Polymorphic indels in $D$. melanogaster and $D$. simulans populations. We listed the position and sizes of polymorphic indels from $D$. melanogaster and $D$. simulans populations. We also inferred the potential microhomologies causing the deletions.

Table S16. The abundance of simple repeats in Illumina reads from male flies estimated with kseek and from our genome assemblies. We used kseek to measure the relative abundance of simple repeats in our Illumina reads. We also used TRF finder to calculate repeat contents in our assemblies. We compared the two results and picked probes for our FISH experiments.

Table S17. Repeat composition across chromosomes in D. melanogaster and the D. simulans clade. We list the composition of LTR retrotransposon, LINE, DNA transposons, satellite, simple repeats, rRNA, and other repeats across every chromosome in our assemblies.

Table S18. The detail of repetitive sequences across chromosomes in $D$. melanogaster and the $D$. simulans clade. We list the total sequence length from each transposon or complex repeat on $\mathrm{Y}$-linked contigs/scaffolds and other contigs/scaffolds in our assemblies. 
1534 Table S19. The Illumina coverage and blast result for each contig in the $D$.

1535 simulans clade. We used Blast v2.7.1+ [129] with blobtools (v1.0; [130]) to search the 1536 nt database (parameters "-task megablast -max_target_seqs 1 -max_hsps 1 -evalue 1e1537 25"). We estimated the lllumina coverage of each contig in males of $\bar{D}$. mauritiana, $D$. simulans and $D$. sechellia, respectively.

\section{Table S20. The summary of reads data used in this study} from each RNA-seq replicate and DNA-seq. We also recorded their frequency in our assembly and their translated amino acid. We estimated the expression level of each variant based on the SNP frequency in the genome. We also performed Welch's t-test to compare SNP frequency from DNA-seq and assemblies to it from RNA-seq. We further identify the SNPs associated with the allele that change more than 5 TPM compared to its estimated expression level from its frequency. The SNPs significant in the Welch's t-test and located in lowly or highly expressing alleles are chosen to perform the Chi-square test in Table S12. 